\title{
1 Kinetic principles underlying pioneer function of GAGA transcription factor in live cells \\ 2
}

3

4

5

2

\author{
Xiaona Tang ${ }^{1}$, Taibo $\mathrm{Li}^{1,2}$, Sheng Liu ${ }^{1}$, Jan Wisniewski ${ }^{3}$, Qinsi Zheng ${ }^{4}$, Yikang Rong ${ }^{5}$,
} Luke D. Lavis ${ }^{4}$, Carl Wu ${ }^{1,6, *}$

1. Department of Biology, Johns Hopkins University, Baltimore, USA

2. Department of Biomedical Engineering, Johns Hopkins University, Baltimore, USA

3. Experimental Immunology Branch, National Cancer Institute, Bethesda, USA

4. Janelia Research Campus, Howard Hughes Medical Institute, Ashburn, United States

5. State Key Laboratory of Bio-control, Institute of Entomology, School of Life Sciences, Sun Yat-sen University, Guangzhou, China

6. Department of Molecular Biology and Genetics, Johns Hopkins School of Medicine, Baltimore, United States

*Correspondence: wuc@jhu.edu

3

4

\section{Keywords: transcription/chromatin accessibility/pioneer factor/single-particle tracking/heat} shock 


\section{Abstract}

How pioneer factors interface with chromatin to promote accessibility for transcription control is poorly understood in vivo. Here, we directly visualize chromatin association by the prototypical GAGA pioneer factor (GAF) in live Drosophila hemocytes. Single-particle tracking reveals that the majority of GAF is chromatin-bound, with a stable-binding fraction showing nucleosome-like confinement residing on chromatin for over 2 minutes, far longer than the dynamic range of most transcription factors. These kinetic properties require the full complement of GAF's DNA-binding, multimerization and intrinsically disordered domains, and are autonomous from recruited chromatin remodelers NURF and PBAP, whose activities primarily benefit GAF's neighbors such as HSF. Evaluation of GAF kinetics together with its endogenous abundance indicates that despite on-off dynamics, GAF constitutively and fully occupies chromatin targets, thereby providing a temporal mechanism that sustains open chromatin for transcriptional responses to homeostatic, environmental, and developmental signals.

\section{Main}

Drosophila GAGA factor (GAF), a ubiquitous and essential Zn finger transcription factor (TF) encoded by the Trithorax-like (Trl) gene, is a multimeric protein complex that binds specifically to clusters of adjacent GAGAG sequences on numerous genes, including homeotic, steroid- and heat shock-response genes ${ }^{1-4}$. GAF regulates transcription by interactions with the TAF3 and TAF4 components of the TFIID general transcription factor ${ }^{5-7}$, the NELF elongation factor ${ }^{8}$, and antagonism to histone $\mathrm{H} 1$-mediated transcriptional repression ${ }^{9}$. In addition, as a pioneer transcription factor ${ }^{10,11}$, GAF is capable of binding to reconstituted nucleosomes ${ }^{12}$, directly recruiting chromatin remodelers NURF, PBAP and other factors ${ }^{6,13-15}$ to create accessible chromatin for neighboring non-pioneer factors ${ }^{16}$ and assembly of the paused RNA Polymerase II $(\text { Pol II })^{17,18}$. Beneficiaries of GAF pioneering activity in Drosophila include - heat shock factor HSF $^{19}$, coactivator $\mathrm{CBP}^{19,20}$, Polycomb repressor PHO (Pleiohomeotic) ${ }^{21}$, and the insulator binding complex LBC (Large Boundary Complex) ${ }^{22}$.

Genome-wide analysis reveals that GAF is enriched in vivo at promoters as well as distal cisregulatory regions comprising several thousand targets which often include clusters of tandem GA repeats ${ }^{17,23,24}$. GAF-specific RNA or protein depletion experiments have importantly demonstrated the in vivo function of GAF in generating chromatin accessibility in the Drosophila embryo and in cultured cells ${ }^{17,18,24,25}$. Despite these advances over decades of research, unifying principles for pioneering activity of TFs such as GAF have remained elusive. How pioneer factors differ from other sequence-specific transcription factors and the kinetic mechanisms by which pioneers perform their key genomic functions are subjects of continuing debate. 
To elucidate the underlying mechanisms of this pleiotropic transcription factor, we have studied the kinetics of GAF diffusion in the nucleoplasm and on genomic chromatin by singleparticle tracking (SPT) in live Drosophila hemocytes under different genetic contexts. In comparison, we also measured the kinetic parameters for HSF at normal and heat-stressed conditions. We then determined GAF and HSF protein levels in hemocytes, curated existing databases for numbers of genomic targets and integrated these parameters with the measured kinetics to obtain the target occupancy of each factor in vivo. Our findings uncover crucial quantitative principles for pioneering and maintaining chromatin accessibility over extended time periods even when the responsible factors continuously bind to and dissociate from their chromatin targets in a dynamic manner.

\section{Results}

\section{GAF imaging quantifies major chromatin binding fractions and prolonged residence times}

Drosophila $\mathrm{Trl}$ encodes two isoforms of GAF that harbor the $\mathrm{N}$-terminal POZ/BTB domain (hereafter called POZ), the central zinc-finger (ZF)-containing DNA binding domain (DBD), and long and short C-terminal Q-rich domains (Fig. 1A) ${ }^{4,26}$. We used CRISPR-Cas9-based gene editing to insert a HaloTag at the N-terminus of endogenous $\operatorname{Trl}$ (Fig. 1A and Fig. S1A-D). The Halo knock-in strain is homozygous-viable, expressing long and short Halo-GAF isoforms (GAF ${ }^{\mathrm{L}}$ and $\mathrm{GAF}^{S}$ ) as the sole source. Halo-GAF binds to numerous loci on salivary gland polytene chromosomes, consistent with immunostaining studies ${ }^{12,26,27}$ (Fig. 1B), and appears in multiple nuclear foci in diploid circulating larval hemocytes ( $>90 \%$ plasmatocytes, counterpart of mammalian macrophages ${ }^{28}$ ), similar to nuclear foci in the S2 cell line ${ }^{29}$ (Fig. 1C). We also constructed transgenic flies expressing C-terminal tagged isoforms $G A F^{\mathrm{L}}$-Halo and $\mathrm{GAF}^{\mathrm{S}}$-Halo, which exhibit tissue-specific expression ${ }^{26}$ (Fig. S1F) and are functionally active, as indicated by rescue of $\operatorname{Trl}^{13 C} / T r l^{R 67}$ lethal alleles ${ }^{3}$. Together, the data demonstrate that fusion of HaloTag does not interfere with the localization and essential functions of GAF.

We investigated the live-cell dynamics of tagged GAF species in live hemocytes (Fig. S2A) by SPT, using a 'fast-tracking' regime (10 ms/frame) in dSTORM mode ${ }^{30-32}$ to measure slow- and fastdiffusing molecules (Fig. 1D and Movie S1), and quantified diffusion coefficients with a robust, displacement-based, analytical protocol (Spot-On) ${ }^{33}$ (Fig. 1E and Fig. S2B). All Halo-tagged GAF versions display similar slow and fast diffusivities that are within similar dynamic range demonstrated by histone H2B (Fig. 1E and Fig. S2C), with $>75 \%$ and $>85 \%$ slow-diffusing fraction (chromatin-bound, $F_{\text {bound }}$ ), respectively. The diffusion coefficient (D) of the bound fraction $\left(D_{\text {bound }} 0.004-0.005 \mathrm{um}^{2} / \mathrm{s}\right.$ ) is two orders of magnitude lower than the free fraction $\left(D_{\text {free }} 0.75-0.8 \mathrm{um}^{2} / \mathrm{s}\right)$ (Fig. S2E-F).

A 'slow-tracking' regime (500 ms/frame) to motion-blur fast particles allowed selective detection of long- and short-lived chromatin-bound populations (Fig. S3A-C and Movie S2). We calculated 1-CDF of dwell times to generate survival curves demonstrating apparent GAF 
102

103

104

105

106

107

108

109

110

111

112

113

114

115

116

117

118

119

120

121

122

123

124

125

126

127

128

129

130

131

132

133

134

135

136

137

138

139

dissociation over time. Halo-GAF and $G A F^{L}$-Halo show similar profiles, with slightly faster decay for the $\mathrm{GAF}^{\mathrm{S}}$-Halo isoform (Fig. S3D). Fitting to a double exponential function (Fig. S3E) enabled calculation of long- and short-lived (called stable and transient) binding fractions, $f_{s b}$ and $f_{t b}$, and average stable and transient residence times, $\tau_{s b}$ and $\tau_{t b}$, after correction for photobleaching and out-of-focus chromatin motions using the apparent dissociation of Halo-H2B as an 'nondissociative' standard within the experimental timescale of several minutes (Fig. S3D). The stable binding fraction $\mathrm{f}_{\mathrm{sb}}$ multiplied by total binding fraction $\mathrm{F}_{\text {bound }}$ gives the overall stablebinding fraction $\mathrm{F}_{\mathrm{sb}}=30-35 \%$ (Fig. 1F and S2D). All stable-binding GAF fractions display strikingly protracted residence times $\tau_{\mathrm{sb}}$ : $130 \mathrm{~s}$ for Halo-GAF, $141 \mathrm{~s}$ for $\mathrm{GAF}^{\mathrm{L}}$-Halo, and $85 \mathrm{~s}$ for $\mathrm{GAF}^{\mathrm{S}}$-Halo, which is 20 - to 30 -fold longer than the transient residence time $\tau_{\text {tb }}$ of $\sim 4 \mathrm{~s}$ (Fig. 1G and Fig. S3F), and longer than $\tau_{s b}$ values measured for many mammalian transcription factors ${ }^{32,34-38}$. Stableand transient-binding are assumed to occur at cognate and non-specific sites, respectively.

\section{POZ, Q-rich, and DBD domains of GAF contribute to stable chromatin binding}

Purified, bacterially expressed GAF associates in oligomeric complexes ranging from monomers to multimers ${ }^{39,40}$, and GAF complexes are also observed in nuclear extracts of S2 cells. Multimerization is mediated by the POZ and Q-rich domains of $\mathrm{GAF}^{39-41}$. To investigate how GAF domains contribute to particle dynamics, we used CRISPR-Cas9 to engineer Halo-GAF deletions in the POZ domain $(\triangle P O Z)$, the zinc finger $\left(Z F^{9} ; Z F^{10}\right)$, and the long and short Q-rich domains $(\triangle Q)$ (Fig. 2A and Fig. S4A-E).

Homozygous $\triangle P O Z$ and $Z F^{9}$ or $Z F^{10}$ mutants arrest at early pupal and early $3^{\text {rd }}$ instar larval stages, respectively, while homozygous $\Delta Q$ is $70 \%$ viable, producing infertile adults with impaired longevity (Fig. 2A). These manifest phenotypes, although late in development owing to perdurance of the untagged WT GAF contributed by the heterozygous mother to homozygous oocytes, indicate that all three GAF domains are essential for Drosophila viability.

SPT using fast and slow tracking regimes for Halo-GAF $\triangle P O Z, Z F^{9}, Z F^{10}$ and $\triangle Q$ mutants in $3^{\text {rd }}$ instar larval hemocytes found that all three domain mutants display substantial reductions in $\mathrm{F}_{\mathrm{sb}}$ and $\tau_{\mathrm{sb}}$ for the slow-diffusing fraction (Fig. 2B-C and Fig. S5A-C). Disruption of the POZ domain reduces $F_{s b}$ from $29 \%$ to $15 \%$, and $\tau_{s b}$ from $130 \mathrm{~s}$ to $42 \mathrm{~s}$, demonstrating the important contribution of this multimerization domain to stable chromatin association. Deletion of Q-rich domains shows a modest reduction of $F_{s b}$ from $29 \%$ to $24 \%$, and reduces the $\tau_{s b}$ from $130 \mathrm{~s}$ to 49 s. Interestingly, under fast-tracking, $\triangle P O Z$ and $\triangle Q$ proteins exhibit similar $D_{\text {free }}$ but larger $D_{\text {bound }}$ compared to $W T$ GAF, suggesting a more diffusive binding mode (Fig. S5D-E). These results are consistent with the specific binding patterns of $\triangle P O Z$ and $\triangle Q$ on fixed polytene chromosomes (Fig. 2D)

The zinc finger mutants $Z F^{9}$ and $Z F^{10}$ also show reductions in $\mathrm{F}_{\mathrm{sb}}$ to $19 \%$ and $22 \%$, and $\tau_{\mathrm{sb}}$ to $\sim 40$ $S$ in hemocytes (Fig. $2 \mathrm{~B}-\mathrm{C}$ and Fig. S5A-C), similar to $\triangle P O Z$ and $\triangle Q$. However, unlike $\triangle P O Z$ and $\Delta Q$, both $Z F^{9}$ and $Z F^{10}$ mutants exhibit loss of the specific binding pattern on polytene chromosomes and increase of nucleoplasmic distribution (Fig. 2D). These results confirm the 
crucial function of the DNA-binding zinc finger for site-specific chromatin binding, and report aberrant, mechanistically unclear diffusive behavior of $Z F^{9}$ and $Z F^{10}$ mutant proteins. Notably, the time-averaged Mean Squared Displacement (MSD) curves from slow-tracking of $Z F^{9}$ and $Z F^{10}$ show an initial steep rise followed by a plateau after $10 \mathrm{~s}$, a profile dramatically different from $W T$ GAF, $\triangle P O Z, \triangle Q$, and $\mathrm{H} 2 \mathrm{~B}$ HaloTag fusions, all of which display a linear or Brownian increase of the MSD over a $60 \mathrm{~s}$ timescale (Fig. S5F).

\section{Chromatin binding kinetics by GAF is independent of recruited remodelers NURF and PBAP}

At cognate GAGAG sites on Drosophila chromatin, GAF recruits NURF and PBAP, ATP-dependent chromatin remodelers of the ISWI and SWI/SNF families, respectively, to drive DNA accessibility for neighboring transcription factors and establish promoter-proximal paused Pol II $^{13,17,19,24,42,43}$. This process begins during Drosophila embryogenesis, when GAF and the Zelda pioneer factor are individually required to activate and remodel the chromatin accessibility landscape for widespread zygotic transcription ${ }^{25,29}$. However, it was unclear whether the recruitment of NURF and PBAP by GAF is required to assist its own chromatin binding as a pioneer factor. To address this, we performed SPT of GAF - Halo and $\mathrm{GAF}^{\mathrm{S}}-\mathrm{Halo}$ on $3^{\text {rd }}$ instar larval hemocytes isolated from bap170 and nurf301/E(bx) mutants for unique subunits in PBAP $^{43,44}$ and NURF ${ }^{19,43}$ complexes, respectively (Fig. 3A). The results show little or small changes in the $F_{\text {bound, }}, D_{\text {bound }}$ and $\tau_{s b}$ values of the mutants (Fig. 3B-C and Fig. S6A-F). GAF ${ }^{L}$-Halo and GAF $^{S}$-Halo isoforms also show no qualitative global binding changes on polytene chromosomes in the bap170 and nurf301 mutants, although changes in a minority of chromosomal loci might escape detection (Fig. 3D). (By contrast, changes of HSF-Halo binding can be detected in mutants; see below). These findings are generally consistent with ChIP-Seq studies showing similar average GAF binding genome-wide in PBAP-depleted S2 cells (Fig. S7), although partial GAF binding is observed in a subset of Drosophila promoters (685 promoters displaying reductions in paused RNA Pol II and chromatin accessibility) ${ }^{24}$. Taken together, our live-cell SPT results indicate that GAF chromatin binding and dwell time are largely autonomous from NURF and PBAP, although other remodeling activities are not excluded ${ }^{45}$. We conclude that the main beneficiaries of NURF and PBAF recruitment and nucleosome remodeling activities are GAF's 'non-pioneer' neighbors.

\section{Heat shock increases chromatin-binding fraction of HSF without affecting dwell time}

The recruitment of chromatin remodelers by GAF increases accessibility to facilitate binding of Heat Shock Factor (HSF) to the tripartite Heat Shock Element (HSE) adjacent to GAF binding sites at heat shock promoters ${ }^{18,46-49}$. Under normal conditions, HSF is predominantly monomeric, with low (submicromolar) affinity of its winged-helix DBD for an NGAAN sequence ${ }^{50,51}$; heat shock induces HSF trimerization and juxtaposition of three DBDs for highaffinity binding to HSEs containing triple NGAAN sequences in alternating orientation ${ }^{52-57}$.

To measure HSF dynamics, we constructed a transgenic HSF-Halo strain under natural expression control, and verified the functionality of HSF-Halo by rescue of $P\{P Z\} H s f^{03091} / H s f^{3}$ lethal alleles ${ }^{58,59}$. We further validated HSF-Halo functions by confocal imaging of fixed polytene 
nuclei, which showed that HSF-Halo is mostly nucleoplasmic at room temperature (RT), except for low binding to few sites including Hsp83 harboring very high affinity HSEs; heat shock at $37.5{ }^{\circ} \mathrm{C}$ for 10 or $30 \mathrm{~min}$ induced strong HSF binding to many more chromosomal loci, most prominently reported at $\mathrm{Hsp}$ genes ${ }^{52,60}$ (Fig. S8A). This inducible pattern of HSF binding on heat shock is partially reduced in mutants for Trl, Bap170 and Nurf301 (Fig. S8A). (Note that there are GAF-independent HSF targets in the genome ${ }^{18}$, for which changes of HSF binding at corresponding polytene loci would not be expected). Imaging of fixed hemocytes shows that the heterogeneous distribution of HSF-Halo changes on heat shock to a more punctate pattern including several prominent foci, consistent with previous studies (Fig. 4A) (2,61,62. $^{\text {. }}$

We performed live-cell SPT on HSF-Halo in fast- and slow-tracking modes in the $P\{P Z\} H s f^{03091} / H s f^{3}$ genetic background, using hemocytes cultured at RT or heat shocked at $37.5{ }^{\circ} \mathrm{C}$ (Fig. 4B). As expected, the overall binding $\mathrm{F}_{\text {bound }}$ of HSF from fast-tracking increases substantially from $24.9 \%$ to $44.3 \%$ upon heat shock (Fig. 4 C and Fig. S8B). Importantly, twocomponent exponential decay fitting of the HSF-Halo survival curves derived from slow tracking reveals a substantial increase of stable binding $\mathrm{F}_{\mathrm{sb}}$ from $5.9 \%$ to $14.2 \%$ upon heat shock with no measurable change of residence time $\tau_{\mathrm{sb}}(47 \mathrm{~s})$ (Fig. 4C-D and Fig. S8C, E). Thus, heat shock elevates the stable chromatin-binding HSF trimer fraction without affecting the dissociation rate (inverse of residence time), and suggests that the limited stable binding at $R T\left(F_{s b}=5.9 \%\right)$ is due to low-level trimerization ${ }^{57}$. Distinct from HSF dynamics, GAF shows a small overall reduction in $\mathrm{F}_{\text {bound }}$ on heat shock (from $77.3 \%$ to $69.6 \%$ ), and similarly for the stable binding fraction $\mathrm{F}_{\mathrm{sb}}$ (from $29.4 \%$ to $22.1 \%$ ) (Fig. 4C). Importantly, the residence time $\tau_{\mathrm{sb}}$ for GAF remains unchanged after heat shock (Fig. 4D and S8D-E).

\section{Chromatin-bound GAF displays H2B-like confinement}

The diffusion coefficient of chromatin-bound HSF measured by fast-tracking ( $D_{\text {bound, }}$, average of both stable- and transient-binding) at RT where HSF monomers predominate is $\sim 4$-fold greater than that of HSF trimers induced by heat shock ( $D_{\text {bound }}=0.075$ vs $0.019 \mathrm{um}^{2} / \mathrm{s}$ ) (Fig. 4E). HSF monomers also exhibit $>10$-fold larger $D_{\text {bound }}$ values than $G A F\left(D_{\text {bound }}=0.0046 \mathrm{um}^{2} / \mathrm{s}\right)($ Fig. $4 E)$, indicating that a single DBD is more diffusive on chromatin than multiple DBDs. Intriguingly, $D_{\text {bound }}$ for GAF approaches the $\mathrm{H} 2 \mathrm{~B}$ value $\left(D_{\text {bound }}=0.0020 \mathrm{um}^{2} / \mathrm{s}\right)$ (Fig. $4 E$ ). To strengthen these findings, we analyzed particle trajectories with vbSPT, a variational Bayesian Hidden Markov Model (HMM) algorithm that assigns bound and free diffusive states to individual particle displacements of each trajectory ${ }^{63,64}$ (Fig. S9A-B). We classified particle trajectories as either 'bound' or 'free', excluding a small fraction showing two-state diffusivity (Fig. S9A-D). The timeaveraged MSD plots of the bound particles confirm that bound molecules move in small confined regions (Fig. 4F and Fig. S10A), while free molecules undergo Brownian motion (Fig. S10B). The MSD plot of chromatin-bound GAF trajectories reaches a plateau at low values, resembling that of $\mathrm{H} 2 \mathrm{~B}$ (Fig. S10A), while HSF plateaus at higher values (Fig. 4F). The radius of confinement $\left(R_{C}\right)$ gives median values for HSF monomers $(0.13 \mu \mathrm{m})$, HSF trimers $(0.10 \mu \mathrm{m})$, GAF multimers $(0.07 \mu \mathrm{m})$, and $\mathrm{H} 2 \mathrm{~B}(0.06 \mu \mathrm{m})$ (Fig. S10C). Together, the results indicate that 
217 chromatin-bound GAF is nearly as constrained as nucleosomal histones. Activated HSF trimers

218 are less constrained than GAF, possibly due to fewer DBDs per complex and/or higher local

219 chromatin mobility, but bound HSF monomers are more diffusive, consistent with the presence

220 of only a single DBD.

221 Constitutively high temporal occupancy defines pioneering activity

222 The steady-state open chromatin landscape at promoters and enhancers featuring nucleosome-

223 depleted regions genome-wide ${ }^{45,65,66}$ belies highly dynamic interactions with transcription and 224 chromatin factors ${ }^{67-72}$. The establishment and maintenance of chromatin accessibility, i.e. the

225 sustained opening of chromatin, requires the joint activities of sequence-specific DNA-binding

226 factors and ATP-dependent remodeling enzymes ${ }^{12,19,24,25,73,74}$, the latter proteins interacting

227 with chromatin with a lifetime of seconds ${ }^{67,69,70}$. GAF directs pioneering functions not only by

228 virtue of its affinity for nucleosomal DNA targets ${ }^{12,75}$ but also recruitment of chromatin

229 remodelers ${ }^{12,14,15,24,42,76}$. Given the highly transient association and variable occupancy levels

230 displayed by remodelers ${ }^{67,70}$, we hypothesized that GAF should instead sustain high occupancy

231 along with protracted dwell time to continuously maintain open chromatin at cognate targets .

232 Temporal occupancy, the percent time of any duration for which a cognate site is factor-bound, 233 depends on the number of GAF molecules ( $\left.N_{\text {molecules }}\right)$ per cell and the number of target sites $234\left(\mathrm{~N}_{\text {sites }}\right)$ in the genome. In the context of the facilitated diffusion model ${ }^{77}$ in which transcription 235 factors experience 3D nucleoplasmic diffusion, nonspecific binding, 1D diffusion, dissociation 236 and re-binding until site-specific chromatin engagement, temporal occupancy is also dependent 237 on the kinetics of target search and dissociation. Integration of our kinetic data from SPT with published genomic data allows calculation of temporal occupancy for GAF.

239

ChIP-seq identifies 3622 high-confidence GAF peaks from the hemocyte-like S2 cell line ${ }^{17,78,79}$.

240 Similar numbers of GAF peaks are found by ChIP-seq analysis of larval imaginal tissues and embryos although the peaks from different cell types overlap partially ${ }^{25,80}$. We measured GAF abundance by fluorescence flow cytometry using a calibrated CTCF standard $^{81}$, estimating $\mathrm{N}_{\text {molecules }}=56,683 \pm 6,025 \mathrm{GAF}$ molecules per hemocyte (Fig. S11A). Given that circulating hemocytes are largely in the G2 cell cycle phase (Fig. S11B-C), we estimate $\mathrm{N}_{\text {sites }}=3622 \times 4$ genome copies $=14,488$. From the overall chromatin-binding fraction $\left[F_{s b}\right]$, residence times and fractions for stable and transient chromatin-binding $\left(\tau_{s b}, f_{s b}, \tau_{t b}, f_{t b}\right)$, we derived the average search time $\left(\tau_{\text {search }}=150 \mathrm{~s}\right.$, the time from GAF dissociation from one stable target to association with the next) and the sampling interval ( $S I=71.5 \mathrm{~s}$, the time from the start of one stablebinding event to the next stable event on the same chromatin target; see methods), assuming that GAF binds stably at specific sites and transiently elsewhere (Fig 5A-B). nonshock conditions and at $154 \%$ after heat shock, assuming no change in the number of GAF molecules and targets. The occupancy for $\Delta Q\left(N_{\text {molecules }}=66,206 \pm 3,064\right)$ remains similar at $177 \%$ and is reduced to $89 \%$ for $\triangle P O Z$ ( $\left.N_{\text {molecules }}=44,907 \pm 14,154\right)$ (Fig. 5B). The 182\% occupancy for 
WT GAF is averaged over the 3622 GAF peaks whose intensities are correlated with increasing numbers of GAGAG elements. Notably, $\sim 65 \%$ of GAF peaks harbor more than two nonoverlapping GAGAG elements, with median peak intensity rising to a plateau at 6-7 clustered elements ${ }^{17}$ (Fig. 5C), e.g. at ubx, engrailed, E74, eve, and Hsp genes ${ }^{4}$. The results indicate that GAF binds at least as a dimer on average, with a distribution tending towards larger oligomers for peaks showing high ChIP-seq signals, which can be attributed to the cooperative binding of $\mathrm{GAF}$, as demonstrated in vitro ${ }^{39,40}$. At this subset of highly-enriched sites, GAF may bind as a multimeric complex with essentially full temporal occupancy despite factor on-off dynamics (Fig. 6) for a time period of any duration in which GAF levels and the number of GAF targets remain unchanged.

We note that many GAF-binding peaks overlap with Pipsqueak, a related POZ-domain transcription factor ${ }^{82,83}$ and partially with CLAMP, another GA repeat-binding pioneer factor in Drosophila $^{84,85}$. The overall site occupancy at GAF locations on chromatin is therefore likely to be further supplemented when the contributions of Pipsqueak and CLAMP are quantified.

For HSF, we determined $N_{\text {molecules }}=9,543 \pm 613$ for a sole source, transgenic HSF-Halo under natural expression control in the $P\{P Z\} H s f^{03091} / H_{s f}^{3}$ background (Fig. S11A). A similar calculation for HSF-Halo binding to 442 genomic sites after heat shock $^{48}$ gives $\tau_{\text {search }}=119 \mathrm{~s}$, and SI $=31 \mathrm{~s}$, which results in an average HSF occupancy of $153 \%$, or $51 \%$ for HSF trimers as the predominant species induced after 10-40 min heat shock (Fig 5B). At highly-enriched HSF locations such as the major Hsp genes harboring several HSEs, it follows that one or more HSF trimers may occupy the promoter $100 \%$ of the time on full induction to release the paused RNA Pol II and rapidly recruit additional enzymes for a burst of transcription until system attenuation (Fig. 6).

\section{Discussion}

\section{Full complement of GAF domains promotes kinetics of stable chromatin association}

Our single-particle tracking of GAF in live hemocytes reveals that GAF binds chromatin with an exceptionally long residence time $\tau_{s b}$ of $\sim 2 \mathrm{~min}$ compared to other factors. Systematic mutagenesis showing that the DBD, the POZ multimerization domain and the Q-rich domains are required for viability also found that not just the DNA-binding Zinc finger, but all three GAF domains are required for its stable chromatin association and long $\tau_{\mathrm{sb}}$ (Fig. 2B-C). Of interest, the Zinc finger mutants $Z F^{9}$ and $Z F^{10}$ have a substantial residual slow-diffusing fraction in hemocytes, but show no specific binding on polytene chromosome bands (Fig. 2B, D). This is possibly due to non-specific association of the altered zinc finger and the remaining basic regions of the DBD (Fig. 2A and Fig. S4), and/or protein-protein interactions of the intact POZ or Q-rich IDR domains (intrinsically disordered regions). Overall, our findings are consistent with the contributions to specific and non-specific DNA binding shown by mutant DBDs of mammalian TFs ${ }^{32,36,86}$, and with the observation of IDR-assisted, in vivo DNA binding specificity for two yeast TFs ${ }^{87}$. Of note, mutant GAF proteins $Z F^{9}$ and $Z F^{10}$ also exhibit an aberrant, slow- 
diffusing species whose underlying mechanism is unknown and remains to be further explored (Fig. S5F).

The POZ domain is found at the $\mathrm{N}$-terminus of vertebrate and invertebrate transcriptional regulators implicated in development and disease $\mathrm{e}^{88}$. Functionally, the POZ domain is involved in protein homo- and hetero-dimerization, as well as multimerization ${ }^{88}$. The POZ domain mediates multimerization of GAF, which facilitates cooperative binding to closely clustered GAGAG elements $^{39,40}$, and assists long-distance promoter-enhancer interactions between wellseparated GAGAG clusters ${ }^{89}$. As judged by the kinetic behaviors of the GAF POZ mutant, we conclude that multimerization of GAF constitutes a critical element for its ability to pioneer open chromatin. Similarly, a variant glucocorticoid receptor $(G R)$ that mimics allosterically induced GR tetramerization converts GR to a super-receptor that enhances chromatin occupancy at normally inaccessible sites ${ }^{90}$.

\section{Autonomy from recruited chromatin remodelers}

The coupling of GAF-mediated pioneering of chromatin accessibility to ATP-dependent chromatin remodeling activities has been reported from the outset of studies on the mechanism underlying DNase hypersensitive sites ${ }^{12}$. Biochemical experiments have since demonstrated that GAF directly recruits remodelers NURF and PBAP via protein-protein interactions, in addition to a number of other chromatin-based factors ${ }^{76}$. Using mutants for NURF and PBAP, we now show that their recruitment is not obligatory for GAF to kinetically engage chromatin targets. This indicates that GAF is largely autonomous from recruited remodelers and that the ensuing chromatin remodeling to antagonize competing processes of nucleosome encroachment primarily benefits the binding or activity of neighboring TFs to chromatin targets. While other factors or the global background of remodeling activities are not excluded from modulating GAF binding, GAF's relative autonomy from two prominent recruited members of the SWI/SNF and ISWI remodeler families at the initial step of chromatin association may define an important property of transcription factors that act as pioneering agents.

\section{High constitutive and ind ucible temporal occupancies by GAF and HSF}

By curating genomic databases for the number of genomic targets and integrating these parameters with measured kinetics and abundance, we found that GAF binds to its target sites with temporal occupancy of $182 \%$ for 3622 high-confidence ChIP-seq peaks ${ }^{17}$ (Fig. 5A-C), i.e. with near full occupancy as a dimer on average. For genomic sites with greater than average GAF ChIP enrichment and number of GAGAG elements (Fig. 5B), this occupancy is likely to involve higher oligomers, consistent with the native biochemical states of GAF complexes. Such high occupancy, or kinetic persistence, whereby factor dissociation from chromatin and replacement are essentially simultaneous, maintains a constant barrier and magnet for remodeler recruitment at GAF targets. (GAF multimers appear as stable biochemical complexes $^{39,40}$, but dynamic exchange of GAF monomers within a multimeric complex is 
possible in principle and awaits further study). We envision that a substantial fraction of GAF targets in the genome displays high oligomeric status and temporal occupancy ( $100 \%)$, while the remaining targets showing progressively lower occupancies, consistent with the genomewide continua of transcription factor binding levels on metazoan genomes that reflect functional, quasi-functional, and nonfunctional transcription control ${ }^{91,92}$.

Unlike GAF, HSF monomers under room temperature conditions inducibly trimerize on heat shock $^{57}$ which significantly increases the chromatin-bound fraction $\left(F_{\text {bound }}\right)$ without changing stable residence time $\left(\tau_{\mathrm{sb}}\right)$. For $\sim 400$ activated HSF-binding sites on the Drosophila genome ${ }^{48}$, we estimate an average of $\sim 50 \%$ temporal occupancy by one HSF trimer. At the major Hsp loci harboring greater than average ChIP-seq signal intensity and multiple HSE elements, we envision that one or more HSF trimers engage at near full (100\%) temporal occupancy. Full occupancy by HSF on heat shock induction may be required to facilitate release of the paused RNA Polymerase II previously established by GAF, and to sustain recruitment-release of new transcription pre-initiation complexes for a strong transcriptional burst of HS-responsive genes.

\section{Pioneering of chromatin accessibility is a process involving multiple inputs}

The pioneer transcription factor concept has been introduced and elaborated for over two decades with a focus on special nucleosome binding properties of the FoxA1 prototype proposed to initiate establishment of chromatin accessibility for the benefit of consequent binding of neighboring TFs ${ }^{93}$. However, there continues to be debate whether the reported properties of FoxA1 are sufficiently distinct to set it apart from other transcription factors ${ }^{16,35,94,95}$. Our early findings on GAF that predate the controversy have documented that nucleosome binding and ATP-dependent remodeling are functionally coupled in a biochemical assay $^{12}$, and additional studies to the present - including the genome-wide effects of remodeler depletion on chromatin accessibility ${ }^{24}$ and nucleosome positioning at GAF targets ${ }^{73}$ - support the concept that GAF directly recruits remodelers for the site-specific creation of accessible chromatin.

Thus, there is ample evidence to include remodeler recruitment by GAF or other $\mathrm{TFs}^{35}$ as a fundamental biochemical criterion for pioneering besides affinity for nucleosomes or closed chromatin $^{36}$. Our finding of autonomous remodeler recruitment (chromatin interaction kinetics of GAF being largely unaffected in NURF and PBAP mutants) provides additional insight on the hierarchical nature of pioneering wherein GAF binding to chromatin at the initial stage of pioneering (stage 1) would be followed by remodeler recruitment and ATP-dependent nucleosome mobilization to create DNase hypersensitivity, thereby facilitating assembly of the transcription preinitiation complex (PIC), paused Pol II, and the inducible binding of HSF (stage 2) (Fig. 6). In addition to remodeler recruitment, constitutively full temporal occupancy by GAF revealed by the single-particle kinetics provides a quantitative criterion for pioneering longterm chromatin accessibility primed for the transcriptional responses to homeostatic, environmental, and developmental signals. 
However, we emphasize that high temporal occupancy is not an obligatory consequence of GAF's long residence time on chromatin, or its multimeric, cooperative binding to GAGAG elements. Occupancy is also dependent on cellular GAF expression, abundance, and the number and genomic distribution of GAGAG elements in Drosophila. Thus, it may be instructive to consider pioneering as an active process with the multiple inputs we have described autonomous factor-binding to closed chromatin, remodeler recruitment, nucleosome mobilization, and for the subset of transcription factors that maintain accessible chromatin constitutively, correspondingly high temporal occupancy, not excluding additional criteria to be identified. We hope that inclusion of these biochemical and kinetic principles guides further investigations on the substantial fraction of computationally identified human TFs ( $16 \%$ of $~ 700$ $\mathrm{TFs})^{16}$ that may pioneer chromatin accessibility as a basic mechanism of genome regulation in eukaryotic organisms.

\section{Methods}

\section{Fly strain construction}

\section{CRISPR/Cas9-mediated genome editing}

HaloTag was inserted downstream of the start codon of endogenous $T r l$ via homology-directed repair (HDR) and CRISPR/Cas9 to generate the Halo-GAF knock-in strain (Fig. S1 A-C). The donor repair template was constructed on the pScarlessHD-DsRed plasmid (a gift from Kate O'Connor-Giles, Addgene plasmid \# 64703), which contains a DsRed selection marker cassette flanked by PBac transposon ends and TTAA sites ${ }^{97}$. The donor plasmid was designed such that after HDR, the DsRed cassette is inserted into a nearby genomic TTAA site adjacent to the gRNA target in the coding region that is close to the ATG start codon. Approximately $1 \mathrm{~kb}$ downstream of the gRNA site was cloned as the right homology arm (RHA), with silent mutations introduced to destroy the gRNA sequence in the donor plasmid. Similarly, $1 \mathrm{~kb}$ upstream of the genomic TTAA site was cloned as the left homology arm (LHA). LHA and RHA mediate HDR upon Cas9 cleavage, inserting HaloTag along with the DsRed cassette. Flies that underwent HDR were identified with DsRed eye fluorescence. The DsRed cassette was removed with a single cross to a fly strain expressing PBac transposase, as indicated by loss of fluorescence, leaving only one TTAA site, thus allowing scarless HaloTag knock-in with a removable selection marker. A flexible linker GGSGS was added between Halo and GAF. The HaloTag knock-in was verified by fluorescent staining and DNA sequencing.

After constructing the Halo-GAF strain, deletions of the Halo-GAF fusion protein were generated by CRISPR-Cas9 gene editing. A 90 bp (30 AA, $\triangle 90-119$ ) precise deletion was generated in the POZ domain $(\triangle P O Z)$ by HDR. Small deletions in the zinc finger of the DBD $\left(Z F^{9}\right.$, $\mathrm{R} 356 \Delta, \mathrm{N} 357 \Delta$; and $Z F^{10}, \mathrm{R} 356 \Delta$ ) were generated by random indels. For $\triangle Q$, two gRNAs 
407

408

409

410

411

412

413

414

415

416

417

418

419

420

421

422

423

424

425

426

427

428

429

430

431

432

433

434

435

436

437

438

targeting the Q-rich domains of the long or short GAF isoforms were introduced at the same time to screen for indels creating frameshifts and truncations of both Q-rich domains. To screen for desired mutants, lethal or reduced viability strains were selected and characterized by PCR and DNAsequencing.

All gRNAs were cloned into pCFD5. Donor and gRNA plasmids were mixed to a final concentration of $200 \mathrm{ng} / \mathrm{uL}$ and $500^{\sim} 600 \mathrm{ng} / \mathrm{uL}$, respectively, and injected into fly strains expressing Cas9 in the germline (yw;nos-Cas9(II-attP40), a gift from NIG-FLY, Japan). To generate Halo-GAF mutants, the Halo-GAF knock-in strain was crossed to yw;nos-Cas9(II-attP40) for injection. All fly embryo injections were performed by BestGene Inc (CA).

\section{Transgenic fly construction via Phic31 integrase}

Trl gene and $\sim 1 \mathrm{~kb}$ flanking genomic sequence was cloned from a BAC genomic clone (BACR11B23) into pattB (backbone taken from pattB-aubergine-ADH-gf, Addgene plasmid \# 69448, a gift from Phillip Zamore) via recombineering ${ }^{98}$. HaloTag was inserted upstream of the stop codon for the long and short isoforms, respectively, along with a removable Cam ${ }^{R}$ selection cassette via recombineering ${ }^{99}$. The CamR cassette was flanked by the 8-bp Notl restriction sites (plus an additional bp to ensure that HaloTag is in frame) and removed by Notl digestion and re-ligation, leaving a GGSGSAAA linker sequence between GAF and HaloTag. The constructs were incorporated into the attP2 site in the Drosophila genome via PhiC31 integrase $^{100}$ (Fig. S1E), generating the $\mathrm{GAF}^{\mathrm{L}}$-Halo and $\mathrm{GAF}^{\mathrm{S}}$-Halo transgenic strains, which express a Halo-tagged long or short GAF isoform and the other untagged isoform. The functionality of recombinant fusion proteins was verified by rescue of the lethal alleles $T r 1^{13 C} / T r l^{R 673}$. We similarly generated the HSF-Halo transgenic fly strain at the attP2 site from the genomic clone BACR33K09. The functionality of HSF-Halo was verified by rescue of $P\{P Z\} H s f^{03091} / \mathrm{Hsf}^{3}$ lethal alleles ${ }^{58,59}$. The Halo-H2B transgenic strain was similarly constructed at the attP2 site, with a $\sim 4.9 \mathrm{~kb}$ DNA fragment containing five Drosophila histone genes for HaloTag insertion at the $\mathrm{N}$-terminus of H2B.

\section{Mutant fly strains}

Mutant alleles were obtained from the Bloomington Drosophila Stock center (BDSD, IN): $\operatorname{Tr}^{13 C}$ (BDSC:58473); $\operatorname{Tr}^{R 67}$ (BDSC:58475); $P\{P Z\} H s f^{03091}$ (BDSC:11271); Hsf ${ }^{3}$ (BDSC:5488); P\{EP\}Bap170 ${ }^{65986}$ (BDSC:28471); Bap170 ${ }^{1135}$ (BDSC:63807); Nurf3014 (BDSC:9904); and Df(3L)Exel6084 (BDSC:7563).

Genotypes of fly strains in Fig. 3 and Fig. S6 (only GAF ${ }^{L}$-Halo isoform is shown, GAF ${ }^{\mathrm{s}}$-Halo strains have the same corresponding genotypes except expressing $\mathrm{GAF}^{\mathrm{s}}$-Halo):

WT: $p\left\{G A F^{L}\right.$-Halo\}attP2

bap170: Bap170 $135 / P\{E P\} B a p 170^{G 5986} ; p\left\{G A F^{L}\right.$-Halo\}attP2 (generated by crossing Bap170 ${ }^{135} ; p\left\{G A F^{L}\right.$-Halo\}attP2/T(2;3)TSTL, CyO: TM6B, Tb $^{1}$ to $P\{E P\} B a p 170^{G 5986} ; p\left\{G A F^{L}\right.$ Halo\}attP2/ T(2;3)TSTL, CYO: TM6B, $T b^{1}$ ) 
nurf301: Nurf301 ${ }^{4}, p\left\{G A F^{L}-H a l o\right\} a t t P 2 / D f(3 L) E x e l 6084, p\left\{G A F^{L}\right.$-Halo\}attP2 (generated by crossing Nurf301 ${ }^{4}, p\left\{G A F^{L}\right.$-Halo\}attP2/TM6B, $T b^{1}$ to $D f(3 L) E x e l 6084, p\left\{G A F^{L}\right.$ Halo\}attP2/TM6B, $T b^{1}$ )

Genotypes of fly strains in Fig. S8A:

WT: $p\{$ HSF-Halo\}attP2

trl: $\operatorname{Tr}^{13 C}, p\left\{\right.$ HSF-Halo\}attP2/TrIR ${ }^{67}, p\left\{\right.$ HSF-Halo\}attP2 (generated by crossing $\operatorname{Trl}^{13 C}$, p $\left\{\right.$ HSF-Halo\}attP2/TM6B, $T b^{1}$ to $\operatorname{Trl}^{67}$, p $\left\{\right.$ HSF-Halo\}attP2/TM6B, $T b^{1}$ ) bap170: Bap170 $135 / P\{E P\} B a p 170^{G 5986} ; p\{$ HSF-Halo\}attP2 (generated by crossing

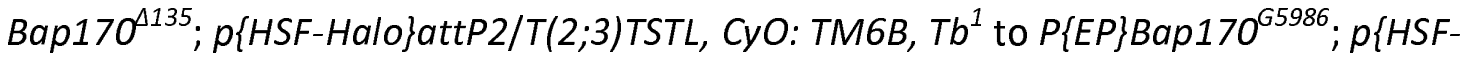
Halo\}attP2/ T(2;3)TSTL, CyO: TM6B, $T b^{1}$ )

nurf301: Nurf301 ${ }^{4}$, p $\{$ HSF-Halo\}attP2/Df(3L)Exel6084, p $\{$ HSF-Halo\}attP2 (generated by crossing Nurf301 ${ }^{4}, p\{H S F-H a l o\} a t t P 2 / T M 6 B, T b^{1}$ to Df(3L)Exel6084, $p\{H S F-$ Halo\}attP2/TM6B, $T b^{1}$ )

\section{Single-particle imaging in live Drosophila hemocytes}

\section{Sample preparation}

Single-molecule live cell imaging was performed with $3^{\text {rd }}$ instar larval hemocytes, representing mainly plasmatocytes ( $>90 \%$ of Drosophila hemocytes). Hemocytes were released from 5-10 thoroughly washed larvae into a sample chamber containing $1 \mathrm{~mL}$ filtered Schneider's Drosophila medium (Gibco ${ }^{\mathrm{TM}} 21720024$ ) including an EDTA-free protease inhibitor cocktail (Roche 4693159001). The sample chamber is an Attofluor ${ }^{\mathrm{TM}}$ Cell Chamber (Invitrogen, A7816) assembled with round coverglass (Electron Microscopy Sciences, 72290-12) cleaned by flaming. Cells were stained with $0.2^{\sim} 1.5 \mathrm{nM}$ JF552/JFX554 for $30 \mathrm{~min}$ at room temperature, during which hemocytes adhered to the coverglass bottom of the imaging chamber. During incubation steps, sample chambers were covered with aluminum foil to minimize evaporation and block light. Cells were then briefly washed twice with Schneider's media without protease inhibitor and imaged on a custom-built wide-field SPT fluorescence microscope ${ }^{101}$.

\section{Single-particle imaging}

All single-particle imaging were carried out on an Axio Observer Z1 microscope (Zeiss, Germany) equipped with an Plan-Apochromat 150x/1.35 glycerin-immersion objective (ZEISS, Germany), and a C9100-13 EM-CCD camera (Hamamatsu Photonics, Japan) featuring 512x512 pixels with $16 \mu \mathrm{m}$ pixel size. The pixel size of recorded images is $16 \mu \mathrm{m} / 150=107 \mathrm{~nm}$. JF552/JFX554 were excited with a CL555-100 $555 \mathrm{~nm}$ laser (CrystaLaser, Reno, NV) and the emission light was passed through a filter cube containing a $561 \mathrm{~nm}$ BrightLine single-edge beamsplitter, a 612/69 $\mathrm{nm}$ BrightLine single-band bandpass emission filter (Semrock, Rochester, NY), then through a $750 \mathrm{~nm}$ blocking edge BrightLine multiphoton short-pass emission filter and a 405/488/561/635 $\mathrm{nm}$ StopLine quad-notch filter (Semrock, Rochester, NY) before entering the camera. The EM- 
CCD camera was operated at $\sim-80^{\circ} \mathrm{C}$ (forced-air cooling) and $1200 x$ EM gain. We used the ZEN (ZEISS, Germany) and HCImage (Hamamatsu Photonics, Japan) software to operate the microscope and camera, respectively. Imaging was carried out at room temperature except for heat shock experiments in which samples were imaged in a stage-top incubator with temperature control (H301-MINI chamber with UNO-T-H controller, Okolab, Italy)

In the fast-tracking regime, time-lapse movies with a $128 \times 128$ pixel field of view were acquired with high laser power ( 1 kW/ $\left.\mathrm{cm}^{2}\right)$ and $10 \mathrm{~ms}$ exposure time for 1.5-2 min. Initial laser excitation leads to simultaneous emissions of all labeled molecules, marking locations of individual nuclei in the field of view. Cells with relatively homogenous initial nuclear glow (interphase) were imaged. Emitting molecules quickly enter the "dark" state and stochastically reemit. Cells were stained with JFX554, with the concentration optimized (1-1.5 nM) to achieve sparse single-particles per nucleus per frame after $10^{\sim 30} \mathrm{~s}$ of the initial glow, minimizing mistracking.

For slow tracking, we used low laser power $\left(\sim 36 \mathrm{~W} / \mathrm{cm}^{2}\right)$ and imaged a $256 \times 256$ pixel field of view with $500 \mathrm{~ms}$ exposure time for 2-5 min. We labeled cells with $50 \mathrm{nM}$ of a far-red dye JF700 to block most Halo-tagged proteins and at the same time adjusted a low concentration of JF552 to visualize only 2-10 molecules/frame. This sparse labeling approach allows tracking of chromatin-bound molecules with minimal photobleaching.

\section{Single-particle data processing and statistical analysis}

\section{Image pre-processing}

Raw time-lapse data were pre-processed in $\mathrm{Fiji}^{102}$ to convert to 16 -bit TIFF format and extract a substack with sparse single particles ( $<5$ particles/nucleus for fast tracking, and $<10$ particles/nucleus for slow tracking). A maximum-intensity Z projection of each movie was generated to outline cell nuclei as the ROI. A corresponding binary mask was created to isolate nuclear trajectories for subsequent analysis.

\section{Single-particle localizing and tracking}

Single particles were localized and tracked using the open-source program DiaTrack v3.05 ${ }^{103}$. Tracking was performed with a 6 pixel $(\sim 0.65 \mu \mathrm{m})$ maximum jump allowance between consecutive frames for fast tracking. All factors we imaged showed displacements within this cut-off as informed by the frequency histogram (Fig. S2B, S5A, S6A, and S7B). Since slow tracking selectively imaged bound particles, the maximum jump allowance between consecutive frames was set as 3 pixels ( $0.32 \mu \mathrm{m})$ to minimize misconnection. HSF-Halo showed displacement histograms with a smooth tail within this range, while Halo-GAF and Halo-H2B displacements were mostly within $0.2 \mu \mathrm{m}$ (not shown). For analysis of slow-tracking experiments to measure residence times, we allowed gaps in trajectories to account for blinking or missed localizations, with 3-frame maximum blinking and a more stringent 2-pixel maximum jump. 


\section{Analyzing fast-tracking data}

518 We used a custom R package Sojourner (https://github.com/sheng-liu/sojourner) to extract

519 trajectories from MATLAB files generated by DiaTrack, which contain information on $\mathrm{x}, \mathrm{y}$

520 coordinates and frame number (time) that were applied for computation of kinetic parameters.

521 Trajectories found within the nucleus were isolated ('masked' trajectories) using Sojourner and

522 binary masks generated during pre-processing. Average length of trajectories was 11-21

523 displacements (median $=5-8$ ).

524 Spot-On

525 We used Spot-on to perform two-state kinetic modeling of displacements from all 'masked'

526 trajectories ${ }^{33}$, to derive diffusion coefficients $\left(D_{\text {bound }}, D_{\text {free }}\right)$ and the corresponding fractions

527 ( $\left.F_{\text {bound }}, F_{\text {free }}=1-F_{\text {bound }}\right)$. The Spot-On python package was used with the following parameters: bin

528 width $=0.01 \mu \mathrm{m}$, number of timepoints $=6$, jumps to consider $=4, \max$ jump $=1 \mu \mathrm{m}$,

529 gapsAllowed $=0$, and $Z$ correction with $d Z=0.6 \mu \mathrm{m}$. Mean and SD of $F_{\text {bound }}, D_{\text {bound }}$ and $D_{\text {free }}$ were

530 calculated from 3-5 biological replicates.

531 vbSPT (variational Bayesian single-particle tracking) HMM

532 A matlab program running vbSPT HMM ${ }^{63,64}$ (https://gitlab.com/anders.sejr.hansen/anisotropy)

533 was modified to assign each trajectory displacement into two states, 'bound' or 'free'. Then

534 each trajectory was sub-classified as 'bound' if all displacements are classified as bound state;

535 'free' if all displacements are classified as free state; a small fraction of trajectories containing

536 two states were omitted from related analysis. The sub-classified 'bound' and 'free' trajectories

537 from biological replicates of the same conditions were pooled together and used to calculate

538 mean squared displacements (MSD).

539 To calculate the apparent radius of confinement $(\mathrm{Rc})$ for individual trajectories, we fit each MSD

540 curve with the following confined diffusion mode ${ }^{36,104}$,

$$
M S D=R c \times\left(1-e^{\frac{-4 D \Delta t}{R c^{2}}}\right)
$$

\section{Analyzing slow-tracking data}

542 Using the Sojourner R package, the apparent dwell times (temporal length of trajectories) were

543 determined for all "masked" trajectories lasting at least 3 frames.

544 1-CDF curves were generated and fit to a double exponential decay model:

$$
P(t)=f_{s b} e^{-k_{s b} t}+f_{t b} e^{-k_{t b} t}
$$

545 where $\mathrm{k}_{\mathrm{sb}}$ and $\mathrm{k}_{\mathrm{tb}}$ correspond to dissociation rates for stable- and transient-binding events,

546 respectively; $f_{s b}$ and $f_{t b}$ correspond to the fraction of time the molecule spends at stable- and

547 transient-binding sites, respectively, and $f_{s b}+f_{t b}=1$. 
548 The survival curves reflect not only factor dissociation, but also photobleaching, axial and

549 lateral cell or chromatin movements, fluctuating background, etc. To correct for all these

550 factors, assuming that these processes affect Halo-H2B to the same extent as other proteins,

551 and that bulk H2B dissociation is negligible in the experimental time frame of 2-5 min, we

552 measured the apparent unbinding rate for Halo- $\mathrm{H} 2 \mathrm{~B}$ in the same way and used it as a

553 correction factor for other proteins' residence time ${ }^{38}$. The corrected average residence times

554 for stable- $\left(\tau_{\mathrm{sb}}\right)$ and transient-binding $\left(\tau_{\mathrm{tb}}\right)$ were calculated as follows:

$$
\begin{aligned}
\tau_{s b} & =\frac{1}{k_{s b}-k_{s b, H 2 B}} \\
\tau_{t b} & =\frac{1}{k_{t b}-k_{s b, H 2 B}}
\end{aligned}
$$

555 Mean and SD of $k_{s b}, k_{t b}, f_{s b}$ and $f_{t b}$ were calculated from 100 bootstrap samples, then mean and 556 SD of $k_{s b}$ and $k_{t b}$ were used to calculate $\tau_{\mathrm{sb}}$ and $\tau_{\mathrm{tb}}$ with error propagation.

\section{Calculating search kinetics and target occupancy}

558 We integrated approaches from previous studies ${ }^{32,105,106}$ and calculated temporal occupancy as 559 described $^{72}$.

560 First, search time ( $\left.\tau_{\text {search }}\right)$ is the average time it takes from a molecule dissociates off a specific site till it find the next specific site, during which the molecule samples nonspecific sites (each

562 lasts for $\tau_{t b}$ on average) for a number of times $\left(N_{\text {trials }}\right)$ before encountering the next specific

563 target (bound for $\tau_{s b}$ on average). $\tau_{\text {free }}$ is the average free time between two non-specific

564 binding events. Assuming the molecule samples all non-specific and specific sites at random

565 with equal accessibility, i.e., equal probability of binding to all sites, the average search time

566 between two consecutive specific binding events is calculated as:

$$
\tau_{\text {search }}=N_{\text {trials }} \times\left(N_{\text {trials }}+1\right) \times \tau_{\text {free }}
$$

$567 N_{\text {trials }}$ depends on the ratio of number of non-specific $\left(N_{n s}\right)$ to specific sites $\left(N_{s}\right)$, or $r_{s}$ :

$$
N_{\text {trials }}=\frac{N_{s}+N_{n s}}{N_{s}}=1+r_{s}
$$

568 Thus,

$$
\tau_{\text {search }}=\left(1+r_{s}\right) \times \tau_{t b}+\left(2+r_{s}\right) \times \tau_{\text {free }}
$$

569 To determine $r_{s}$, we considered two scenarios underlying detection of binding events during 570 slow tracking ${ }^{72}$. 
To obtain $\tau_{\text {free, }}$ we considered that $F_{\text {bound }}$ is proportional to the fraction of the time a molecule spends bound to chromatin either stably or transiently:

$$
F_{\text {bound }}=\frac{\tau_{s b}+N_{\text {trials }} \times \tau_{t b}}{\tau_{s b}+N_{\text {trials }} \times \tau_{\text {tb }}+\left(N_{\text {trials }}+1\right) \times \tau_{\text {free }}}
$$

583 Thus,

$$
\tau_{\text {free }}=\frac{\frac{\left(1+r_{s}\right) \times \tau_{t b}+\tau_{s b}}{F_{\text {bound }}}-\left(1+r_{s}\right) \times \tau_{t b}-\tau_{s b}}{2+r_{s}}
$$

$584 \tau_{\text {search }}$ was calculated with the values derived for $r_{s}$ and $\tau_{\text {free, }}$ as shown above. We then

585 estimated the sampling interval (SI), the average time between two consecutive binding events 586 at a specific site ${ }^{32}$,

$$
S I=\frac{\left(\tau_{\text {search }}+\tau_{\text {sb }}\right) \times N_{\text {sites }}}{N_{\text {molecules }}}
$$


587 We used $\mathrm{N}_{\text {sites }}$ values presented by GAF and HSF ChIP-seq studies ${ }^{17,48}$. $\mathrm{N}_{\text {molecules }}$ was estimated 588 by flow cytometry (see below). Finally, the average occupancy is the percentage of time a given 589 specific site is occupied by the protein of interest:

$$
\text { Occupancy }=\frac{\tau_{s b}}{S I}
$$

\section{Confocal microscopy}

591 Hemocytes were prepared in the same way described above for live-cell imaging, except for 592 staining with 50 nM JFX554 for 30 min at room temperature or heat shocked on a metal heat 593 block at the indicated temperature (the time for heat shock overlaps with the final stage of 594 staining so that dye labeling and heat shock were completed at the same time). During all 595 incubation steps the sample chambers were covered with aluminum foil to minimize 596 evaporation and block light. Then cells were briefly washed twice with PBS and immediately 597 fixed in freshly made $4 \%$ formaldehyde (diluted from Pierce ${ }^{\mathrm{TM}} 16 \%$ Formaldehyde $(\mathrm{w} / \mathrm{v}$ ), 598 Methanol-free, \#28906) in 1X PBS for $15 \mathrm{~min}$. Fixed samples were washed in PBS for $10 \mathrm{~min}$, 599 then stained with DAPI for $10 \mathrm{~min}$ and washed in PBS for $5 \mathrm{~min}$. Sample chambers were filled 600 with PBS and covered by a coverglass on top. Salivary glands were dissected, then stained and 601 heat-shocked similarly as hemocytes except that JF554 staining was performed after fixation. 602 Salivary gland samples were mounted on glass slides in VECTASHIELD ${ }^{\circledR}$ Antifade Mounting 603 Medium (Vector Labs H-1000-10). We imaged these samples on a LSM800 Airyscan confocal 604 microscope (Zeiss, Germany) with a 63X objective. Z-stacks were taken with $0.2 \mu \mathrm{m}$ step size for 605 hemocyte nuclei and $0.5 \mu \mathrm{m}$ step size for salivary gland polytene nuclei. The same laser and 606 scanning settings were used between samples in the same experiment.

\section{Estimation of cellular abundances for Halo-tagged proteins by flow cytometry}

608 Cellular abundances of Halo-GAF and HSF-Halo were estimated by flow cytometry using a 609 calibrated C32 CTCF-Halo U2OS human cell line ${ }^{81,107}$. U2OS cell samples were prepared as 610 described $^{107}$ with minor modifications. Briefly, we labeled WT and CTCF-Halo U2OS cells with 50 $611 \mathrm{nM} \mathrm{JF552}$ for $30 \mathrm{~min}$ at $37^{\circ} \mathrm{C} / 5 \% \mathrm{CO}_{2}$ in a tissue-culture incubator, washed out the dye

612 (removed medium; rinsed with PBS, and incubated with fresh media for $5 \mathrm{~min}$ in the incubator)

613 and then immediately prepared cells for flow cytometry. Resuspended cells were filtered

614 through a $40 \mu \mathrm{m}$ filter and placed on ice until their fluorescence was read out by the flow 615 cytometer. To prepare fly hemocytes for flow cytometry, 20 30 3rd instar larvae were 616 thoroughly washed and dissected on ice in a $1.5 \mathrm{~mL}$ eppendorf tube lid containing Schneider's 617 Drosophila medium (Gibco ${ }^{\text {TM }}$ 21720024), EDTA-free protease inhibitor cocktail (Roche 618 4693159001) and 10\% FBS (HyClone FBS SH30910.03, Cytiva, MA). Hemocytes were collected 619 and stored on ice. After dissection was done for all fly strains, hemocytes were stained with $62050 \mathrm{nM}$ JF552 in $1 \mathrm{~mL}$ medium at room temperature for $30 \mathrm{~min}$. All tubes were pre-rinsed with 621 FBS to minimize cell stickiness to the tubes. After staining, hemocytes were centrifuged at $200 \mathrm{x}$ $622 \mathrm{~g}$ for $5 \mathrm{~min}$, resuspended in $1 \mathrm{~mL}$ fresh medium and washed at room temperature for $15 \mathrm{~min}$ 
623 (longer wash time than for U2OS cells to remove non-specific cytoplasmic signals). The

624 hemocytes were centrifuged again, resuspended in $500 \mu \mathrm{L}$ medium and placed on ice until their

625 fluorescence was read out by the flow cytometer.

626 We used a SH800 (Sony, Japan) cell sorter in analyze mode to measure fluorescence intensity in 627 the U2OS cell lines and hemocytes with the same settings. Single live cells were gated using

628 forward and side scattering. JF552 fluorescence was excited using a $561 \mathrm{~nm}$ laser and emission

629 read out using a $617 / 30$ band pass filter. The absolute abundance of protein of interest $N_{\text {molecuels }}$

630 (mean number of molecules per cell) was obtained according to:

$$
N_{\text {molecules }}=\frac{I-I_{\text {Background }}}{I_{C T C F}-I_{U 2 \text { OSBackground }}} \times N_{C T C F}
$$

631 Where $I$ is the average measured fluorescence intensity of the cells expressing the protein of

632 interest, $I_{\text {Background }}$ is the average measured fluorescence intensity of hemocytes from a fly strain 633 not expressing HaloTag (w1118), $I_{\text {CTCF }}$ is the average measured fluorescence intensity of the 634 CTCF-Halo standard U2OS cell line, IU2OSBackground is the average measured fluorescence intensity 635 of WT U2OS cells not expressing HaloTag, and $N_{\text {CTCF }}$ is the absolute abundance of CTCF-Halo 636 ( 109,800 proteins per cell). For each experiment, hemocytes and U2OS cells were stained with 637 the same aliquot of JF552 stock solution and measured during the same flow cytometry session. 638 We performed three to five biological replicates to get mean and standard deviation values.

639 Characterization of cell cycle stage for larval hemocytes using the Fly-Fucci system

640 A fly strain with the Fly-Fucci markers under UAS control (UAS-CFP.E2f1.1-230 and UAS641 Venus.NLS.CycB.1-266, BDSC:55122) was crossed to a hemocyte-specific driver Cg-Gal4 642 (BDSC:7011). $3^{\text {rd }}$ instar larvae were washed then dissected to release hemocytes in a drop of 643 PBS on a slide, then the fluorescence of hemocytes were imaged immediately on a Zeiss 644 Axioplan 2 compound microscope. 


\section{ACKNOWLEDGEMENTS}

646 We thank Anders Hansen and Maxime Woringer for discussion on Spot-On analyses, Anders

647 Hansen, Claudia Cattoglio, Xavier Darzacq and Robert Tjian for the CTCF-Halo U2OS cell line and

648 discussions on measuring factor abundance, Tim Lionnet, James Zhe Liu, Peng Dong, and Brian

649 Mehl for advice on SPT, Yick Hin Ling and Sun Jay Yoo for assistance with data analysis, Pascal

650 Vallotton for customization of DiaTrack software, Paul Badenhorst for advice on larval

651 hemocyte culture, Steve Deluca for advice on bioinformatics, Weina Dai for imaging assistance,

652 Erin Pryce and the Integrated Imaging Center for LSM 800 training, Yundong Liu for assembly

653 and maintenance of a high performance computational platform, John Lis, Mike Levine, and

654 Gordon Hager for discussion, and Wu Lab members for comments on the manuscript. This

655 study was supported by HHMI funding to the TIC (C.W., Q.Z., and L.L.), Johns Hopkins

656 Bloomberg Distinguished Professorship funds (C.W.), and National Institutes of Health grants

657 GM132290-01 (C.W.) and DK127432 (C.W.).

658

659

\section{AUTHOR CONTRIBUTIONS}

660 X.T. performed all genetic and imaging experiments with support from T.L., J.W., and Y.R. and

661 all data analysis using R functions created by S.L. and X.T.. L.D.L. and Q.Z. synthesized

662 JF552/JFX554 and JF700. X.T. and C.W. designed the study and wrote the paper with input from

663 all authors.

664

665

\section{DECLARATION OF INTERESTS}

666 L.D.L. and Q.Z. are listed as inventors on patents and patent applications whose values might be 667 affected by publication.

668

669

670

\section{REFERENCES}

671 1. Biggin, M. D. \& Tjian, R. Transcription factors that activate the Ultrabithorax promoter in

672 developmentally staged extracts. Cell 53, 699-711 (1988).

673 2. Gilmour, D. S., Thomas, G. H. \& Elgin, S. C. Drosophila nuclear proteins bind to regions of

674 alternating $C$ and T residues in gene promoters. Science 245, 1487-1490 (1989).

675 3. Farkas, G. et al. The Trithorax-like gene encodes the Drosophila GAGA factor. Nature 371, 676 806-808 (1994).

677 4. Soeller, W. C., Oh, C. E. \& Kornberg, T. B. Isolation of cDNAs encoding the Drosophila GAGA 678 transcription factor. Mol. Cell. Biol. 13, 7961-7970 (1993). 
5. Chopra, V. S. et al. Transcriptional activation by GAGA factor is through its direct interaction with dmTAF3. Dev. Biol. 317, 660-670 (2008).

6. Lomaev, D. et al. The GAGA factor regulatory network: Identification of GAGA factor associated proteins. PLoS One 12, e0173602 (2017).

7. Giot, L. et al. A protein interaction map of Drosophila melanogaster. Science $302,1727-$ 1736 (2003).

8. Li, J. et al. Kinetic competition between elongation rate and binding of NELF controls promoter-proximal pausing. Mol. Cell 50, 711-722 (2013).

9. Croston, G. E., Kerrigan, L. A., Lira, L. M., Marshak, D. R. \& Kadonaga, J. T. Sequencespecific antirepression of histone H1-mediated inhibition of basal RNA polymerase II transcription. Science 251, 643-649 (1991).

10. Cirillo, L. A. \& Zaret, K. S. An early developmental transcription factor complex that is more stable on nucleosome core particles than on free DNA. Mol. Cell 4, 961-969 (1999).

11. Soufi, A. et al. Pioneer transcription factors target partial DNA motifs on nucleosomes to initiate reprogramming. Cell 161, 555-568 (2015).

12. Tsukiyama, T., Becker, P. B. \& Wu, C. ATP-dependent nucleosome disruption at a heatshock promoter mediated by binding of GAGA transcription factor. Nature 367, 525-532 (1994).

13. Okada, M. \& Hirose, S. Chromatin remodeling mediated by Drosophila GAGA factor and ISWI activates fushi tarazu gene transcription in vitro. Mol. Cell. Biol. 18, 2455-2461 (1998).

14. Nakayama, T., Shimojima, T. \& Hirose, S. The PBAP remodeling complex is required for histone H3.3 replacement at chromatin boundaries and for boundary functions. Development 139, 4582-4590 (2012).

15. Xiao, H. et al. Dual functions of largest NURF subunit NURF301 in nucleosome sliding and transcription factor interactions. Mol. Cell 8, 531-543 (2001).

16. Sherwood, R. I. et al. Discovery of directional and nondirectional pioneer transcription factors by modeling DNase profile magnitude and shape. Nat. Biotechnol. 32, 171-178 (2014).

17. Fuda, N. J. et al. GAGA factor maintains nucleosome-free regions and has a role in RNA polymerase II recruitment to promoters. PLoS Genet. 11, e1005108 (2015).

18. Duarte, F. M. et al. Transcription factors GAF and HSF act at distinct regulatory steps to modulate stress-induced gene activation. Genes Dev. 30, 1731-1746 (2016).

19. Badenhorst, P., Voas, M., Rebay, I. \& Wu, C. Biological functions of the ISWI chromatin remodeling complex NURF. Genes Dev. 16, 3186-3198 (2002).

20. Boija, A. et al. CBP Regulates Recruitment and Release of Promoter-Proximal RNA Polymerase II. Mol. Cell 68, 491-503.e5 (2017). 
21. Kuroda, M. I., Kang, H., De, S. \& Kassis, J. A. Dynamic Competition of Polycomb and Trithorax in Transcriptional Programming. Annu. Rev. Biochem. 89, 235-253 (2020).

22. Wolle, D. et al. Functional Requirements for Fab-7 Boundary Activity in the Bithorax Complex. Mol. Cell. Biol. 35, 3739-3752 (2015).

23. van Steensel, B., Delrow, J. \& Bussemaker, H. J. Genomewide analysis of Drosophila GAGA factor target genes reveals context-dependent DNA binding. Proc. Natl. Acad. Sci. U. S. A. 100, 2580-2585 (2003).

24. Judd, J., Duarte, F. M. \& Lis, J. T. Pioneer-like factor GAF cooperates with PBAP (SWI/SNF) and NURF (ISWI) to regulate transcription. Genes Dev. 35, 147-156 (2021).

25. Gaskill, M. M., Gibson, T. J., Larson, E. D. \& Harrison, M. M. GAF is essential for zygotic genome activation and chromatin accessibility in the early embryo. Elife 10, (2021).

26. Benyajati, C. et al. Multiple isoforms of GAGA factor, a critical component of chromatin structure. Nucleic Acids Res. 25, 3345-3353 (1997).

27. Greenberg, A. J. \& Schedl, P. GAGA factor isoforms have distinct but overlapping functions in vivo. Mol. Cell. Biol. 21, 8565-8574 (2001).

28. Tepass, U., Fessler, L. I., Aziz, A. \& Hartenstein, V. Embryonic origin of hemocytes and their relationship to cell death in Drosophila. Development 120, 1829-1837 (1994).

29. Read, D., Butte, M. J., Dernburg, A. F., Frasch, M. \& Kornberg, T. B. Functional studies of the BTB domain in the Drosophila GAGA and Mod(mdg4) proteins. Nucleic Acids Res. 28, 3864-3870 (2000).

30. Rust, M. J., Bates, M. \& Zhuang, X. Sub-diffraction-limit imaging by stochastic optical reconstruction microscopy (STORM). Nat. Methods 3, 793-795 (2006).

31. Heilemann, M. et al. Subdiffraction-resolution fluorescence imaging with conventional fluorescent probes. Angew. Chem. Int. Ed Engl. 47, 6172-6176 (2008).

32. Chen, J. et al. Single-molecule dynamics of enhanceosome assembly in embryonic stem cells. Cell 156, 1274-1285 (2014).

33. Hansen, A. S. et al. Robust model-based analysis of single-particle tracking experiments with Spot-On. Elife 7, (2018).

34. Chong, S. et al. Imaging dynamic and selective low-complexity domain interactions that control gene transcription. Science 361, (2018).

35. Swinstead, E. E. et al. Steroid Receptors Reprogram FoxA1 Occupancy through Dynamic Chromatin Transitions. Cell 165, 593-605 (2016).

36. Lerner, J. et al. Two-Parameter Mobility Assessments Discriminate Diverse Regulatory Factor Behaviors in Chromatin. Mol. Cell 79, 677-688.e6 (2020).

37. Liu, H. et al. Visualizing long-term single-molecule dynamics in vivo by stochastic protein labeling. Proc. Natl. Acad. Sci. U. S. A. 115, 343-348 (2018).

38. Hansen, A. S., Pustova, I., Cattoglio, C., Tjian, R. \& Darzacq, X. CTCF and cohesin regulate 
chromatin loop stability with distinct dynamics. Elife 6, (2017).

39. Espinás, M. L. et al. The N-terminal POZ domain of GAGA mediates the formation of oligomers that bind DNA with high affinity and specificity. J. Biol. Chem. 274, 16461-16469 (1999).

40. Katsani, K. R., Hajibagheri, M. A. \& Verrijzer, C. P. Co-operative DNA binding by GAGA transcription factor requires the conserved $\mathrm{BTB} / \mathrm{POZ}$ domain and reorganizes promoter topology. EMBO J. 18, 698-708 (1999).

41. Wilkins, R. C. \& Lis, J. T. DNA distortion and multimerization: novel functions of the glutamine-rich domain of GAGA factor. J. Mol. Biol. 285, 515-525 (1999).

42. Tsukiyama, T. \& Wu, C. Purification and properties of an ATP-dependent nucleosome remodeling factor. Cell 83, 1011-1020 (1995).

43. Badenhorst, P. et al. The Drosophila nucleosome remodeling factor NURF is required for Ecdysteroid signaling and metamorphosis. Genes Dev. 19, 2540-2545 (2005).

44. Chalkley, G. E. et al. The transcriptional coactivator SAYP is a trithorax group signature subunit of the PBAP chromatin remodeling complex. Mol. Cell. Biol. 28, 2920-2929 (2008).

45. Yen, K., Vinayachandran, V., Batta, K., Koerber, R. T. \& Pugh, B. F. Genome-wide nucleosome specificity and directionality of chromatin remodelers. Cell 149, 1461-1473 (2012).

46. Shopland, L. S., Hirayoshi, K., Fernandes, M. \& Lis, J. T. HSF access to heat shock elements in vivo depends critically on promoter architecture defined by GAGA factor, TFIID, and RNA polymerase II binding sites. Genes Dev. 9, 2756-2769 (1995).

47. Lis, J. T., Mason, P., Peng, J., Price, D. H. \& Werner, J. P-TEFb kinase recruitment and function at heat shock loci. Genes Dev. 14, 792-803 (2000).

48. Guertin, M. J. \& Lis, J. T. Chromatin landscape dictates HSF binding to target DNA elements. PLoS Genet. 6, e1001114 (2010).

49. Lis, J. \& Wu, C. Protein traffic on the heat shock promoter: parking, stalling, and trucking along. Cell 74, 1-4 (1993).

50. Vuister, G. W. et al. Solution structure of the DNA-binding domain of Drosophila heat shock transcription factor. Nat. Struct. Biol. 1, 605-614 (1994).

51. Kim, S. J., Tsukiyama, T., Lewis, M. S. \& Wu, C. Interaction of the DNA-binding domain of Drosophila heat shock factor with its cognate DNA site: a thermodynamic analysis using analytical ultracentrifugation. Protein Sci. 3, 1040-1051 (1994).

52. Westwood, J. T., Clos, J. \& Wu, C. Stress-induced oligomerization and chromosomal relocalization of heat-shock factor. Nature 353, 822-827 (1991).

53. Baler, R., Dahl, G. \& Voellmy, R. Activation of human heat shock genes is accompanied by oligomerization, modification, and rapid translocation of heat shock transcription factor HSF1. Mol. Cell. Biol. 13, 2486-2496 (1993). 
54. Sarge, K. D., Murphy, S. P. \& Morimoto, R. I. Activation of heat shock gene transcription by heat shock factor 1 involves oligomerization, acquisition of DNA-binding activity, and nuclear localization and can occur in the absence of stress. Mol. Cell. Biol. 13, 1392-1407 (1993).

55. Perisic, O., Xiao, H. \& Lis, J. T. Stable binding of Drosophila heat shock factor to head-tohead and tail-to-tail repeats of a conserved 5 bp recognition unit. Cell 59, 797-806 (1989).

56. Neudegger, T., Verghese, J., Hayer-Hartl, M., Hartl, F. U. \& Bracher, A. Structure of human heat-shock transcription factor 1 in complex with DNA. Nat. Struct. Mol. Biol. 23, 140-146 (2016).

57. Westwood, J. T. \& Wu, C. Activation of Drosophila heat shock factor: conformational change associated with a monomer-to-trimer transition. Mol. Cell. Biol. 13, 3481-3486 (1993).

58. Spradling, A. C. et al. The Berkeley Drosophila Genome Project gene disruption project: Single P-element insertions mutating $25 \%$ of vital Drosophila genes. Genetics 153, 135-177 (1999).

59. Jedlicka, P., Mortin, M. A. \& Wu, C. Multiple functions of Drosophila heat shock transcription factor in vivo. EMBO J. 16, 2452-2462 (1997).

60. Yao, J., Munson, K. M., Webb, W. W. \& Lis, J. T. Dynamics of heat shock factor association with native gene loci in living cells. Nature 442, 1050-1053 (2006).

61. Chowdhary, S., Kainth, A. S., Pincus, D. \& Gross, D. S. Heat Shock Factor 1 Drives Intergenic Association of Its Target Gene Loci upon Heat Shock. Cell Rep. 26, 18-28.e5 (2019).

62. Gaglia, G. et al. HSF1 phase transition mediates stress adaptation and cell fate decisions. Nat. Cell Biol. 22, 151-158 (2020).

63. Persson, F., Lindén, M., Unoson, C. \& Elf, J. Extracting intracellular diffusive states and transition rates from single-molecule tracking data. Nat. Methods 10, 265-269 (2013).

64. Hansen, A. S., Amitai, A., Cattoglio, C., Tjian, R. \& Darzacq, X. Guided nuclear exploration increases CTCF target search efficiency. Nat. Chem. Biol. 16, 257-266 (2020).

65. Zentner, G. E. \& Henikoff, S. High-resolution digital profiling of the epigenome. Nat. Rev. Genet. 15, 814-827 (2014).

66. Kasinathan, S., Orsi, G. A., Zentner, G. E., Ahmad, K. \& Henikoff, S. High-resolution mapping of transcription factor binding sites on native chromatin. Nat. Methods 11, 203-209 (2014).

67. Tilly, B. C. et al. In vivo analysis reveals that ATP-hydrolysis couples remodeling to SWI/SNF release from chromatin. Elife 10, (2021).

68. Iurlaro, M. et al. Mammalian SWI/SNF continuously restores local accessibility to chromatin. Nat. Genet. 53, 279-287 (2021).

69. Schick, S. et al. Acute BAF perturbation causes immediate changes in chromatin accessibility. Nat. Genet. 53, 269-278 (2021). 
828

829

830

831

832

833

834

835

836

837

838

839

840

841

842

843

844

845

846

847

848

849

850

851

852

853

854

855

856

857

858

859

860

861

862

863

864

70. Kim, J. M. et al. Single-molecule imaging of chromatin remodelers reveals role of ATPase in promoting fast kinetics of target search and dissociation from chromatin. Elife 10, (2021).

71. Jeronimo, C. et al. FACT is recruited to the +1 nucleosome of transcribed genes and spreads in a Chd1-dependent manner. Mol. Cell (2021) doi:10.1016/j.molcel.2021.07.010.

72. Nguyen, V. Q. et al. Spatiotemporal coordination of transcription preinitiation complex assembly in live cells. Mol. Cell (2021) doi:10.1016/j.molcel.2021.07.022.

73. Kwon, S. Y., Grisan, V., Jang, B., Herbert, J. \& Badenhorst, P. Genome-Wide Mapping Targets of the Metazoan Chromatin Remodeling Factor NURF Reveals Nucleosome Remodeling at Enhancers, Core Promoters and Gene Insulators. PLoS Genet. 12, e1005969 (2016).

74. Liu, Z. \& Tjian, R. Visualizing transcription factor dynamics in living cells. J. Cell Biol. 217, 1181-1191 (2018).

75. Wall, G., Varga-Weisz, P. D., Sandaltzopoulos, R. \& Becker, P. B. Chromatin remodeling by GAGA factor and heat shock factor at the hypersensitive Drosophila hsp26 promoter in vitro. EMBO J. 14, 1727-1736 (1995).

76. Chetverina, D., Erokhin, M. \& Schedl, P. GAGA factor: a multifunctional pioneering chromatin protein. Cell. Mol. Life Sci. 78, 4125-4141 (2021).

77. von Hippel, P. H. \& Berg, O. G. Facilitated target location in biological systems. J. Biol. Chem. 264, 675-678 (1989).

78. Schneider, I. Cell lines derived from late embryonic stages of Drosophila melanogaster. J. Embryol. Exp. Morphol. 27, 353-365 (1972).

79. Cherbas, L. et al. The transcriptional diversity of 25 Drosophila cell lines. Genome Res. 21, 301-314 (2011).

80. Tsai, S.-Y., Chang, Y.-L., Swamy, K. B. S., Chiang, R.-L. \& Huang, D.-H. GAGA factor, a positive regulator of global gene expression, modulates transcriptional pausing and organization of upstream nucleosomes. Epigenetics Chromatin 9, 32 (2016).

81. Cattoglio, C. et al. Determining cellular CTCF and cohesin abundances to constrain 3D genome models. elife vol. 8 (2019).

82. Schwendemann, A. \& Lehmann, M. Pipsqueak and GAGA factor act in concert as partners at homeotic and many other loci. Proc. Natl. Acad. Sci. U. S. A. 99, 12883-12888 (2002).

83. Gutierrez-Perez, I. et al. Ecdysone-Induced 3D Chromatin Reorganization Involves Active Enhancers Bound by Pipsqueak and Polycomb. Cell Rep. 28, 2715-2727.e5 (2019).

84. Duan, J. et al. CLAMP and Zelda function together to promote zygotic genome activation. Elife 10, (2021).

85. Kaye, E. G. et al. Differential Occupancy of Two GA-Binding Proteins Promotes Targeting of the Drosophila Dosage Compensation Complex to the Male X Chromosome. Cell Rep. 22, 3227-3239 (2018). 
86. Sekiya, T., Muthurajan, U. M., Luger, K., Tulin, A. V. \& Zaret, K. S. Nucleosome-binding affinity as a primary determinant of the nuclear mobility of the pioneer transcription factor FoxA. Genes Dev. 23, 804-809 (2009).

87. Brodsky, S. et al. Intrinsically Disordered Regions Direct Transcription Factor In Vivo Binding Specificity. Mol. Cell 79, 459-471.e4 (2020).

88. Chaharbakhshi, E. \& Jemc, J. C. Broad-complex, tramtrack, and bric-à-brac (BTB) proteins: Critical regulators of development. Genesis 54, 505-518 (2016).

89. Mahmoudi, T., Katsani, K. R. \& Verrijzer, C. P. GAGA can mediate enhancer function in trans by linking two separate DNA molecules. EMBO J. 21, 1775-1781 (2002).

90. Paakinaho, V., Johnson, T. A., Presman, D. M. \& Hager, G. L. Glucocorticoid receptor quaternary structure drives chromatin occupancy and transcriptional outcome. Genome Res. 29, 1223-1234 (2019).

91. Biggin, M. D. Animal transcription networks as highly connected, quantitative continua. Dev. Cell 21, 611-626 (2011).

92. Fisher, W. W. et al. DNA regions bound at low occupancy by transcription factors do not drive patterned reporter gene expression in Drosophila. Proc. Natl. Acad. Sci. U. S. A. 109, 21330-21335 (2012).

93. Zaret, K. S. Pioneer Transcription Factors Initiating Gene Network Changes. Annu. Rev. Genet. 54, 367-385 (2020).

94. Hansen, J. L. \& Cohen, B. A. A Test of the Pioneer Factor Hypothesis. bioRxiv 2021.08.17.456650 (2021) doi:10.1101/2021.08.17.456650.

95. Johnson, T. A. et al. Conventional and pioneer modes of glucocorticoid receptor interaction with enhancer chromatin in vivo. Nucleic Acids Res. 46, 203-214 (2018).

96. Heinz, S. et al. Simple combinations of lineage-determining transcription factors prime cisregulatory elements required for macrophage and B cell identities. Mol. Cell 38, 576-589 (2010).

97. Bier, E., Harrison, M. M., O’Connor-Giles, K. M. \& Wildonger, J. Advances in Engineering the Fly Genome with the CRISPR-Cas System. Genetics 208, 1-18 (2018).

98. Sharan, S. K., Thomason, L. C., Kuznetsov, S. G. \& Court, D. L. Recombineering: a homologous recombination-based method of genetic engineering. Nat. Protoc. 4, 206-223 (2009).

99. Zhang, Y., Schreiner, W. \& Rong, Y. S. Genome manipulations with bacterial recombineering and site-specific integration in Drosophila. Methods Mol. Biol. 1114, 11-24 (2014).

100. Groth, A. C., Fish, M., Nusse, R. \& Calos, M. P. Construction of transgenic Drosophila by using the site-specific integrase from phage phiC31. Genetics 166, 1775-1782 (2004).

101. Ranjan, A. et al. Live-cell single particle imaging reveals the role of RNA polymerase II in histone H2A.Z eviction. Elife 9, (2020). 
903

904

905

906

907

908

909

910

911

912

913

914

915

916

917

918

919

920

921

922

923

924

925

102. Schindelin, J. et al. Fiji: an open-source platform for biological-image analysis. Nat. Methods 9, 676-682 (2012).

103. Vallotton, P. \& Olivier, S. Tri-track: free software for large-scale particle tracking. Microsc. Microanal. 19, 451-460 (2013).

104. Wieser, S. \& Schütz, G. J. Tracking single molecules in the live cell plasma membrane-Do's and Don't's. Methods 46, 131-140 (2008).

105. Loffreda, A. et al. Live-cell p53 single-molecule binding is modulated by C-terminal acetylation and correlates with transcriptional activity. Nat. Commun. 8, 313 (2017).

106. Tatavosian, R. et al. Live-cell single-molecule dynamics of PcG proteins imposed by the DIPG H3.3K27M mutation. Nat. Commun. 9, 2080 (2018).

107. Cattoglio, C., Darzacq, X., Tjian, R. \& Hansen, A. S. Estimating Cellular Abundances of Halotagged Proteins in Live Mammalian Cells by Flow Cytometry. Bio Protoc 10, e3527 (2020).

108. Omichinski, J. G., Pedone, P. V., Felsenfeld, G., Gronenborn, A. M. \& Clore, G. M. The solution structure of a specific GAGA factor-DNA complex reveals a modular binding mode. Nat. Struct. Biol. 4, 122-132 (1997).

109. Ramírez, F. et al. deepTools2: a next generation web server for deep-sequencing data analysis. Nucleic Acids Res. 44, W160-5 (2016).

110. Wilkins, R. C. \& Lis, J. T. GAGA factor binding to DNA via a single trinucleotide sequence element. Nucleic Acids Res. 26, 2672-2678 (1998).

111. Zielke, N. et al. Fly-FUCCl: A versatile tool for studying cell proliferation in complex tissues. Cell Rep. 7, 588-598 (2014).

112. Asha, H. et al. Analysis of Ras-induced overproliferation in Drosophila hemocytes. Genetics 163, 203-215 (2003). 


\section{Figure 1}
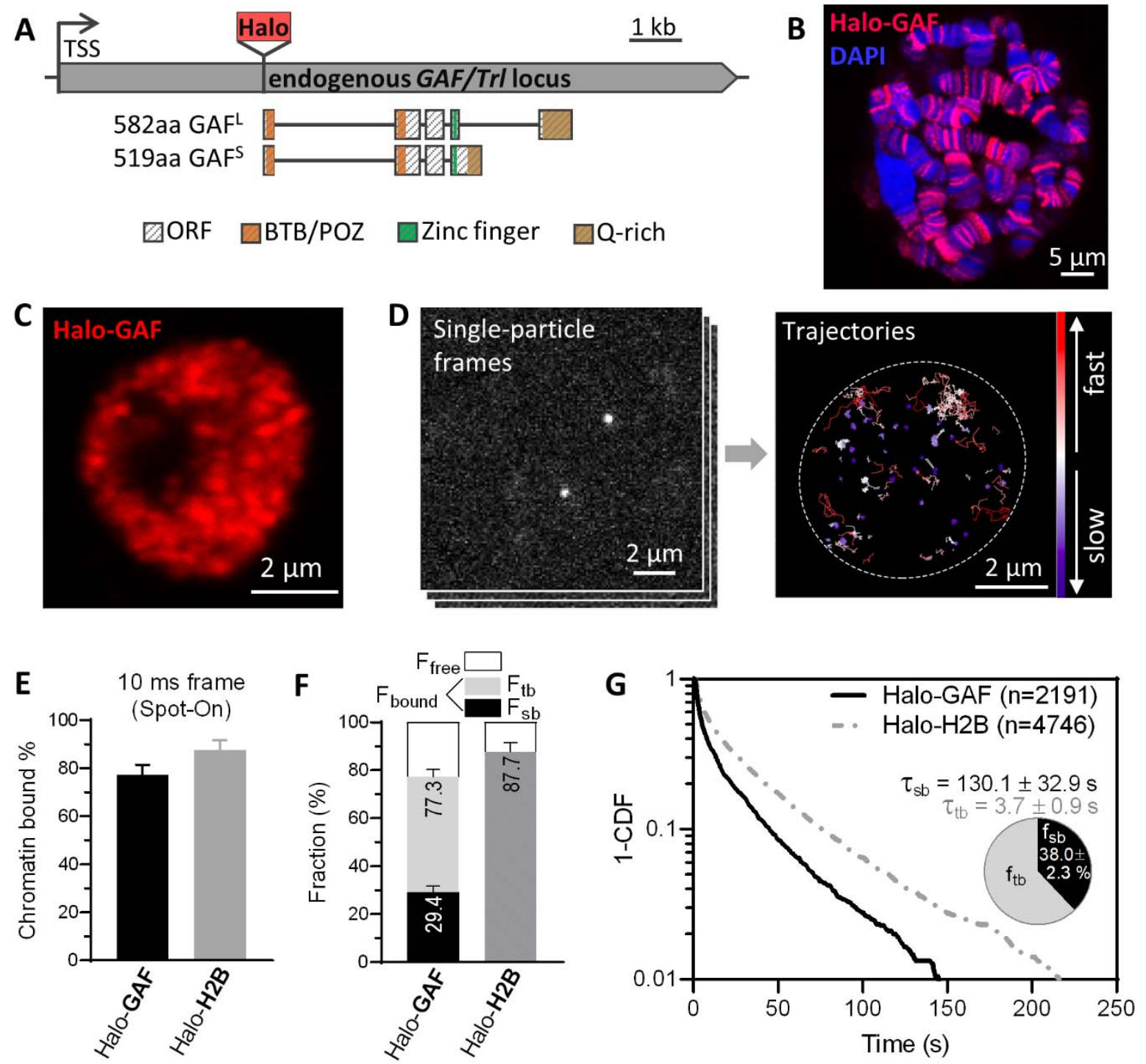

927 Figure 1. Chromatin-binding dynamics of GAF shown by single-particle tracking (SPT) in live 928 Drosophila hemocytes.

(A) Diagram of HaloTag (Halo) knock-in at the N-terminus of endogenous GAF/Trl locus. Protein domains in color. TSS, transcription start site. C-terminal GAF-HaloTag fusions for two splicing isoforms $\mathrm{GAF}^{\mathrm{S}}$ (short) and $\mathrm{GAF}^{\mathrm{L}}$ (long) are shown in Fig S1.

(B) Halo-GAF binds to specific polytene chromosome loci in fixed $3^{\text {rd }}$ instar larval salivary gland nuclei, revealed by JF552 fluorescence (red); DNA counterstained with DAPI (blue).

(C) Confocal distribution of Halo-GAF (JF552) foci in fixed 3rd instar larval hemocyte nuclei (predominantly diploid plasmatocytes in G2 phase of the cell cycle). 
(D) SMT frames and superimposed trajectories of Halo-GAF in live hemocytes, color-coded according to diffusion coefficients. Dashed oval marks the nucleus.

(E) Spot-On kinetic modeling of fast-tracking data shows chromatin-bound and free fractions for Halo-GAF and Halo-H2B. Results are mean \pm SD from three biological replicates.

941

(F) Chromatin-free fraction $\left(F_{\text {free }}\right)$, global stable- and transient-binding fractions $\left(F_{s b}\right.$ and $\left.F_{t b}\right)$ of Halo-GAF extracted from fast- and slow-tracking data in (E) and (G), with error propagation.

(G) Survival probability curves (1-CDF) plotted from apparent dwell times of thousands ( $\mathrm{n}$ ) of single-particle chromatin-binding events for Halo-GAF. Average residence times for stable- $\left(\tau_{s b}\right)$ and transient- $\left(\tau_{t b}\right)$ binding by Halo-GAF are corrected by using Halo-H2B as a 'non-dissociating' standard. Pie charts show stable- $\left(f_{s b}\right)$ and transient-binding $\left(f_{t b}\right)$ fractions. Mean and SD provided. Errors represent bootstrapped SD. 


\section{Figure 2}

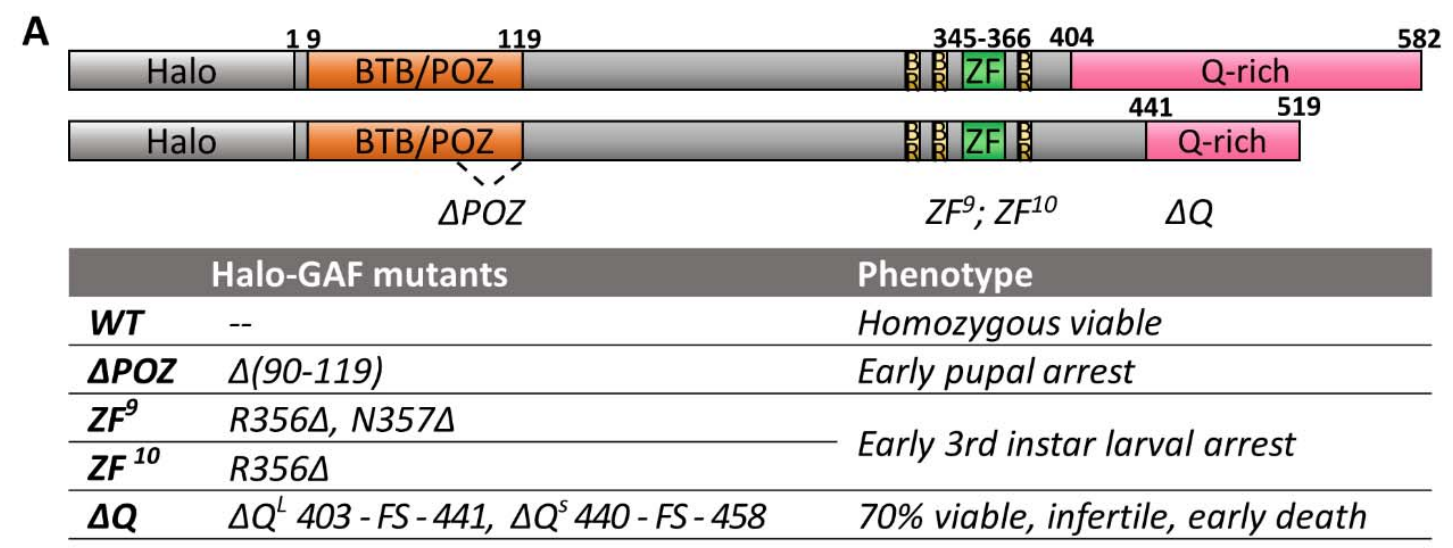
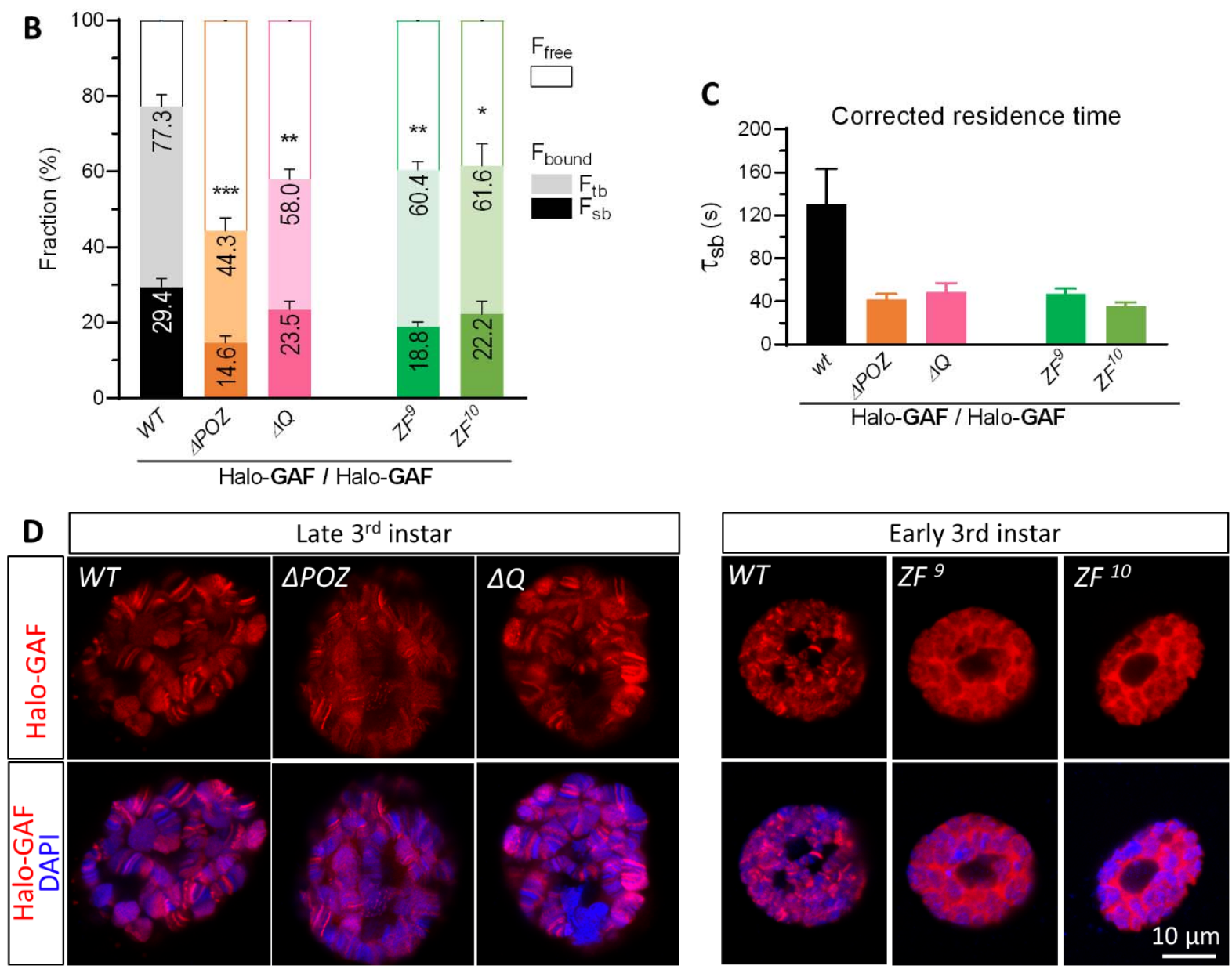
(A) Schematics of Halo-GAF (long) and Halo-GAF ${ }^{\mathrm{S}}$ (short) isoforms with functional domains to scale. BTB/POZ: Broad-complex, Tramtrack and Bric-à-brac/Poxvirus and Zinc finger, BR: basic region, ZF: zinc finger, Q-rich: glutamine-rich. Halo-GAF deletions were generated by CRISPR-Cas9. $\triangle P O Z$ contains a $90 \mathrm{bp}$ deletion in the second exon, generating a 30-AA deletion ( $\triangle 90-119)$ of the POZ domain, which includes G93 and L112 that are essential for transcription activation ${ }^{29}$. The deleted Arg and Asn amino acids in the zinc finger $\left(Z F^{9}, Z F^{10}\right)$ make contact with 'GAG' of the consensus GAF binding site(Omichinski et al. 1997). $\Delta Q$ contains small deletions at the beginning of Q-rich domains of both long and short isoforms, resulting in frame shift and truncation of $Q$ rich domains. Table reports mutant phenotypes. FS: frameshift.

(B) Global chromatin-bound and free fractions (\%) for wild type (WT) and mutant Halo-GAF. Fast and slow-tracking results with propagated errors. ${ }^{*}, p<0.05 ; * *, p<0.01 ; * * *, p<$ 0.001 , unpaired $t$-test for fast-tracking $(n=3-4)$.

(C) Average residence times for WT and mutant Halo-GAF, corrected as in Fig. 1. Error bars represent bootstrapped SD.

(D) Halo-GAF distribution on fixed salivary gland (SG) polytene nuclei for $W T$ and GAF mutants. $\triangle P O Z$ and $\triangle Q$ nuclei are from late 3rd instar larvae with $W T$ nuclei from the same stage as control. $Z F^{9}$ and $Z F^{10}$ polytene nuclei are from early instar larvae with $W T$ nuclei from the same stage as control. Red: Halo-GAF; blue: DAPI. One representative confocal z-section is shown. 


\section{Figure 3}
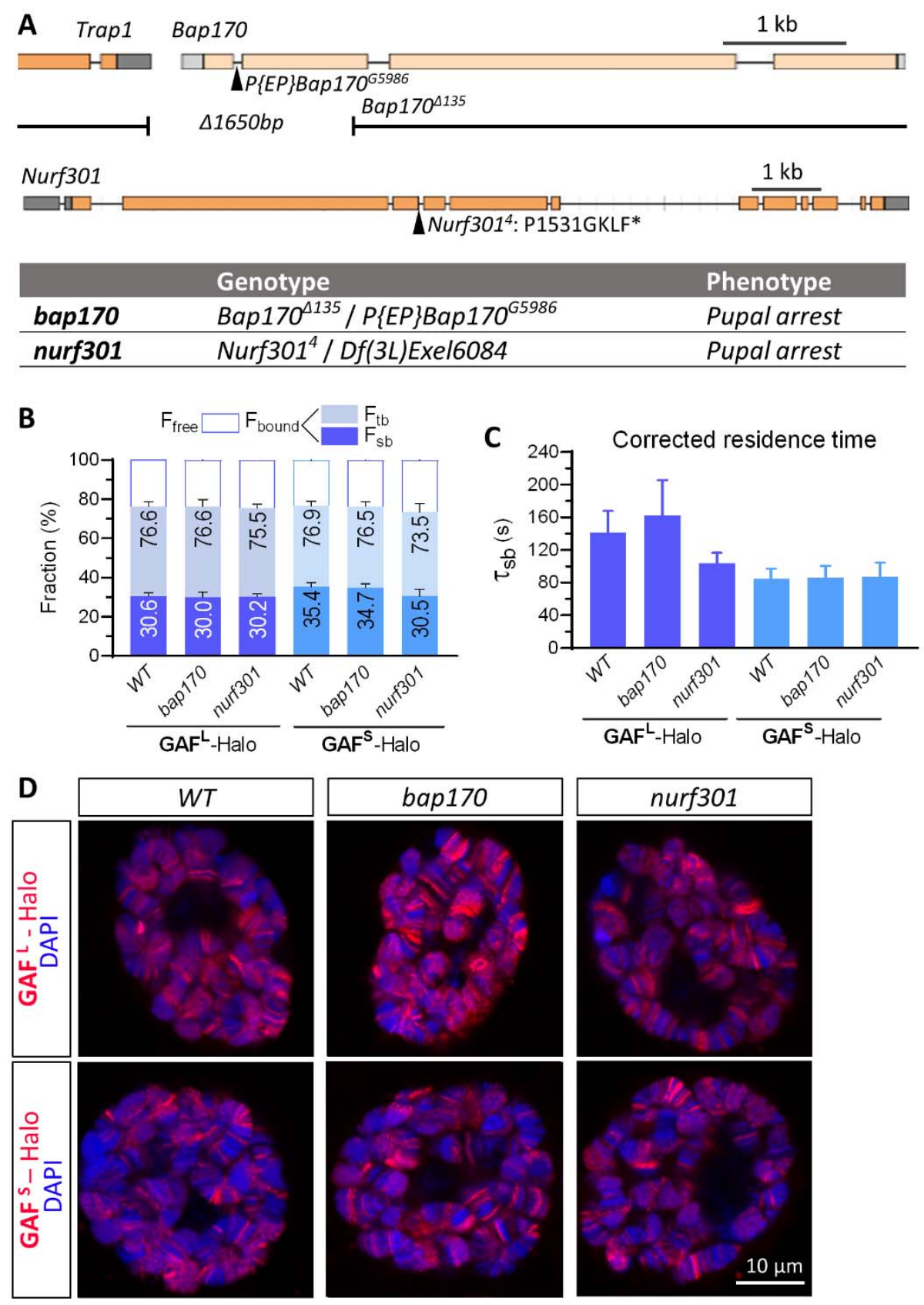

973 Figure 3. Chromatin binding by GAF is largely independent of remodelers PBAP and NURF.

(A) Bap170 and Nurf301/E(bx) mutants and phenotypes. Bap170 135 harbors a $1650 \mathrm{bp}$ deletion spanning the promoter, 5'UTR and first two exons of Bap170, P\{EP\}Bap170 $0^{G 5986}$ has a $P\{E P\}$ element insertion at 447 bp downstream from the transcription start site in the first intron of the gene. Bap170 135 abolishes Bap170 expression and strongly reduces polybromo protein level at larval stage, causing pupal lethality when 
homozygous(Carrera et al. 2008), while flies homozygous for the $P\{E P\} B a p 170^{G 5986}$ die primarily as pharate adults, with only $3 \%$ male homozygous adults(Chalkley et al. 2008). Transheterozygous Bap170 1135 / P\{EP\}Bap170 $0^{G 5986}$ (denoted as bap170) also exhibits pupal arrest. Nurf $301^{4}$ contains a splice-donor site mutation that blocks splicing of the fourth intron. The aberrant transcript introduces four additional amino acids (GKLF) and an in-frame stop codon after P1531, truncating C-terminal 609-1230 aa including the essential PHD finger and Bromodomain(Badenhorst et al. 2002). Nurf $301^{4}$ is pupal-lethal with $<60 \%$ pupariation rate(Badenhorst et al. 2005). When transheterozygous with a deletion allele spanning 31 genes including Nurf301, Nurf301 ${ }^{4} / D f(3 L)$ Exel6084 (denoted as nurf301) also shows pupal arrest.

(B) Global chromatin-bound fractions for $\mathrm{GAF}^{\mathrm{L}}$-Halo and $\mathrm{GAF}^{\mathrm{S}}$-Halo in wild type (WT), bap170 and nurf301 mutants. Fast and slow tracking results with propagated errors. All three strains express transgenic $\mathrm{GAF}^{\mathrm{L}}$-Halo or $\mathrm{GAF}^{\mathrm{S}}$-Halo under natural regulation, in the presence of endogenous, untagged GAF. See methods for genotypes of WT, bap170, and nurf301.

(C) Corrected average residence times for $\mathrm{GAF}^{\mathrm{L}}$-Halo and $\mathrm{GAF}^{\mathrm{S}}-\mathrm{Halo}$ in $W T$, bap170 and nurf301 mutants. Error bars represent bootstrapped SD.

(D) GAF ${ }^{\mathrm{L}}$-Halo and $\mathrm{GAF}^{\mathrm{S}}$-Halo distributions on fixed salivary gland polytene nuclei for $W T$, bap170 and nurf 301 mutants. 


\section{Figure 4}

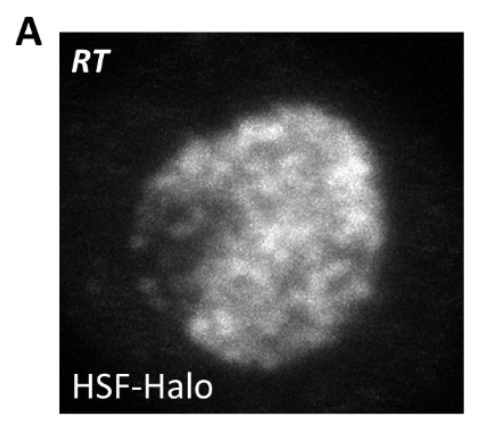

B
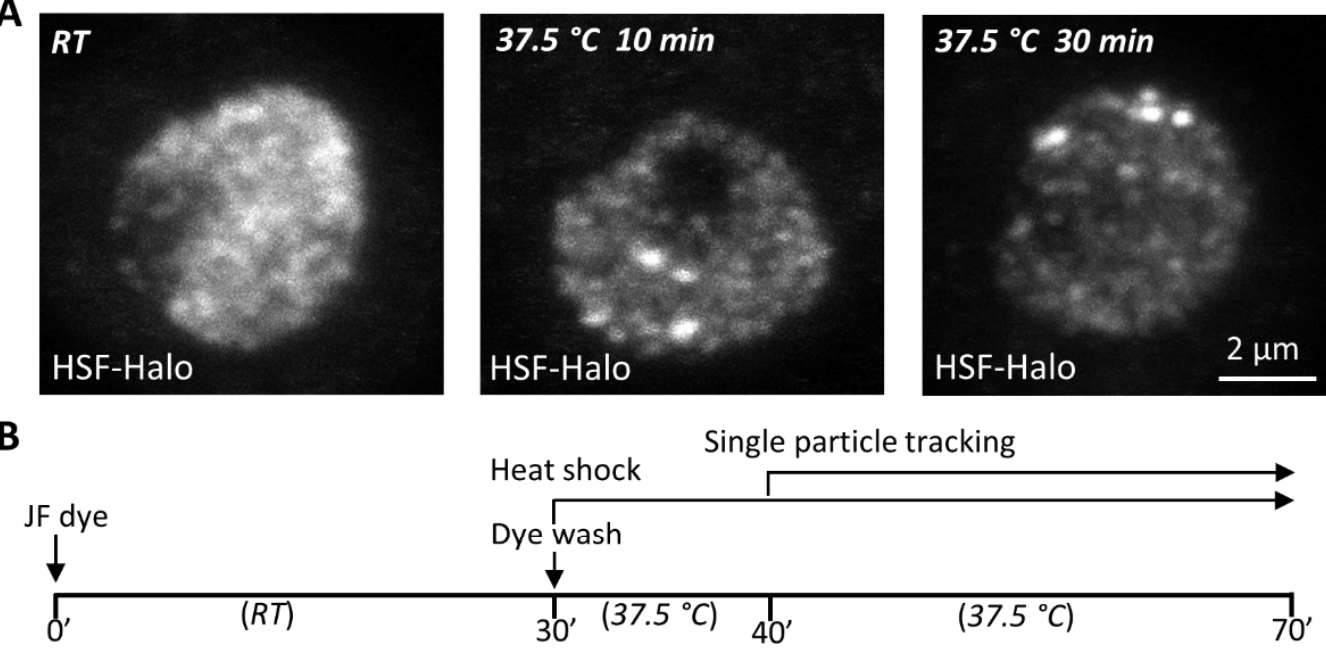

C
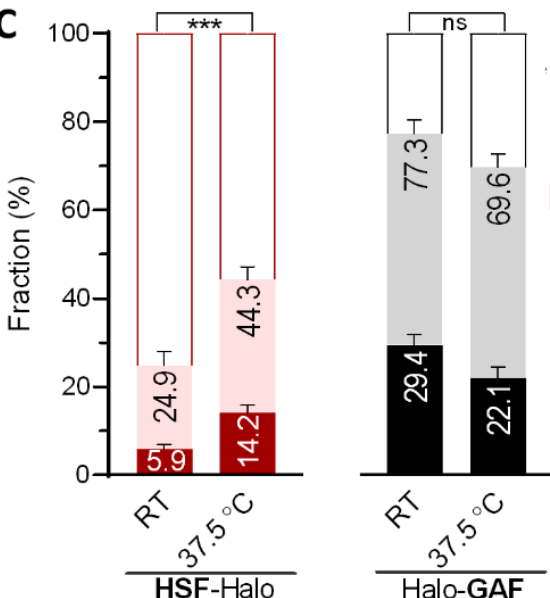

$F_{\text {free }}$

E

10 ms frame (Spot-On)

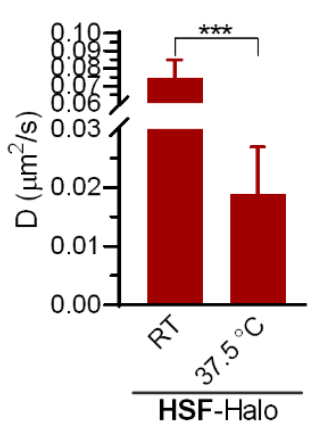

$D_{\text {bound }}$
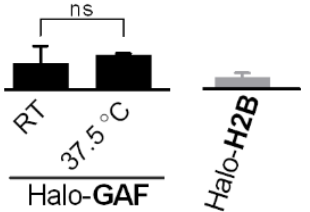

D 2007 Corrected residence time

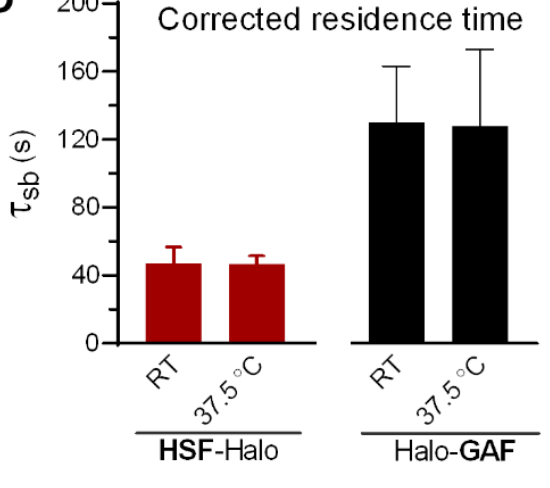

$10 \mathrm{~ms}$ frame (vbSPT) Bound fraction

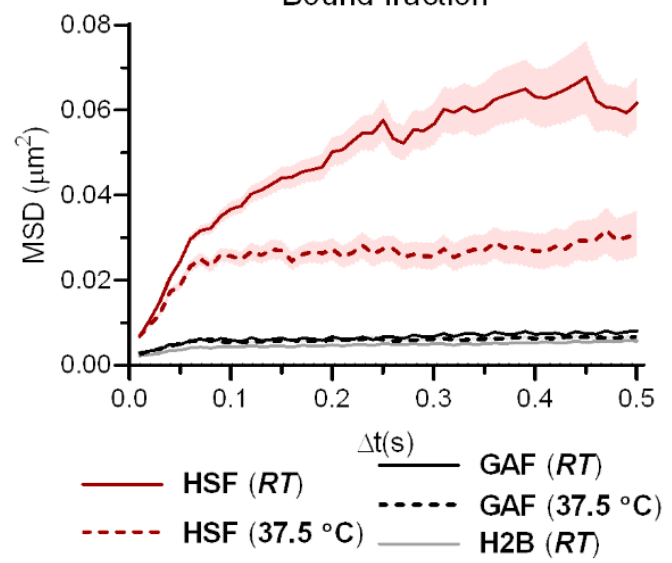

Figure 4. Heat shock increases chromatin binding fraction of HSF without affecting dwell time 
(A) Maximum-intensity z-stack projection of HSF-Halo in fixed hemocytes. HSF-Halo forms several prominent foci upon heat shock at $37.5^{\circ} \mathrm{C}$. Maximum projections of confocal zstacks are shown.

(B) Flow chart of the heat shock and live-hemocyte imaging procedure. SPT starts $10 \mathrm{~min}$ after heat shock and continues over multiple cells (1-2 min per cell) for a total of $30 \mathrm{~min}$ with each sample.

(C) Global chromatin-bound fractions for HSF-Halo and Halo-GAF at room temperature (RT) and $37.5^{\circ} \mathrm{C}$. Fast and slow-tracking results with propagated errors. ***, $p<0.001$, unpaired $t$-test for fast-tracking ( $n=3-4)$.

(D) Corrected average residence times for HSF-Halo and Halo-GAF at $R T$ and $37.5^{\circ} \mathrm{C}$. Error bars represent bootstrapped SD.

(E) Diffusion coefficients derived using Spot-On for bound fractions of HSF-Halo and HaloGAF at $R T$ and $37.5^{\circ} \mathrm{C}$, and Halo-H2B at $R T$. $* * *, p<0.001$, unpaired $t$-test for fasttracking ( $n=3-4)$.

(F) Average MSD (mean $\pm \mathrm{SE}$ ) versus lag time of bound trajectories classified by vbSPT for HSF-Halo, Halo-GAF and Halo-H2B at $R T$ and $37.5^{\circ} \mathrm{C}$, and Halo-H2B at RT. See Fig. S1OA for a zoomed-in section for GAF and $\mathrm{H} 2 \mathrm{~B}$. 


\section{Figure 5}
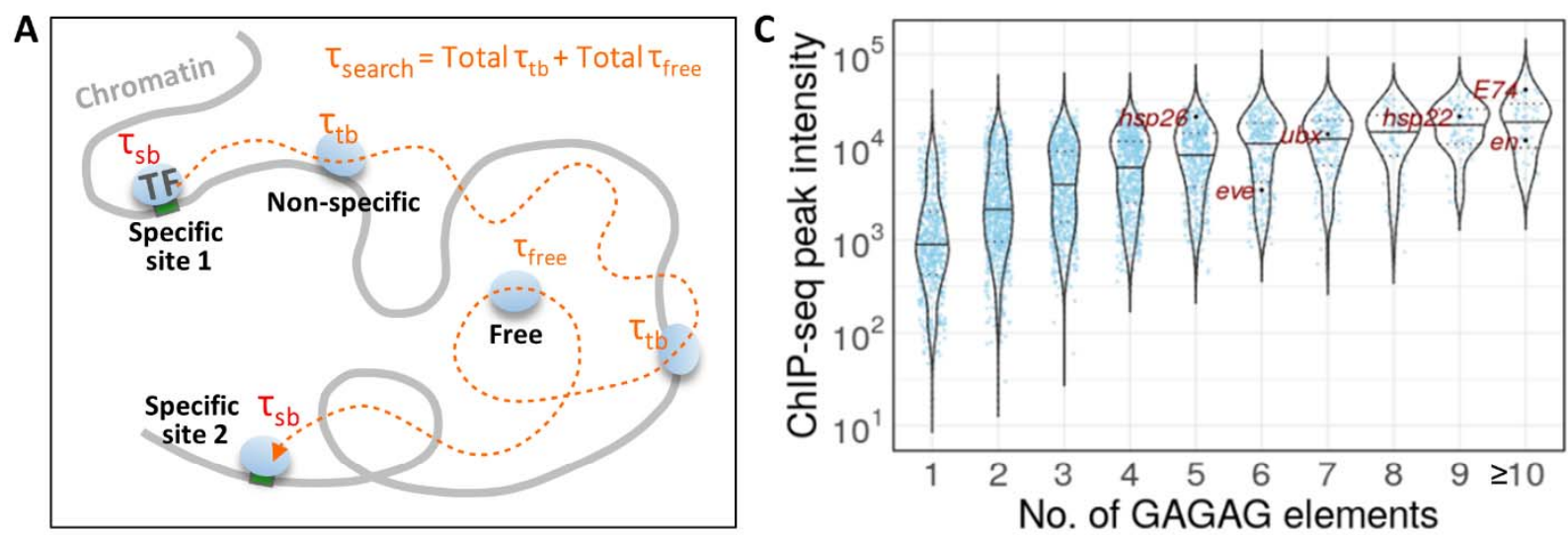

B

\begin{tabular}{c|c|c|c|c|c|c|c|c|c|c}
\hline \multicolumn{1}{c|}{ Factor } & $\mathrm{F}_{\text {bound }}$ & $\mathrm{f}_{\mathrm{sb}}$ & $\tau_{\mathrm{sb}}(\mathrm{s})$ & $\tau_{\text {tb }}(\mathrm{s})$ & $\mathrm{N}_{\text {molecules }}$ & $\mathrm{N}_{\text {sites }}$ & $\mathrm{N}_{\text {trials }}$ & $\tau_{\text {search }}(\mathrm{s})$ & $\mathrm{SI}(\mathrm{s})$ & $\mathrm{O}$ \\
\hline GAF WT & 0.77 & 0.38 & 130.0 & 3.70 & $\begin{array}{c}56,683 \\
\pm 6,025\end{array}$ & $3622 \times 4$ & $23 \pm 8$ & $149.9 \pm 50.1$ & $71.5 \pm 7.6$ & $182 \pm 50 \%$ \\
GAF $\triangle P O Z$ & 0.44 & 0.33 & 42.3 & 2.46 & $\begin{array}{c}44,907 \\
\pm 14,154\end{array}$ & $3622 \times 4$ & $9 \pm 2$ & $104.9 \pm 17.3$ & $47.5 \pm 15.0$ & $89 \pm 30 \%$ \\
GAF $\triangle Q$ & 0.58 & 0.41 & 49.2 & 2.93 & $\begin{array}{c}66,206 \\
\pm 3,064\end{array}$ & $3622 \times 4$ & $8 \pm 2$ & $77.6 \pm 13.0$ & $27.7 \pm 1.3$ & $177 \pm 29 \%$ \\
GAF 37.5 ${ }^{\circ} \mathrm{C}$ & 0.70 & 0.32 & 127.7 & 2.46 & $\begin{array}{c}56,683 \\
\pm 6,025\end{array}$ & $3622 \times 4$ & $40 \pm 16$ & $197.6 \pm 66.5$ & $83.6 \pm 8.8$ & $154 \pm 57 \%$ \\
HSF 37.5 ${ }^{\circ} \mathrm{C}$ & 0.44 & 0.32 & 46.7 & 2.87 & $\begin{array}{c}9,543 \\
\pm 613\end{array}$ & $442 \times 4$ & $9 \pm 2$ & $118.5 \pm 20.7$ & $30.6 \pm 2.0$ & $153 \pm 21 \%$ \\
\hline
\end{tabular}

Figure 5. High site occupancy and remodeler autonomy quantifies pioneering criteria

(A) Schematic of a TF trajectory between two specific chromatin targets showing the search time $\tau_{\text {search }}$, stable $\tau_{\text {sb }}$ and transient $\tau_{\text {tb }}$ dwell times, and $\tau_{\text {free. }}$. The TF molecule dissociates from a specific target, and samples nonspecific sites for a number of trials before encountering the next specific target. The tsearch equation is indicated (see methods).

(B) Violin plots of GAF ChIP-seq peak intensities (analysis of S3 Table from Fuda et al, 2015) in hemocyte-like $S 2$ cells plotted by the number of non-overlapping GAGAG elements identified with HOMER ${ }^{96}$.

(C) Key SPT and $\mathrm{N}_{\text {molecules }}$ parameters measured in this study and $\mathrm{N}_{\text {sites }}$ from the literature ${ }^{17,48}$ are used to calculate occupancy levels for GAF and HSF. 


\section{Figure 6}
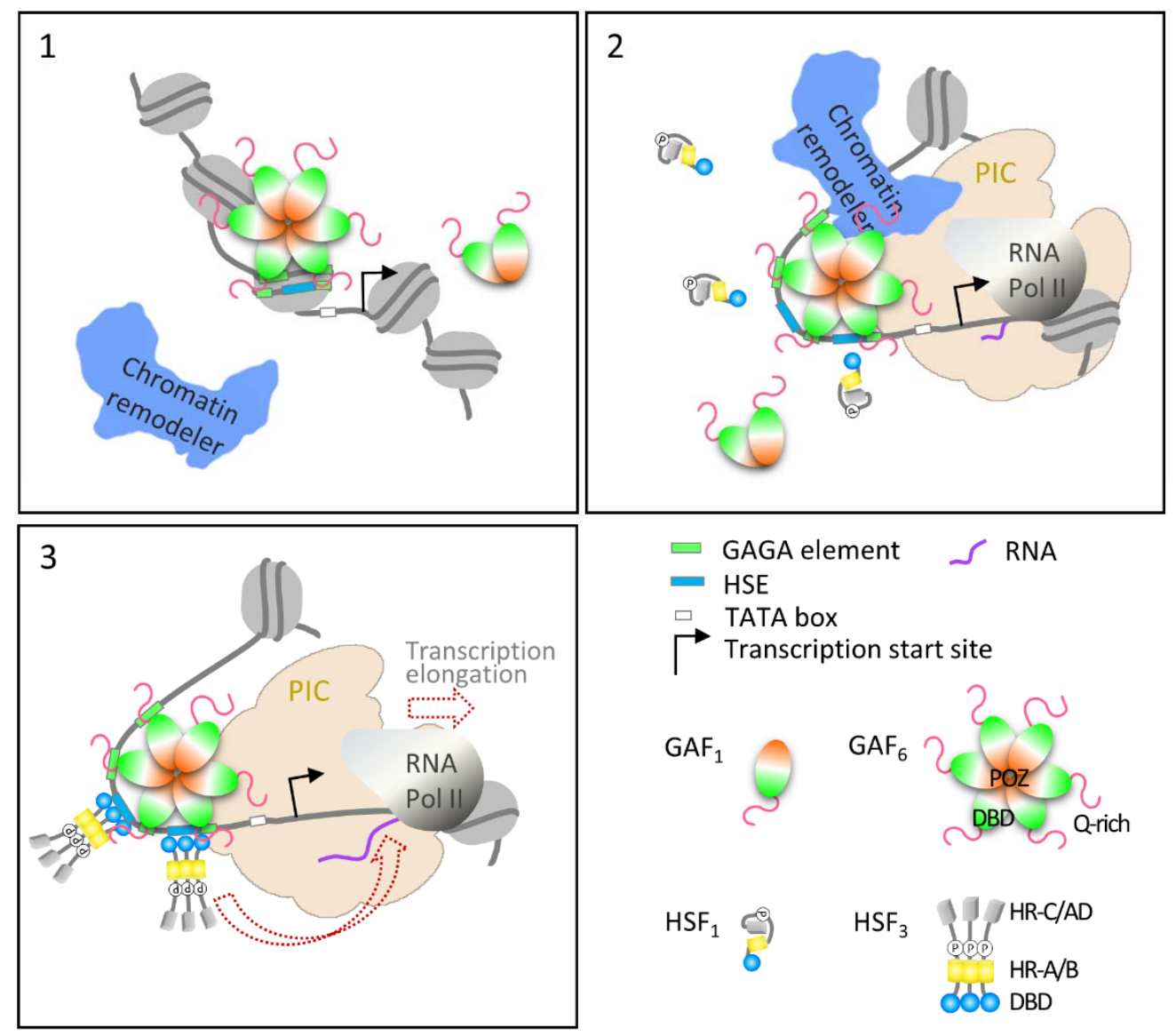

$\square$ GAGA element $\sim$ RNA

- HSE

․ TATA box

$\longrightarrow$ Transcription start site
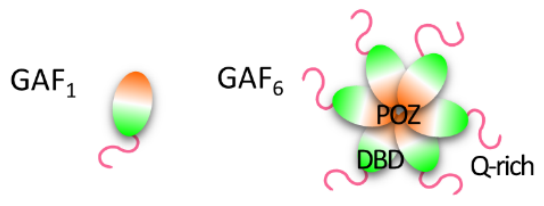

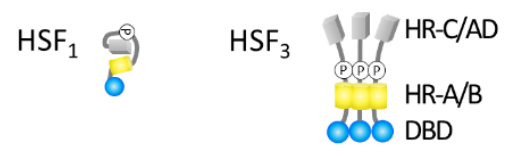

Figure 6. Pioneering of chromatin accessibility is a process involving multiple inputs

Model: GAF binds autonomously to nucleosomal sites at the first stage of pioneering (box 1). High GAF occupancy at a Hsp promoter with clustered GAGA elements maintains chromatin accessibility for neighboring factor HSF and assembly of the preinitiation complex and paused RNA Pol II (box 2). The substantially constrained diffusivity ( $D_{\text {bound }}$ ) of stably bound GAF may reflect multisite interactions of a GAF multimer with clustered GAGA elements (Katsani et al, 1999), locking down GAF with prolonged residence time. HSF trimers bind to accessible chromatin DNA with high affinity on heat shock to trigger RNA Pol II elongation (box 3). See discussion for details. 
bioRxiv preprint doi: https://doi org/10.1101/2021.10.21.465351; this version posted January 7, 2022. The copyright holder for this preprint (which was not certified by peer review) is the author/funder, who has granted bioRxiv a license to display the preprint in perpetuity. It is made available under aCC-BY-NC-ND 4.0 International license.

Figure S1
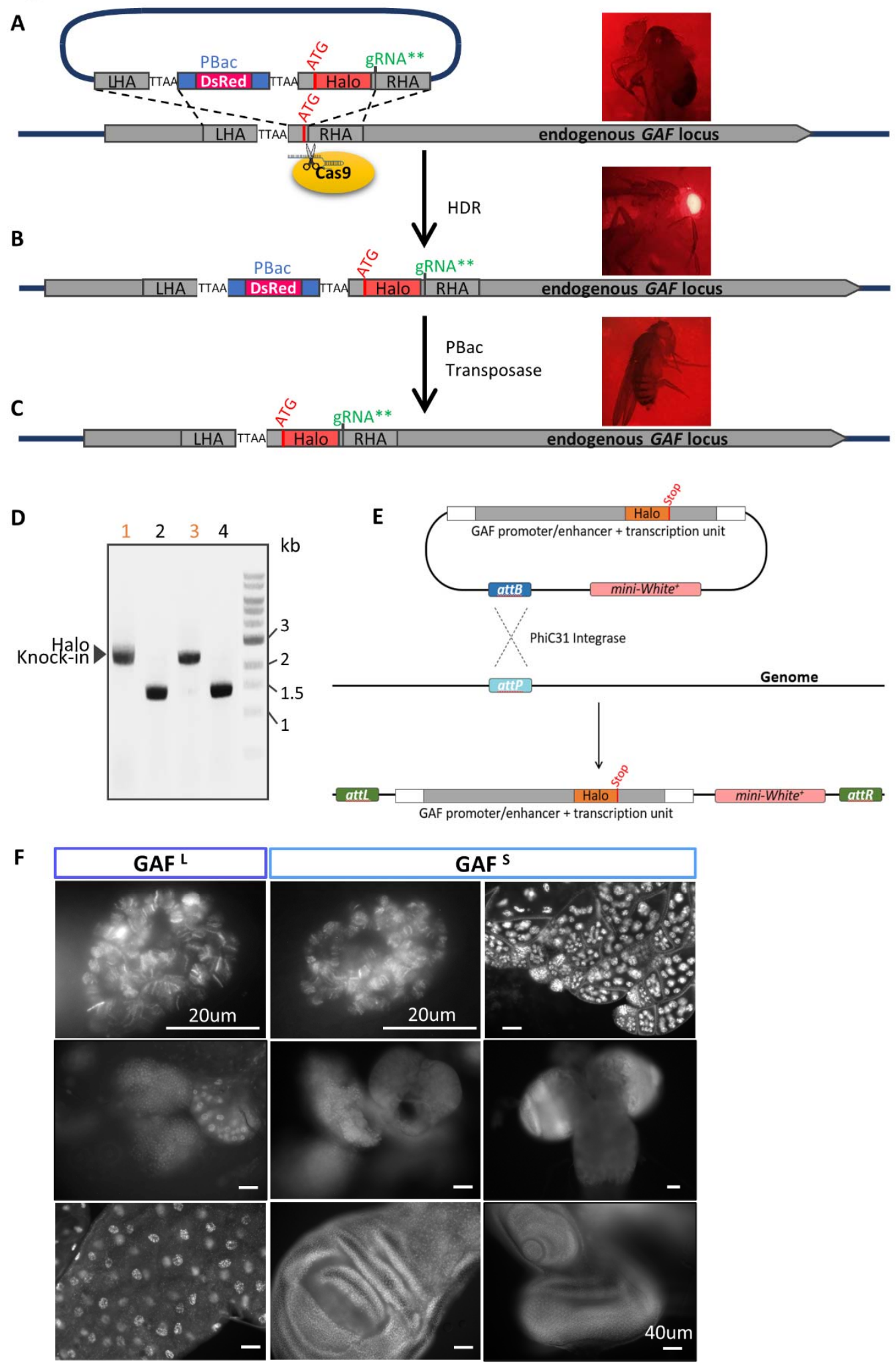


\section{Figure S1. Generation of N-terminal Halo-GAF knock-in fly strain and C-terminal GAF-Halo} transgenic fly strains.

(A) Donor plasmid design for homology directed repair (HDR). HaloTag and a flexible linker (GGSGS, not shown) are placed downstream of the start codon ATG. A PBac transposon containing a DsRed cassette is inserted into a nearby genomic TTAA site adjacent to the gRNA target site in the coding region that is close to the start codon ATG. The TTAA site is duplicated so that both ends of the PBac transposon contain a TTAA sequence. Approximately $1 \mathrm{~kb}$ fragment downstream of the gRNA target site is cloned as the right homology arm (RHA), with silent mutations (gRNA**) introduced to destroy the gRNA PAM sequence in the donor plasmid. Similarly, a $1 \mathrm{~kb}$ fragment upstream of the genomic TTAA site is cloned as the left homology arm (LHA).

(B) LHA and RHA mediate HDR upon Cas9 cleavage, inserting HaloTag along with the DsRed cassette. Flies that have undergone HDR can be identified by eye DsRed fluorescence.

(C) By crossing to a fly strain expressing PBac transposase, the DsRed cassette can be removed, as indicated by loss of fluorescence, leaving only one TTAA sequence, thereby allowing scarless HaloTag knock-in with a removable selection marker. Arrows indicate positions of the primers used for validating HaloTag insertion.

(D) PCR validation of HaloTag knock-in after DsRed cassette removal (lane 1 and 3). HaloGAF homozygous flies are viable, showing only 1 band $~ 900$ bp larger than flies without HaloTag knock-in (lane 2 and 4).

(E) Strategy used to generate transgenic fly strains $\mathrm{GAF}^{\mathrm{L}}$-Halo, $\mathrm{GAF}^{\mathrm{S}}$-Halo, Halo-H2B and HSF-Halo. An $\sim 15 \mathrm{~kb}$ fragment containing the Trl transcription unit and $\sim 1 \mathrm{~kb}$ upstream and downstream regions was cloned, and HaloTag ORF was inserted upstream of the stop codons for $\mathrm{GAF}^{\mathrm{L}}$ or $\mathrm{GAF}^{\mathrm{S}}$, respectively. Thus, each of the two transgenic flies express a Halo-tagged GAFL or GAFS isoform and another isoform (non-tagged), under native Trl promoter control.

(F) Tissue-specific expression of transgenic $G A F^{L}-H a l o$ and $G A F^{S}-H a l o$. Shown are major expressing larval tissues. $\mathrm{GAF}^{\mathrm{L}}$-Halo: salivary gland, lymph gland, intestine; $\mathrm{GAF}^{\mathrm{S}}$-Halo: salivary gland, lymph gland, wing disc, ovary, brain, eye-antenna disc. 
bioRxiv preprint doi: https://doi.org/10.1101/2021.10.21.465351; this version posted January 7, 2022. The copyright holder for this preprint (which was not certified by peer review) is the author/funder, who has granted bioRxiv a license to display the preprint in perpetuity. It is made available under aCC-BY-NC-ND 4.0 International license.

\section{Figure S2}

A $3^{\text {rd }}$ instar larvae

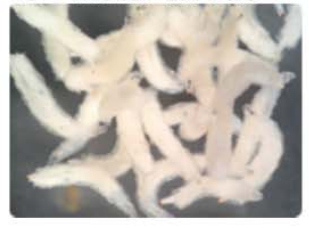

B Halo-GAF (knock-in)
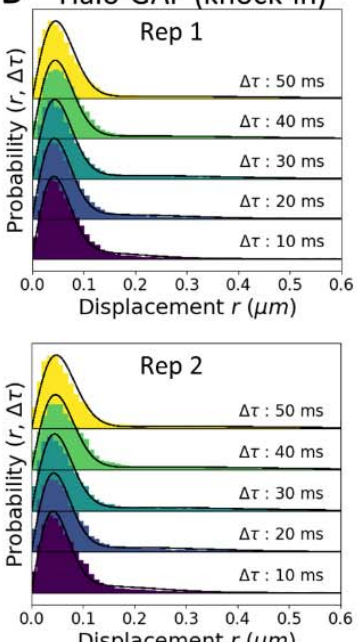

Displacement $r(\mu m)$

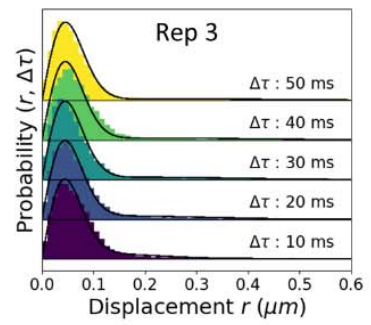

C

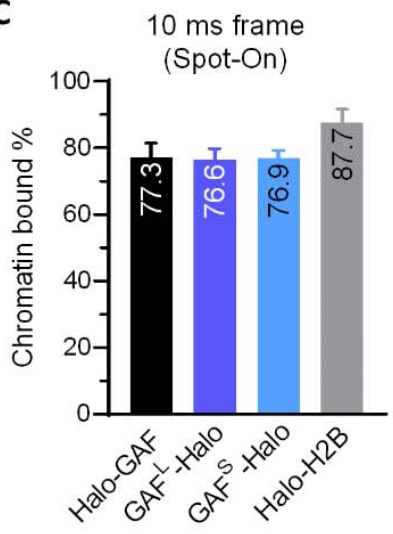

Dissection

JF dye labeling $(0.5 \mathrm{~h})$

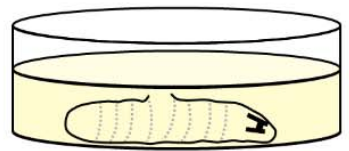

Wash

Coverglass bottom
Imaging (1 2 h)

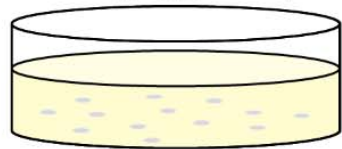

Coverglass bottom
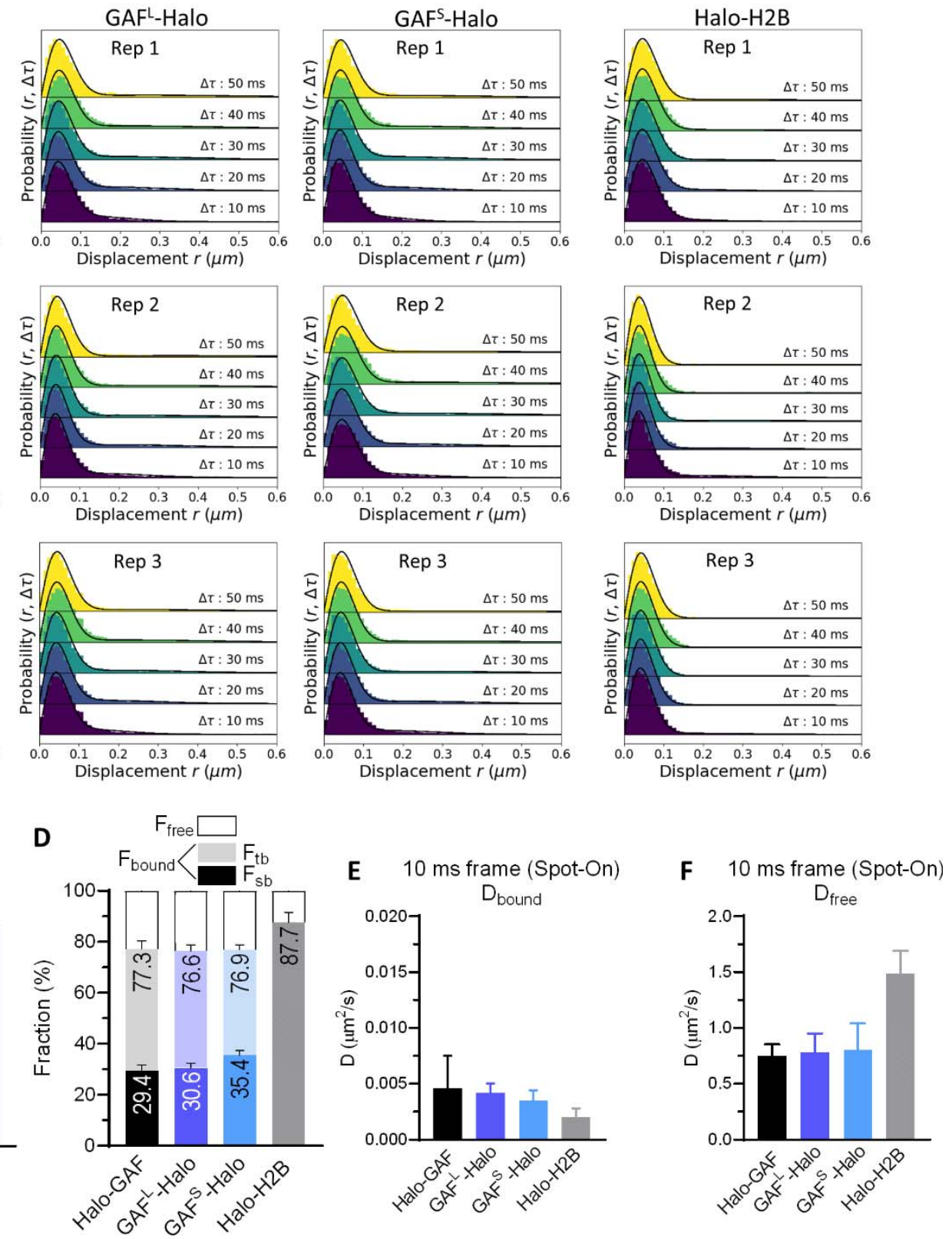

Figure S2. Hemocyte imaging and fast-tracking diffusive parameters for Halo-GAF, GAF ${ }^{\mathrm{L}}$-Halo, 
1073

1074

1075

1076

1077

1078

1079

1080

1081

1082

1083

1084

1085

1086

1087

1088

1089

1090

(A) Experimental timeline of single-particle imaging with 3rd instar larval hemocytes. 3rd instar larvae are washed with DI H2O (left) and dissected in a coverglass bottom dish containing Schneider's medium and JF dye at room temperature. Upon dissection hemocytes are released into the medium and labeled for $30 \mathrm{~min}$, the rest of the larval tissues are discarded (middle). Cells are briefly washed twice with fresh media and imaged for 1-2 $\mathrm{h}$.

(B) Spot-On fits of Halo-GAF, GAF ${ }^{\mathrm{L}}$-Halo, $\mathrm{GAF}^{\mathrm{S}}-\mathrm{HalO}$, Halo-H2B fast-tracking data.

(C) Spot-On kinetic modeling of fast-tracking data shows $77 \%$ of Halo-GAF is chromatin bound. Similar values are obtained for isoforms $G A F^{L}$ and $G A F^{S}$ individually tagged in the presence of untagged GAF isoforms. Results are mean \pm SD from three biological replicates.

(D) Chromatin-free fraction $\left(F_{\text {free }}\right)$, long- and short-lived chromatin-binding fractions $\left(F_{s b}\right.$ and $F_{t b}$ ) of HaloTagged GAF fusions extracted from fast- and slow-tracking data in (C) and (Fig. S3E), respectively, with error propagation.

(E) Diffusion coefficients of bound fraction ( $D_{\text {bound }}$ ) for Halo-GAF, GAF - Halo, $G A F^{S}$-Halo, Halo-H2B derived by Spot-On.

(F) Diffusion coefficients of free fraction (Dfree) for Halo-GAF, GAF ${ }^{L}$-Halo, $G A F^{S}$-Halo, HaloH2B derived by Spot-On. 
bioRxiv preprint doi: https://doi.org/10.1101/2021.10.21.465351; this version posted January 7, 2022. The copyright holder for this preprint (which was not certified by peer review) is the author/funder, who has granted bioRxiv a license to display the preprint in perpetuity. It is made available under aCC-BY-NC-ND 4.0 International license.

\section{Figure S3}

A

Slow tracking: $500 \mathrm{~ms}$

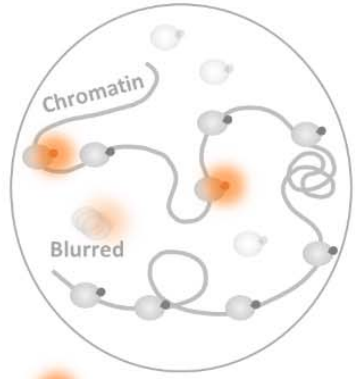

JF552 labeled factor

- JF700 labeled factor
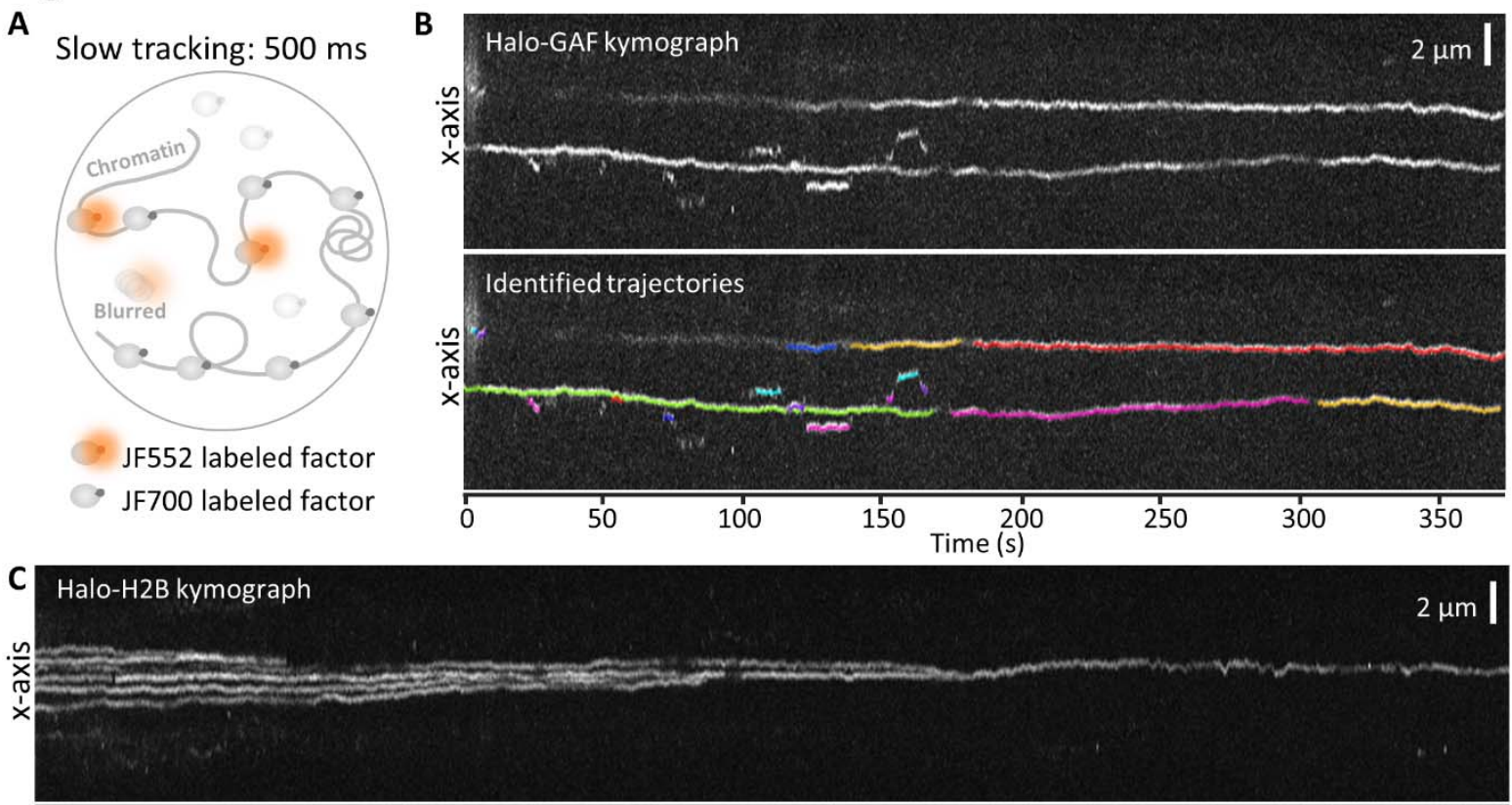

Identified trajectories
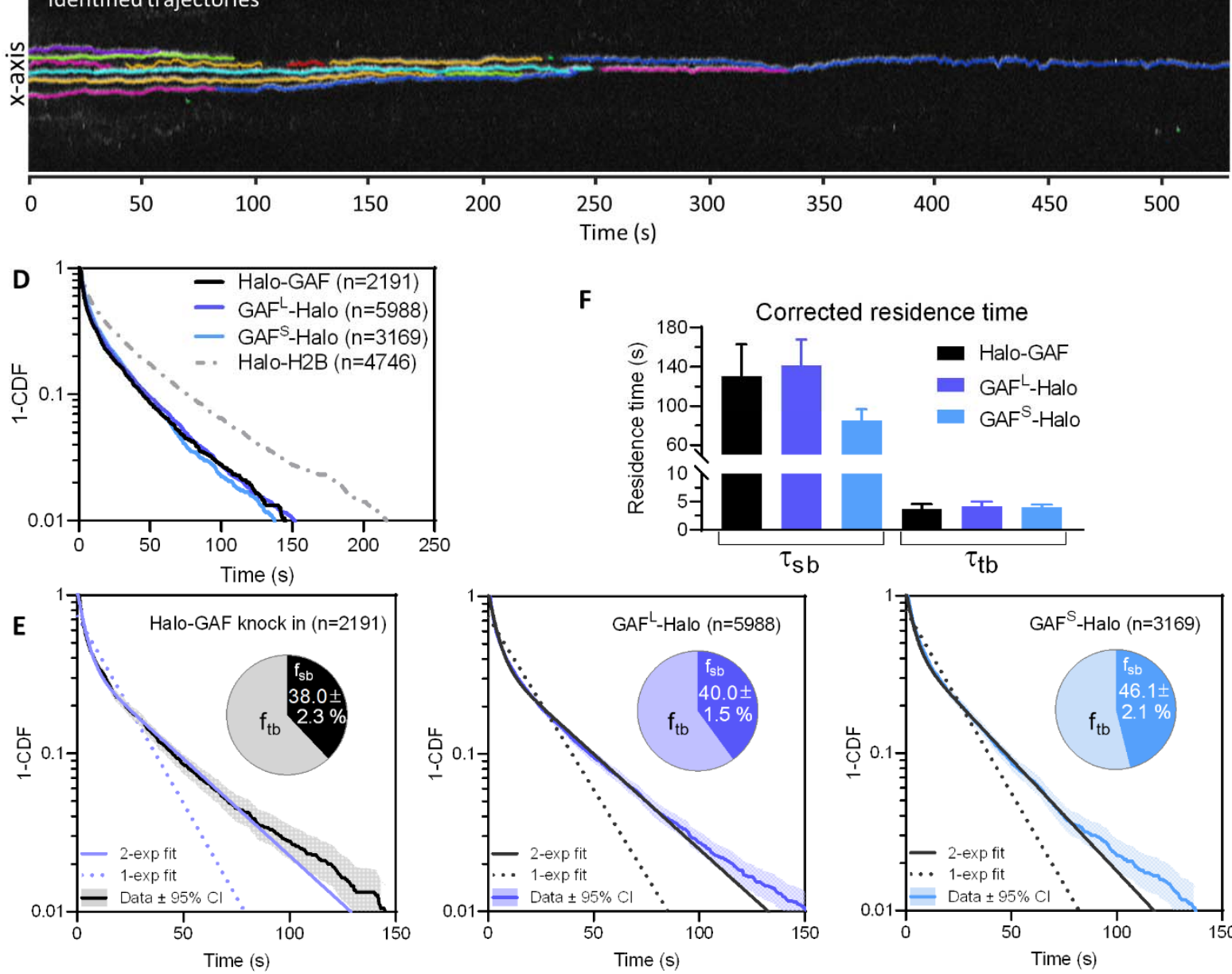
(A) Fast and slow tracking regimes. Fast tracking with $10 \mathrm{~ms}$ frame rate and high laser power allows single molecule imaging to distinguish slow (chromatin-bound) and fast (chromatin-free) diffusing subpopulations. Slow tracking uses low-intensity excitation and 500 ms exposure time to motion blur diffusing molecules and selectively observe the dwell times of chromatin-bound molecules. A higher concentration of JF700 is added to block labelling of most HaloTag protein fusions, while a much lower concentration of JF552 is used to sparsely label a small fraction of HaloTag so that each nucleus shows only $2 \sim 10$ molecules per frame during image acquisition.

(B) Kymograph of a Halo-GAF slow tracking movie shows traces of bound GAF molecules over time (upper). Trajectories identified from the raw movie are plotted on the kymograph using separate colors (lower).

(C) Kymograph of a Halo-H2B slow tracking movie shows traces of bound $\mathrm{H} 2 \mathrm{~B}$ molecules over time (upper). Trajectories identified from the raw movie are plotted on the kymograph (lower).

(D) Survival probability curves (1-CDF) plotted from apparent dwell times of thousands ( $n$ ) of single-molecule chromatin-binding events for Halo-GAF, GAFL-Halo and GAFS-Halo.

(E) One-component and two-component exponential fit of survival probabilities (1-CDF) from slow tracking data of Halo-GAF, GAF - Halo and $G A F^{S}-$ Halo. Pie charts show the stable-binding $\left(f_{s b}\right)$ and transient-binding $\left(f_{s b}\right)$ fractions derived from two-component fits, and errors represent bootstrapped SD.

(F) Corrected average residence times for stable- $\left(\tau_{\mathrm{sb}}\right)$ and transient- $\left(\tau_{\mathrm{tb}}\right)$ binding by transgenic $\mathrm{GAF}^{\mathrm{L}}$-Halo and $\mathrm{GAF}^{\mathrm{S}}$-Halo. 


\section{Figure S4}

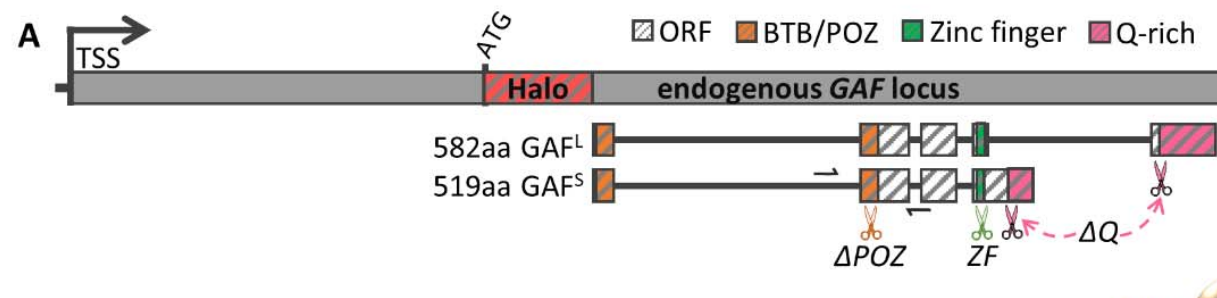

B
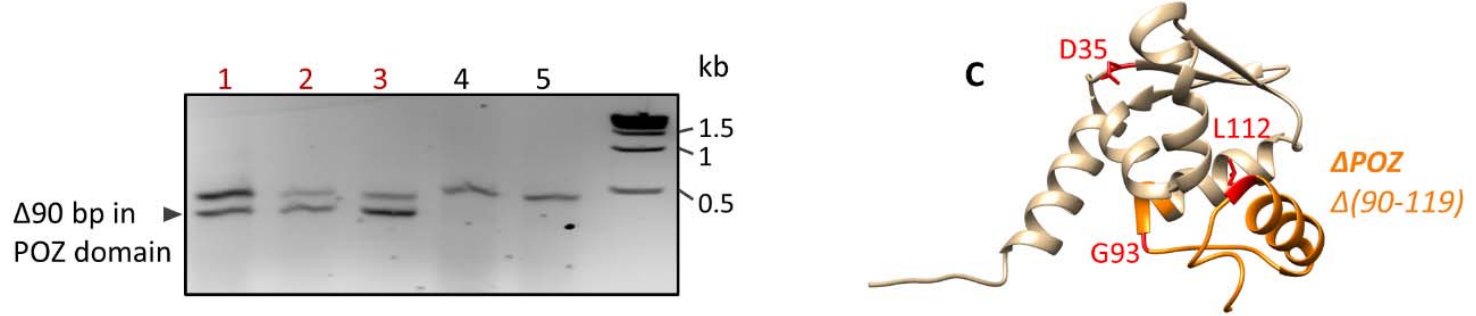

D

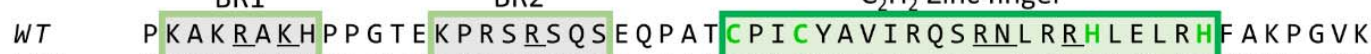

$Z F^{9}$ PKAKRAKHPPGTEKPRSRSQSEQPATCPICYAVIRQS - - LRRHLELRHFAKPGVK

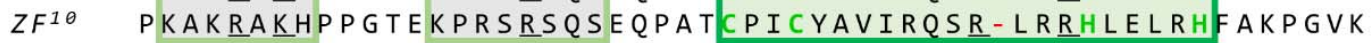

$\mathbf{E}$

WT Long-isoform Q-domain (85 Q/179 AA)

WT Short-isoform Q-domain (31 Q/79 AA)

$$
\begin{aligned}
& \text { QGVLPQQQQQQQLQQQHQQHLTATLAGGGQAYIKHEGGGGGGTGQQQQQQAAQQQGMQNVIHI } \\
& 441 \\
& \begin{array}{l}
\text { VGDQVFIPQQQQPQPQ* } \\
504 \\
519
\end{array}
\end{aligned}
$$

$\triangle Q$ double frame shift

7 bp deletion in Long-isoform leads to frame shift (179 AA $\rightarrow 39$ AA)

RSRRFNPCTLYRVCKSRRIRMPSNSSSSSSSNNNNNSSKQ*
403

$7 \mathrm{bp}$ deletion in Short-isoform leads to frame shift (79 AA $\rightarrow 19$ AA)

AVCCPSSSNSSNCSSNTSST*

440

458

Figure S4. Generation of mutations in functional domains of Halo-GAF by CRISPR/Cas9 gene editing.

(A) In the Halo-GAF fly strain, Cas9 and gRNA were introduced to target the BTB/POZ domain, zinc finger, and Q-rich domains, respectively. The BTB/POZ domain is separated by a large intron. A gRNA target site in the second exon (orange scissors) was selected and a donor plasmid containing a $90 \mathrm{bp}$ deletion ( $\triangle P O Z$ ) was constructed for homologydirected repair (HDR). For zinc finger mutations, we selected a gRNA target site in the 
zinc finger coding region (green scissors), and screened for in-frame small deletions generated by non-homologous end joining (NHEJ). To generate deletions of both Q-rich domains in long and short isoforms $(\Delta Q)$, two gRNAs targeting the upstream ends of two Q-rich domains (pink scissors) were introduced at the same time, and we screened for double frame-shift deletions induced by NHEJ. After gRNA and donor plasmid injection, fly progeny were crossed with balancer chromosome, candidates homozygous-lethal or with reduced viabilities were verified by PCR and sanger sequencing. Half arrows indicate positions of the PCR primers used in (B). TSS, transcription start site.

(B) PCR validation of $\triangle P O Z$. Lanes 1-3 show two PCR bands indicating precise deletion in one allele; lanes 4-5 are two lines without the precise deletion. Sanger sequencing verified a precise $90 \mathrm{bp}$ deletion in one allele.

(C) POZ domain model structure. 90bp deletion in the second exon generates a 30-AA deletion ( $\triangle 90-119$ ) of the POZ domain ( $\triangle P O Z$, orange), which includes G93 and L112 (red) that have been shown to be essential for transcription activation ${ }^{29}$.

(D) Amino acid sequence of GAF DNA binding domain, which contains a single $\mathrm{C} 2 \mathrm{H} 2$ zinc finger (green rectangle) and two upstream basic regions (BR1 and BR2, yellow rectangle). Amino acids involved in recognizing the GAGAG consensus sequence are underlined ${ }^{108}$. Two zinc finger mutations were isolated and verified by sanger sequencing, $Z F^{9}$ and $Z F^{10}$, with R356 and N357, or R356 deleted, respectively.

(E) Amino acid sequence of GAF Q-rich domains for long and short isoforms. In $\Delta Q$, a $7 \mathrm{bp}$ deletion was identified by sanger sequencing in both isoforms at the upstream ends of Q-rich domains, resulting in frameshifts and truncations of the Q-rich domains from both isoforms. P403 in the long isoform and A440 in the short isoform are deleted and the subsequent amino acids are newly introduced by the frame shifts. 
bioRxiv preprint doi: https://doi.org/10.1101/2021.10.21.465351; this version posted January 7, 2022. The copyright holder for this preprint (which was not certified by peer review) is the author/funder, who has granted bioRxiv a license to display the preprint in perpetuity. It is made available under aCC-BY-NC-ND 4.0 International license.

\section{Figure S5}

A

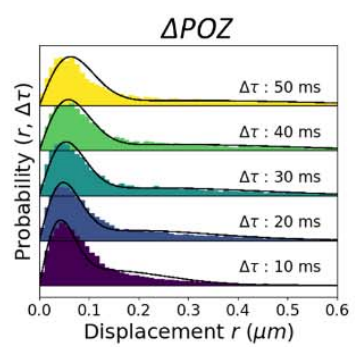

Halo-GAF $10 \mathrm{~ms}$ frame rate displacement histograms and 2-state Spot-On fits
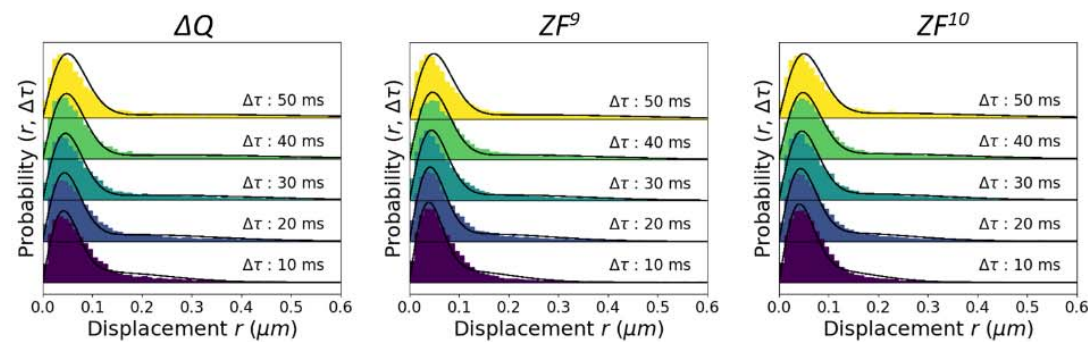

B

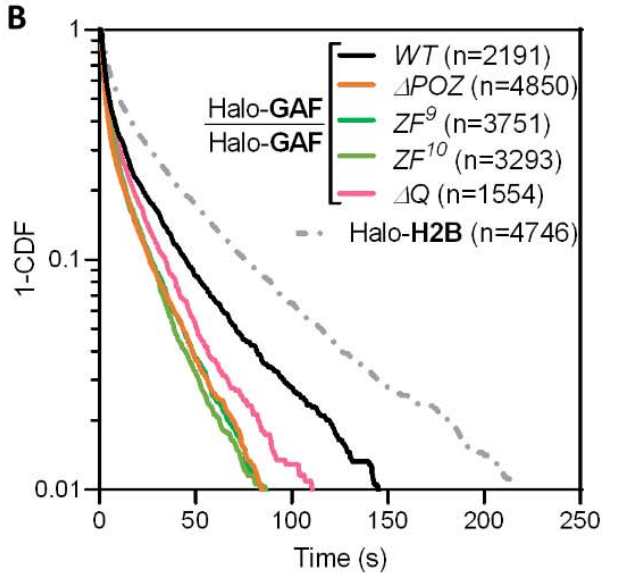

D

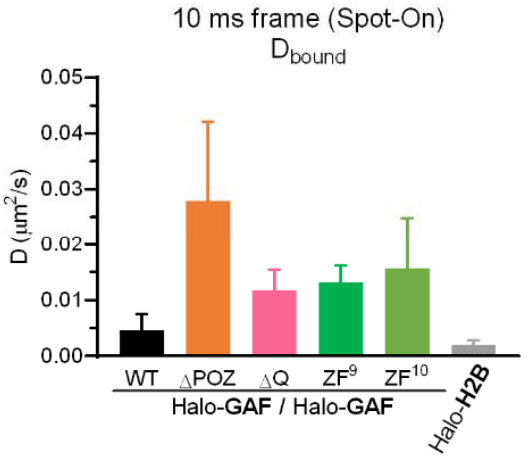

$\mathbf{E}$

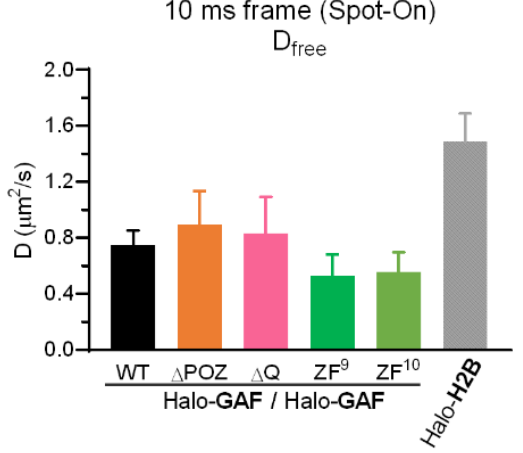

C Halo-GAF $500 \mathrm{~ms}$ frame rate 1-CDF and exponential decay fits
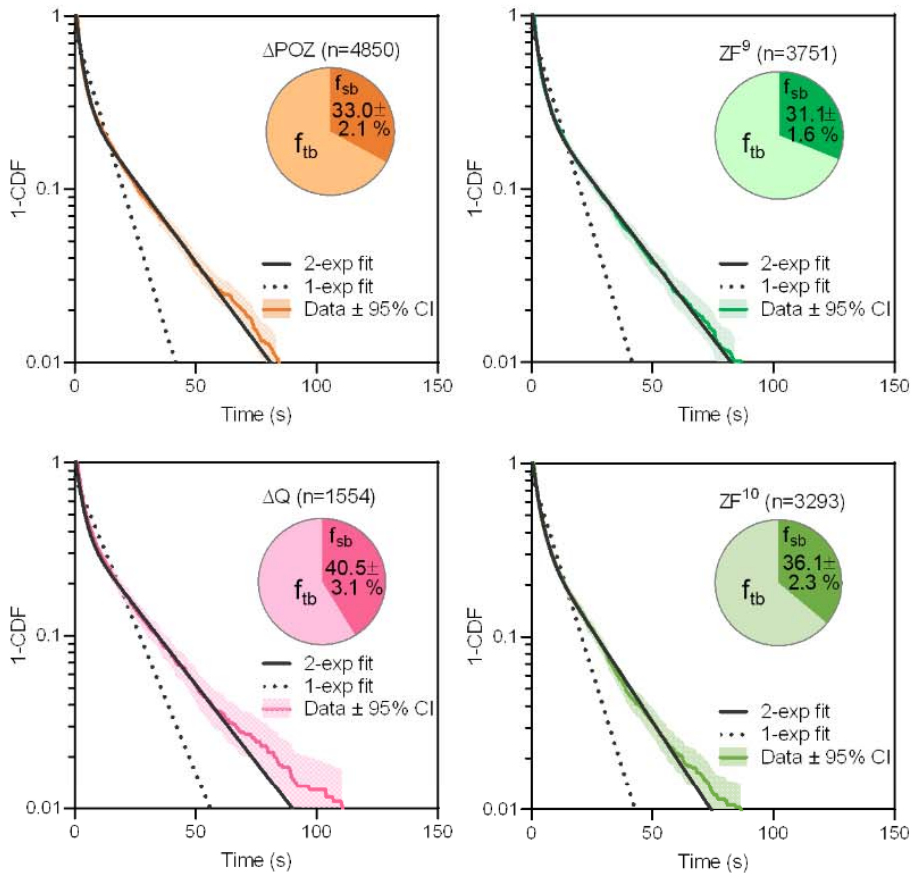

$\mathbf{F}$

$500 \mathrm{~ms}$ frame

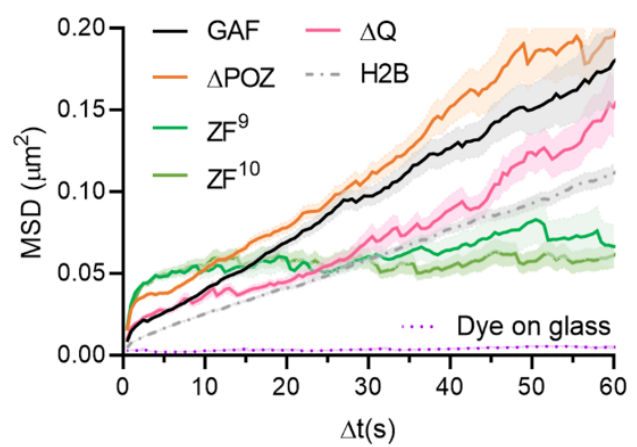


(A) Spot-On fits of fast-tracking data for Halo-GAF mutants (see Fig. S2B for WT).

(B) Survival probability curves (1-CDF) from apparent dwell times of $>1000$ single-molecule chromatin-binding events, for WT and mutant Halo-GAF.

(C) One-component and two-component exponential fit of survival probabilities (1-CDF) from slow tracking data of Halo-GAF mutants (see Fig. S3E for WT). Pie charts show the stable-binding $\left(f_{s b}\right)$ and transient-binding $\left(f_{s b}\right)$ fractions derived from two-component fits.

(D) Diffusion coefficients of bound fraction $\left(D_{\text {bound }}\right)$ for Halo-GAF and Halo-H2B derived by Spot-On.

(E) Diffusion coefficients of free fraction $\left(D_{\text {free }}\right)$ for Halo-GAF and Halo-H2B derived by SpotOn.

(F) Average MSD versus lag time for WT and Halo-GAF mutants at 500 ms frame rate. Mean and SE (shaded) are shown. System noise is shown by the MSD of dye molecules stuck on coverglass. 
bioRxiv preprint doi: https://doi.org/10.1101/2021.10.21.465351; this version posted January 7, 2022. The copyright holder for this preprint (which was not certified by peer review) is the author/funder, who has granted bioRxiv a license to display the preprint in perpetuity. It is made available under aCC-BY-NC-ND 4.0 International license.

\section{Figure S6}

A

GAF-Halo $10 \mathrm{~ms}$ frame rate displacement histograms and 2-state Spot-On fits
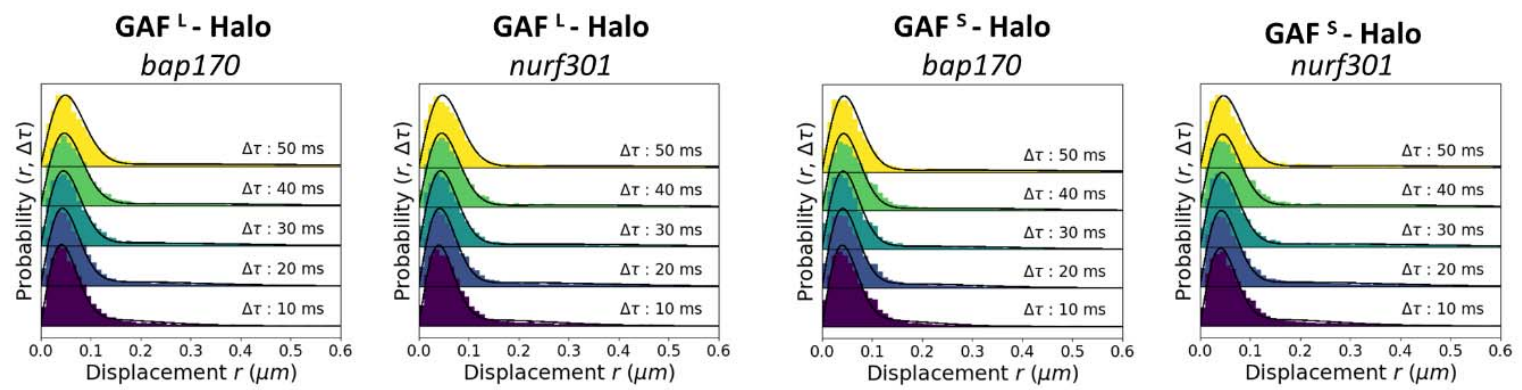

B

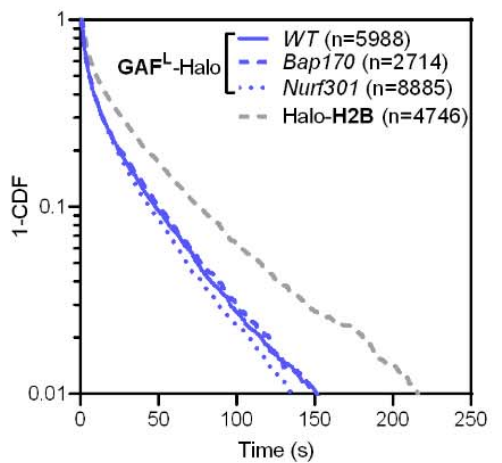

C

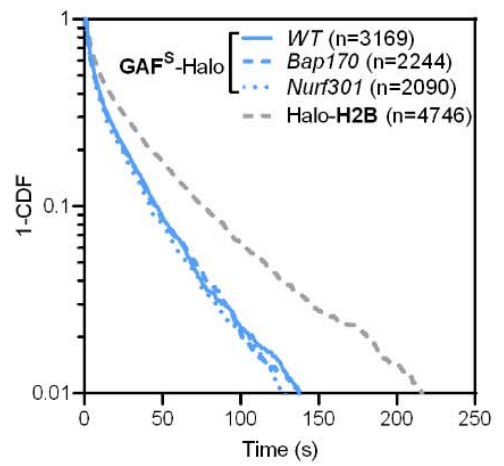

$\mathbf{E}$

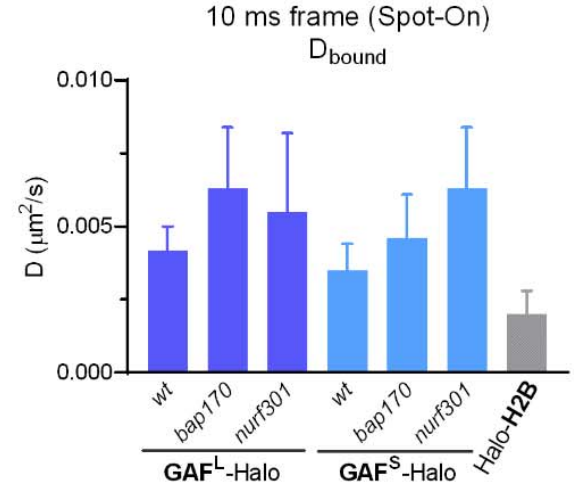

D

GAF-Halo 500 ms frame rate 1-CDF and exponential decay fits
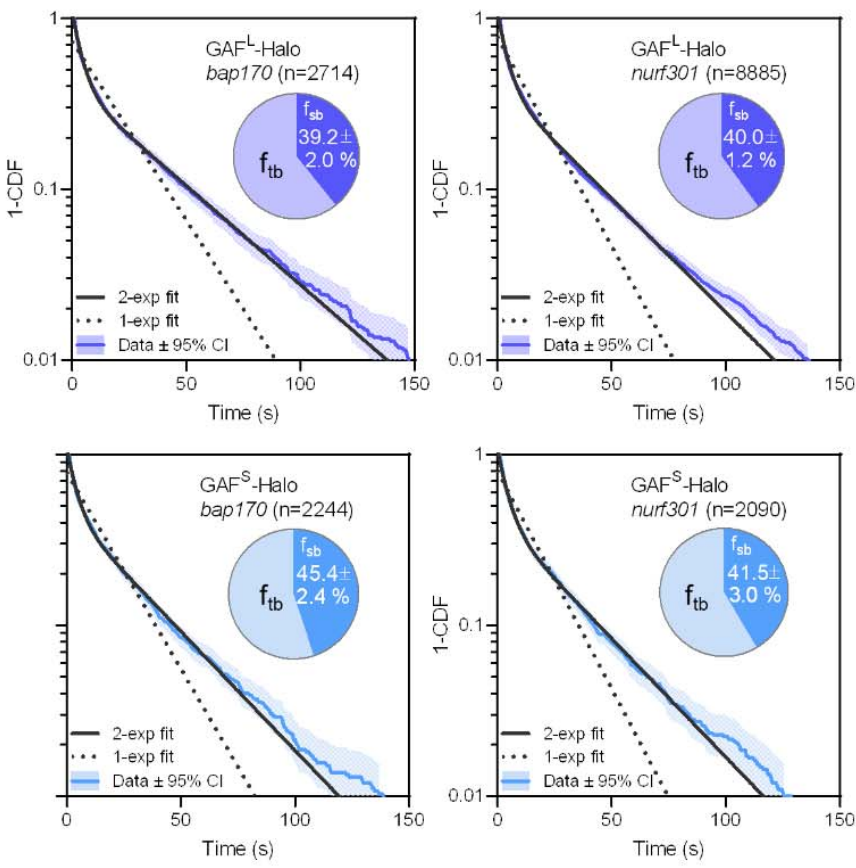

$\mathbf{F}$ 10 ms frame (Spot-On)

$D_{\text {free }}$

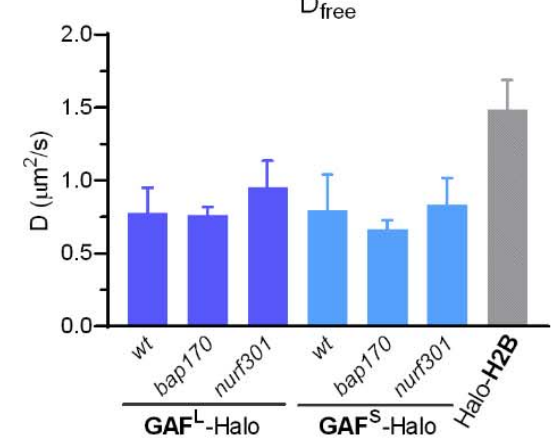


(A) Spot-On fits of fast-tracking data for $\mathrm{GAF}^{\mathrm{L}}$-Halo and $\mathrm{GAF}^{\mathrm{S}}-\mathrm{Halo}$ in bap170 and nurf301 mutants (see Fig. S2B for WT). See methods for genotypes of WT, bap170, and nurf 301.

(B) Survival probability curves (1-CDF) from apparent dwell times of more $>1000$ singlemolecule chromatin-binding events for $\mathrm{GAF}^{\mathrm{L}}$-Halo in $W T$, bap170 and nurf301 mutants.

(C) Survival probability curves (1-CDF) from apparent dwell times of more $>1000$ singlemolecule chromatin-binding events for $\mathrm{GAF}^{\mathrm{S}}$-Halo in $W T$, bap170 and nurf301 mutants.

(D) One-component and two-component exponential fit of survival probabilities (1-CDF) from slow tracking data for $\mathrm{GAF}^{\mathrm{L}}-\mathrm{Halo}$ and $\mathrm{GAF}^{\mathrm{S}}-\mathrm{Halo}$ in bap170 and nurf301 mutants (see Fig. S3D for $W T$ ). Pie charts show the stable-binding $\left(f_{s b}\right)$ and transient-binding $\left(f_{s b}\right)$ fractions derived from two-component fits.

(E) Diffusion coefficients of bound fraction $\left(D_{\text {bound }}\right)$ for $G A F^{L}-H a l o, G A F^{S}-H a l o$ and Halo-H2B derived by Spot-On.

(F) Diffusion coefficients of free fraction $\left(D_{\text {free }}\right)$ for $G A F^{L}-H a l o, G A F^{S}-$ Halo and Halo-H2B derived by Spot-On. 
bioRxiv preprint doi: https://doi.org/10.1101/2021.10.21.465351; this version posted January 7, 2022. The copyright holder for this preprint (which was not certified by peer review) is the author/funder, who has granted bioRxiv a license to display the preprint in perpetuity. It is made available under aCC-BY-NC-ND 4.0 International license.

\section{Figure S7}

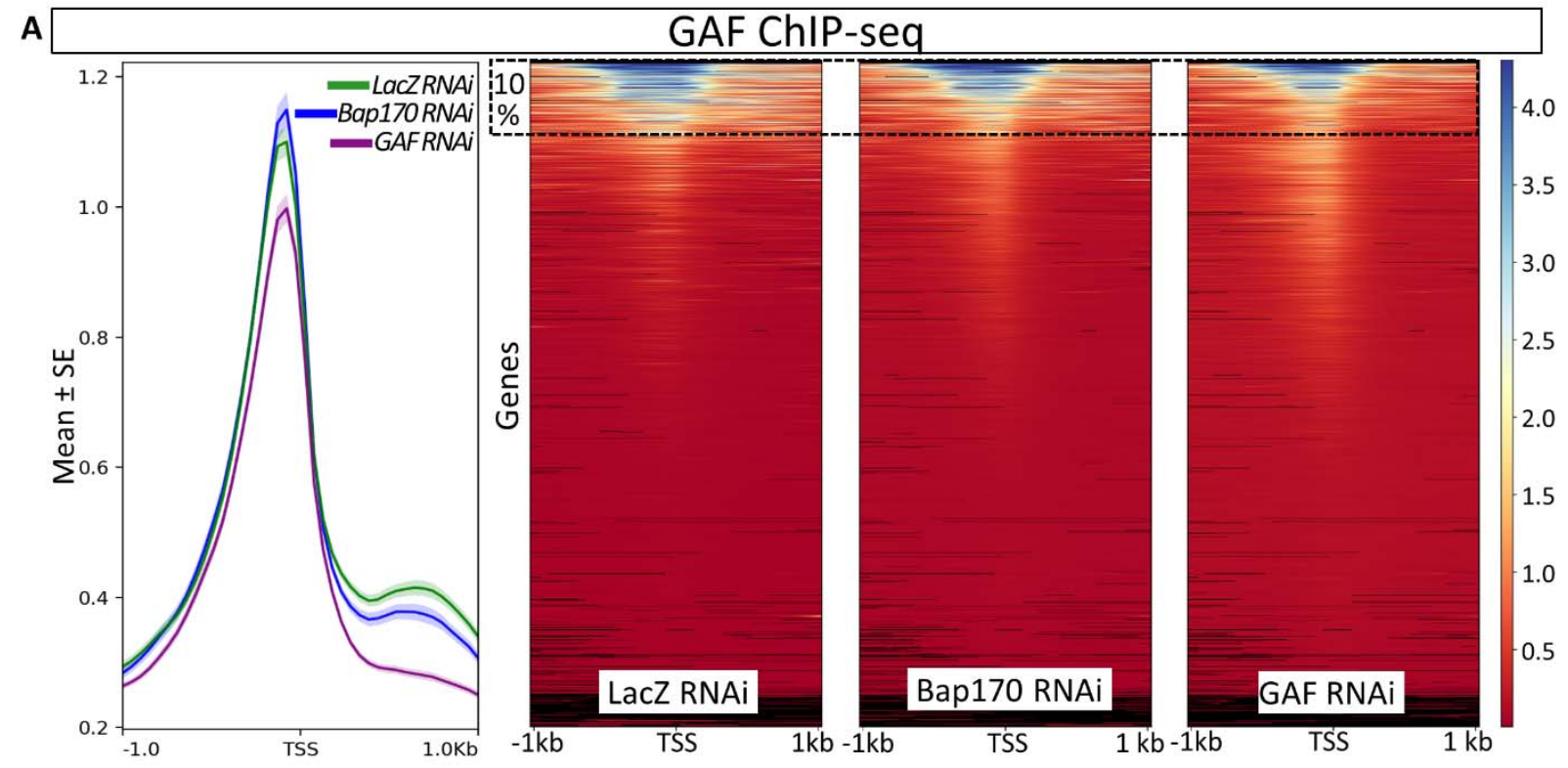

B
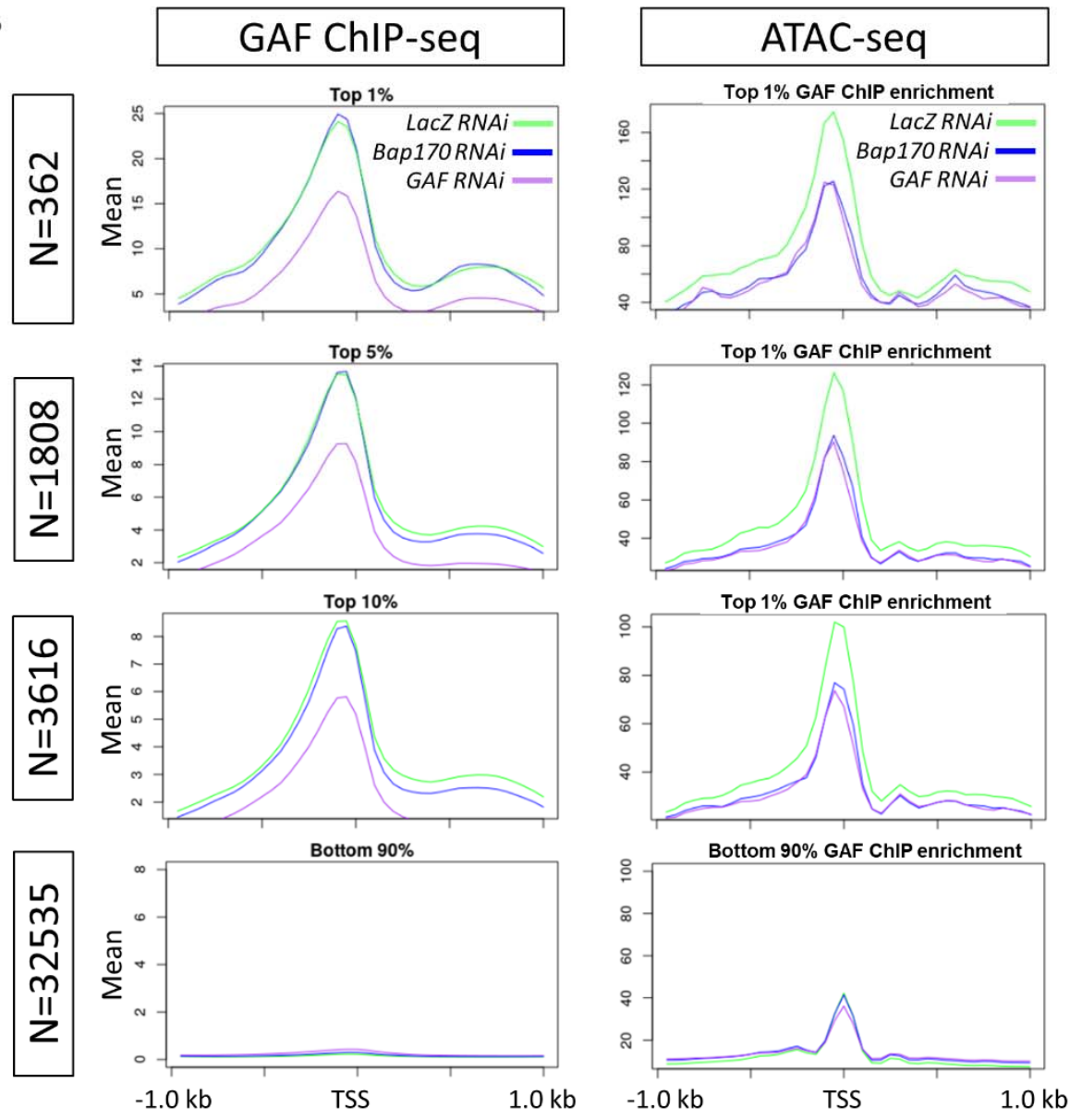
1180 Figure S7. Comparison of GAF ChIP-seq signals in LacZ RNAi (control) and Bap170 RNAi 1181 experiments of Judd et al. 2021.

(A) Comparison of GAF ChIP-seq signals in LacZ RNAi (control), Bap170 RNAi and GAF RNAi experiments (bw files from GSE157235) derived from ${ }^{24}$. The left graph shows mean ChIP enrichment (mean $\pm S E$ ) for all regions $\pm 1 \mathrm{~kb}$ centered around transcription start sites (TSS); The right graphs show heat maps of all genes (generated by computeMatrix/plotHeatmap of deepTools ${ }^{109}$ ). Dashed rectangle indicates the top $10 \%$ regions with the highest GAF ChIP enrichment in control (for which the mean enrichment is plotted in (B)).

(B) Comparison of GAF ChIP-seq, ATAC-seq, and PRO-seq signals in LacZ RNAi (control), Bap170 RNAi and GAF RNAi experiments (bw files from GSE157235, GSE149336, and GSE149332, respectively) derived from ${ }^{24}$. Regions $\pm 1 \mathrm{~kb}$ flanking TSS were sorted according to mean GAF ChIP enrichment in LacZ RNAi from high to low as shown in (A). Mean values of GAF ChIP enrichment (left column) and ATAC-seq (right column) enrichment are plotted for the top $1 \%, 5 \%, 10 \%$ of regions with the highest GAF ChIP signal and for the remaining 90\% regions. For 3616 TSS-flanking regions with highest GAF ChIP enrichment, on average, chromatin accessibilities (ATAC-seq) are reduced in both Bap170 RNAi and GAF RNAi conditions, while the mean enrichment for GAF ChIPseq shows no change in Bap170 RNAi. These analyses indicate that although there are differential effects at specific sites, the overall genome-wide GAF chromatin binding is not affected in PBAP-depleted condition. 


\section{Figure S8}

A

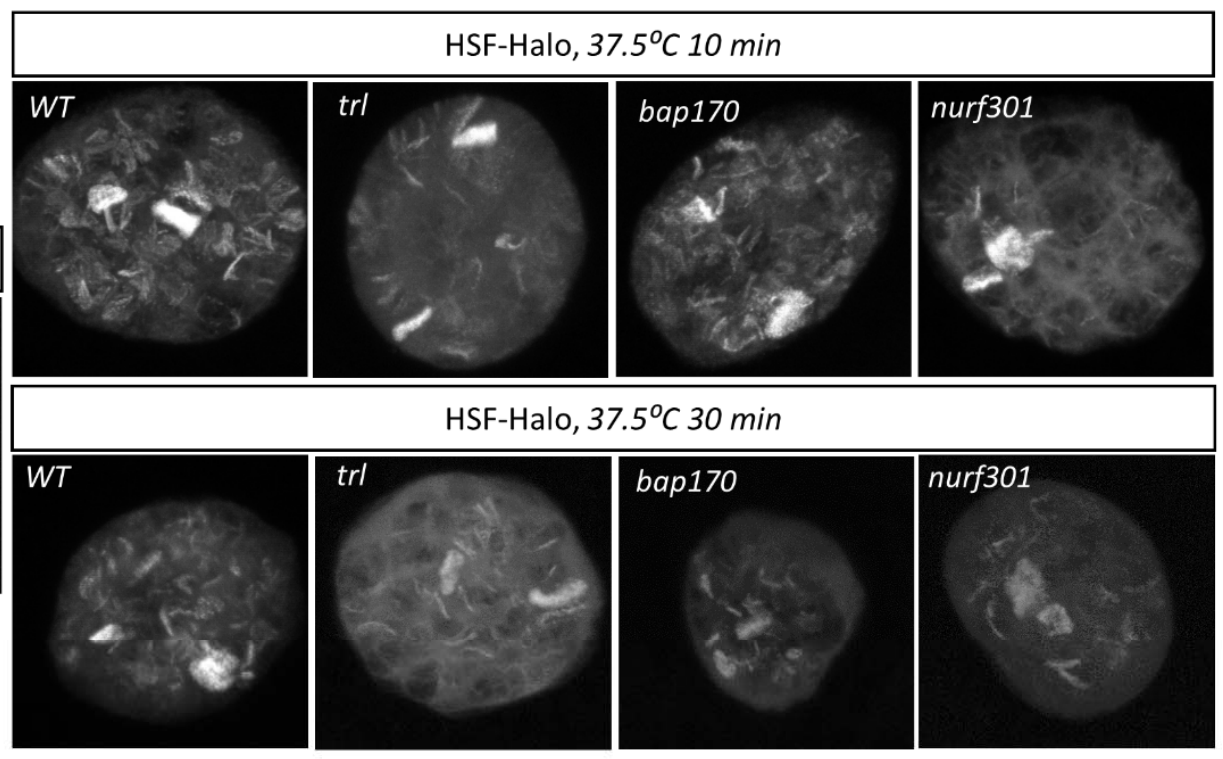

B

$10 \mathrm{~ms}$ frame rate displacement histograms and 2-state Spot-On fits
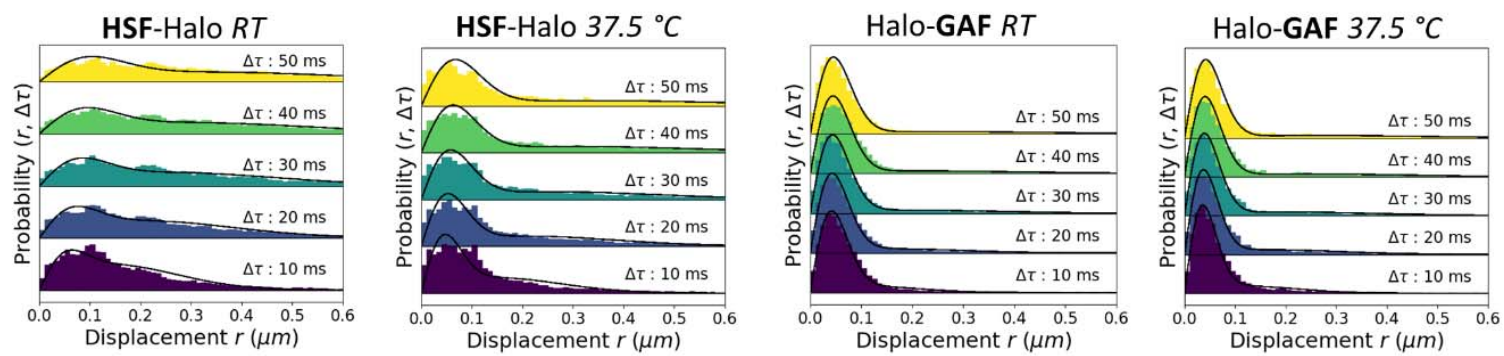

C

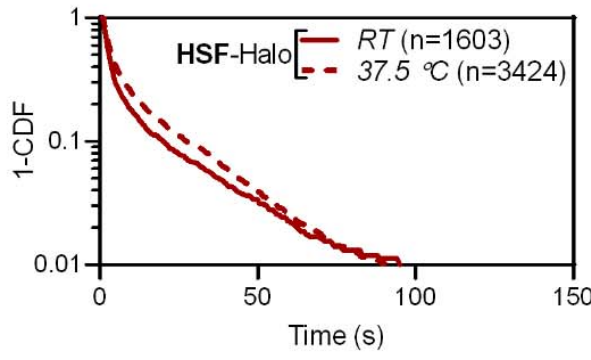

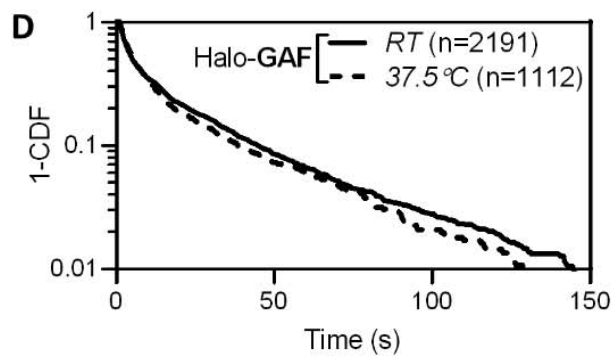

E

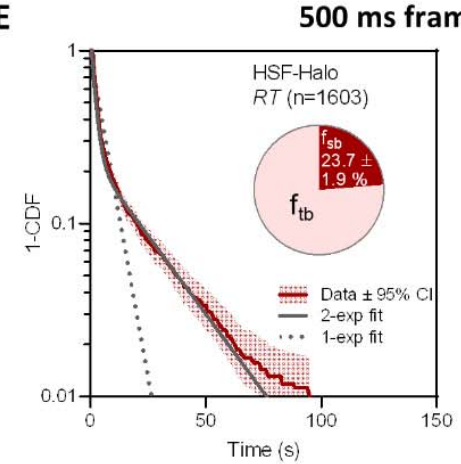

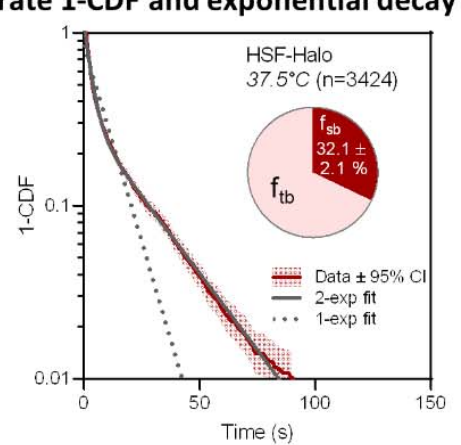

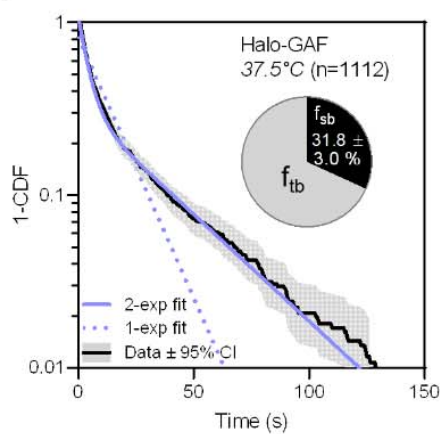


1202 Figure S8. HSF-Halo binding on polytene chromosomes and live-cell SPT at RT and HS

1203 conditions.

1204

1205

1206

1207

1208

1209

1210

1211

1212

1213

1214

1215

1216

1217

1218

1219

1220

1221

1222

(A) Confocal images of HSF-Halo in fixed salivary glands. HSF-Halo is mostly nucleoplasmic at room temperature $(R T)$ and binds to many loci after heat shock (HS) at $37.5^{\circ} \mathrm{C}$ for 10 and $30 \mathrm{~min}$. Maximum projections of confocal z-stacks are shown because major HSF bands are located in different focal planes. The pattern of HSF binding on heat shock is substantially reduced in trl and nurf301 mutants and partially affected in the bap170 mutant. Polytene loci showing little or no change of HSF binding in the trl mutant is consistent with findings that not all HS genes are GAF-dependent ${ }^{18}$. (These genes presumably require an analogous pioneer factor and attendant remodelers). See methods for genotypes of $W T, \operatorname{trl}$, bap170, and nurf 301 .

(B) Spot-On fits of fast-tracking data for HSF-Halo $\left(R T, 37.5^{\circ} \mathrm{C}\right)$ and Halo-GAF $\left(37.5^{\circ} \mathrm{C}\right.$, see Fig. $\mathrm{S} 2 \mathrm{~B}$ for $R T$ ).

(C) Survival-probability curves (1-CDF) from apparent dwell times of $>1000$ single-molecule chromatin-binding events for HSF-Halo at $R T$ and $37.5^{\circ} \mathrm{C}$.

(D) Survival-probability curves (1-CDF) from apparent dwell times of $>1000$ single-molecule chromatin-binding events for Halo-GAF at $R T$ and $37.5^{\circ} \mathrm{C}$.

(E) One-component and two-component exponential fit of survival probabilities (1-CDF) from slow tracking data for HSF-Halo $\left(R T, 37.5^{\circ} \mathrm{C}\right)$ and Halo-GAF $\left(37.5^{\circ} \mathrm{C}\right.$, see Fig. S3D for $R T)$. Pie charts show the stable-binding $\left(\mathrm{f}_{\mathrm{sb}}\right)$ and transient-binding $\left(\mathrm{f}_{\mathrm{sb}}\right)$ fractions derived from two-component fits. 


\section{Figure S9}

A vbSPT HMM analysis (2 states)
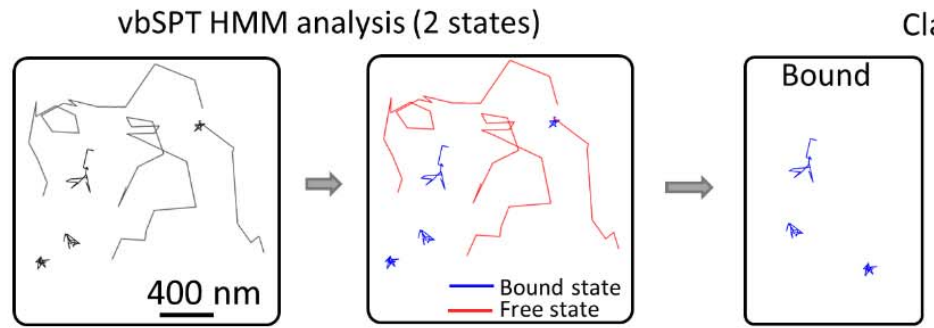

Classified trajectories

B
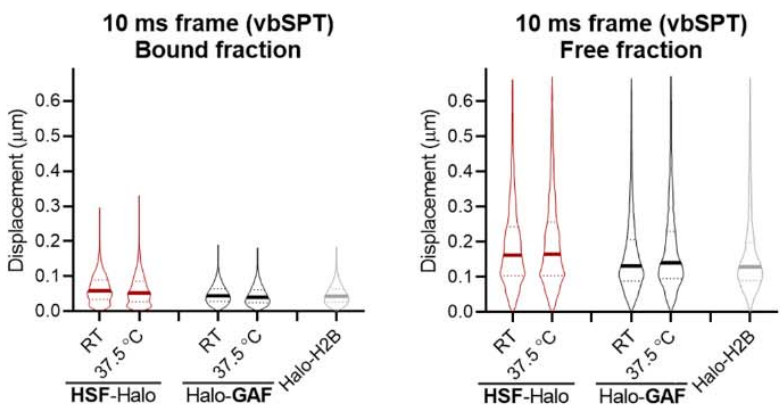

C

HSF
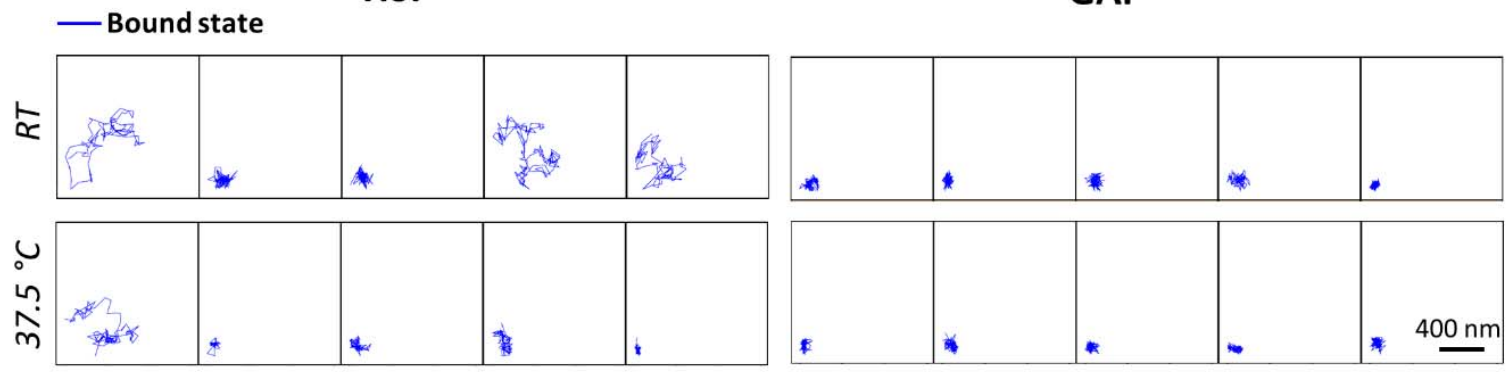

D

HSF
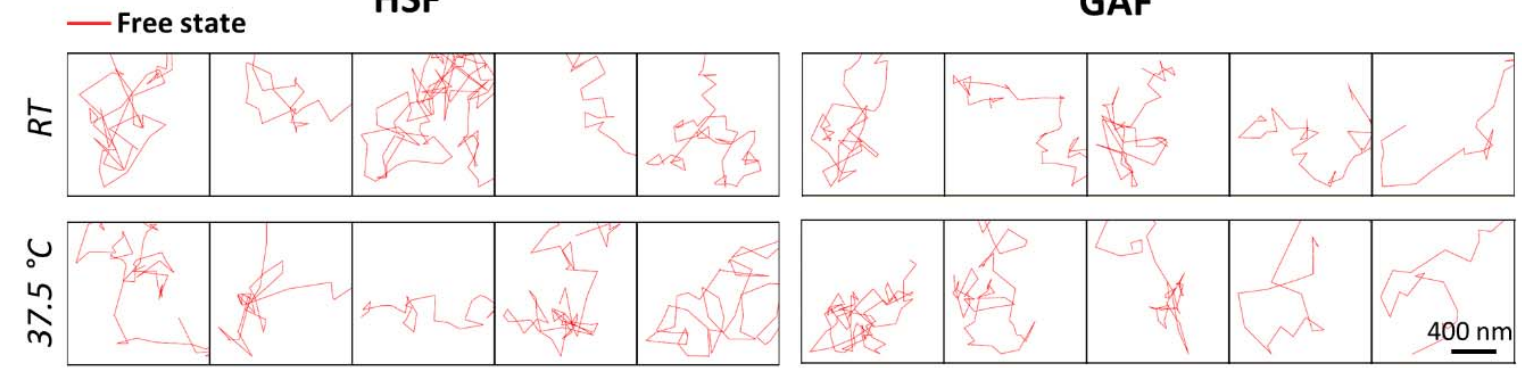

Figure S9. vbSPT analysis of fast-tracking data for HSF-Halo and Halo-GAF at RT and HS conditions.

(A) Overview of fast-tracking trajectory classification with displacement-based HMM classification (vbSPT). After assigning each displacement as either in bound or free state, each trajectory is sub-classified as 'bound' or 'free', a small fraction of trajectories containing 2 states are excluded from the following analysis in (B-G) and (Fig. 5). 
(B) Violin plots of displacements show distinct distributions for bound and free trajectories classified by vbSPT.

(C) Examples of bound trajectories classified by vbSPT for HSF-Halo, Halo-GAF at RT and $37.5^{\circ} \mathrm{C}$, and Halo-H2B at $R T$.

(D) Examples of free trajectories classified by vbSPT for HSF-Halo, Halo-GAF at $R T$ and 


\section{Figure S10}
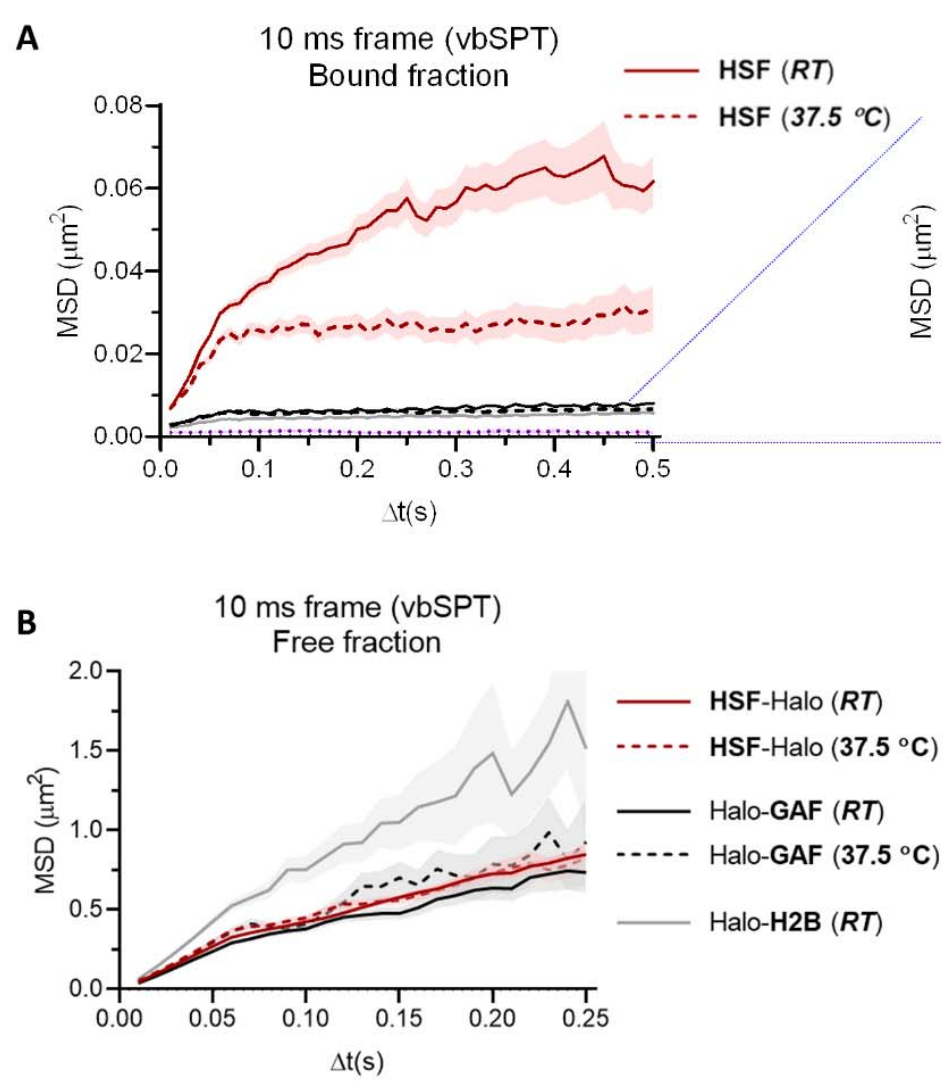

Figure S10. MSD analysis of vbSPT-classified HSF-Halo and Halo-GAF trajectories.

(A) Plot of average MSD as a function of lag time $\Delta t$ of bound trajectories classified by vbSPT for HSF-Halo, Halo-GAF at $R T$ and $37.5^{\circ} \mathrm{C}$, and Halo-H2B at $R T$. The right panel shows a zoomed-in section of the same plot. System noise is shown by MSD of dye molecules stuck on the coverglass. Mean and SE (shaded) are shown.

(B) Average MSD over $\Delta$ t of free trajectories classified by vbSPT for HSF-Halo, Halo-GAF at $R T$ and $37.5^{\circ} \mathrm{C}$, and Halo-H2B at $R T$. Mean and SE (shaded) are shown.

1244

(C) Radius of confinement (Rc) is derived by fitting individual MSD curves with a confined diffusion model, for bound trajectories classified by vbSPT, for HSF-Halo, Halo-GAF at $R T$ and $37.5^{\circ} \mathrm{C}$, and Halo- $\mathrm{H} 2 \mathrm{~B}$ at $R T$. 


\section{Figure S11}
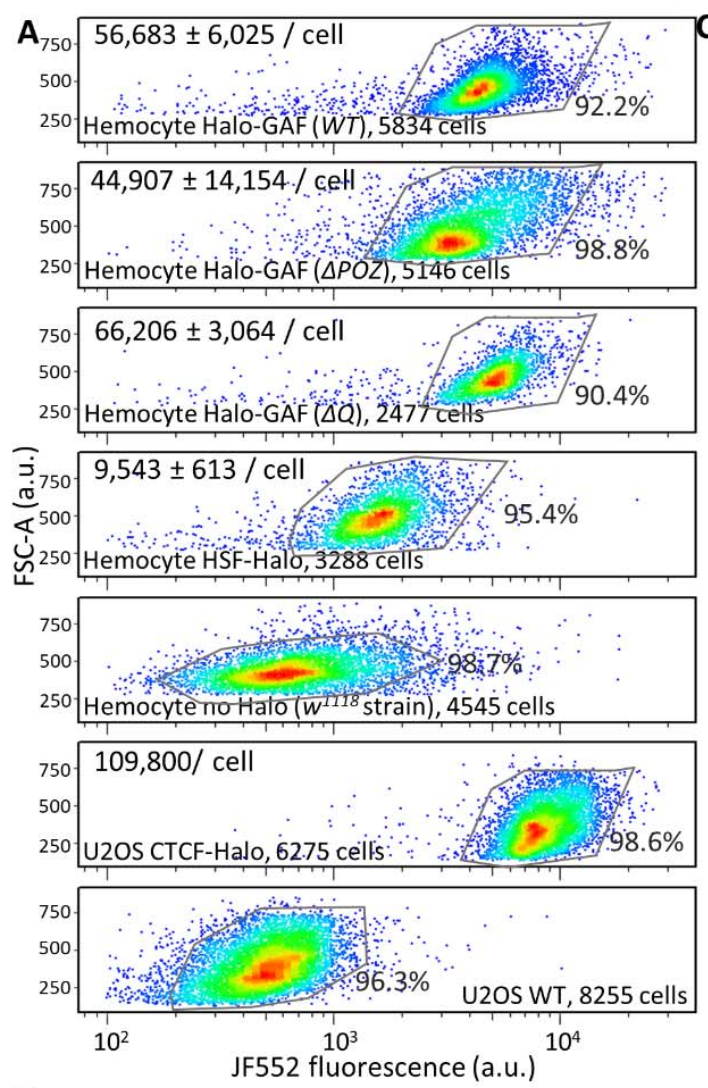

B
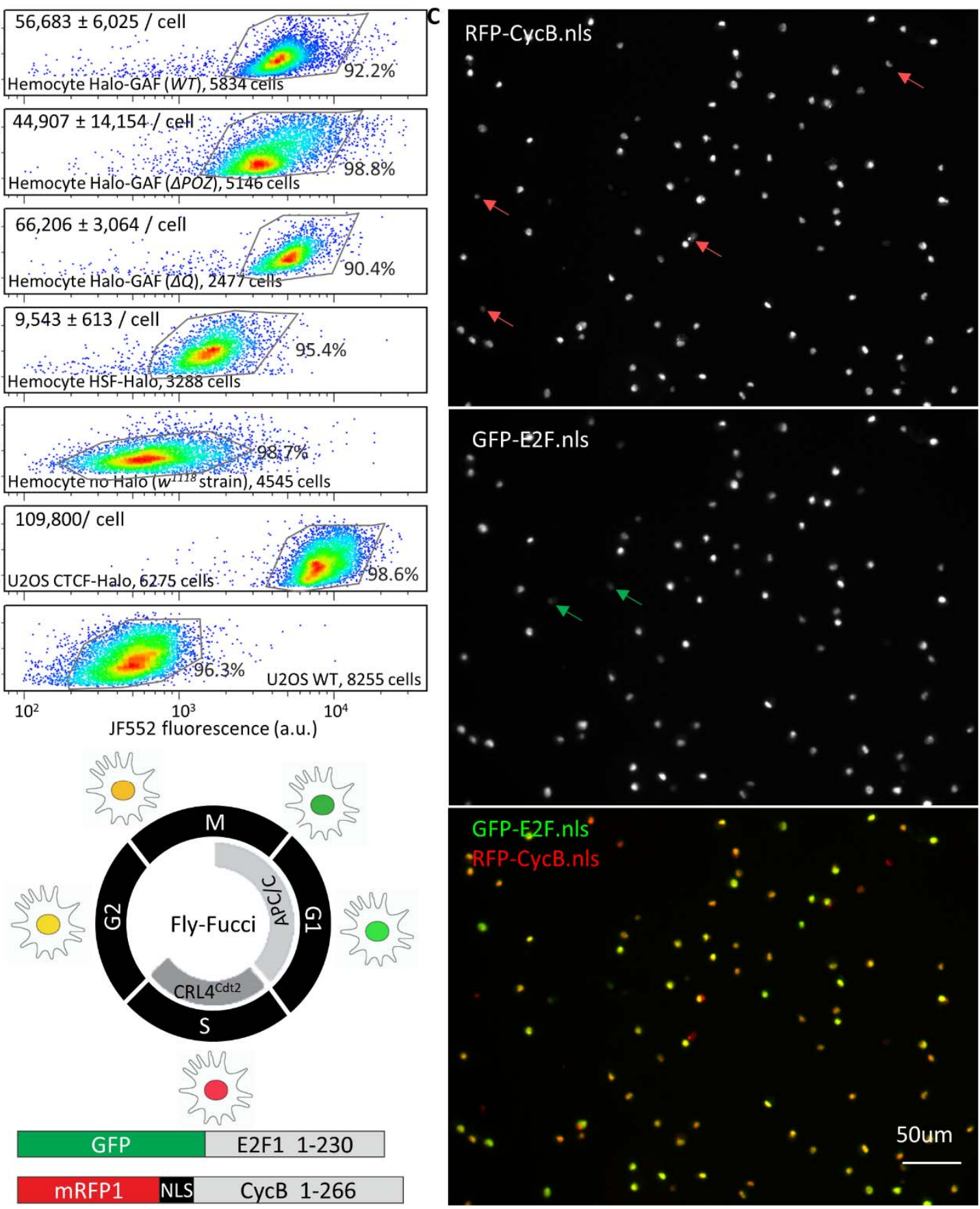

1248 Figure S11. FACS quantitation of Halo-GAF and HSF-Halo in Drosophila hemocytes and cell 1249 cycle phase identification.

(A) Total Halo-GAF (knock-in WT, $\triangle P O Z$, and $\triangle Q$ ) and HSF-Halo (transgenic in the $P\{P Z\} H s f^{03091} / H s f^{3}$ background) fluorescence per cell for JF552-stained late $3^{\text {rd }}$ instar larval hemocytes and CTCT-Halo in U2OS cells quantified by flow cytometry. Cellular 
abundance of Halo-GAF and HSF-Halo molecules are estimated using CTCF-Halo in U2OS cells as a standard (see methods) $)^{81,107}$. Hemocytes ( $w^{1118}$ strain) or U2OS cells not expressing HaloTag were used as controls for background subtraction. One of three representative flow cytometry experiments is shown. Mean \pm SD of estimated protein abundance is shown at the upper left corner of each plot. A much larger number of molecules (in the order of one million) for GAF was reported earlier in the S2 cell line ${ }^{110}$; the reason for the discrepancy is unclear. FSC-A, forward scatter area.

(B) Conceptual diagram of the Fly-FUCCI system ${ }^{111}$. Both GFP-E2F1 $1-230$ and MRFP1-CycB $_{1-266}$ are expressed with the GAL4/UAS system. In early $M$ phase, both GFP-E2F1 $1-230$ and mRFP1-CycB $B_{1-266}$ are present and thus display yellow. In mid-mitosis, the APC/C marks mRFP1-CycB $B_{1-266}$ for proteasomal degradation, leaving the cells fluorescing green. As cells progress from $\mathrm{G} 1$ to $\mathrm{S}$ phase, $\mathrm{CRL} 4{ }^{\mathrm{Cdt} 2}$ degrades GFP-E2F1 $1_{1-230}$, and cells are labeled red due to the presence of $m R F P 1-\mathrm{CycB}_{1-266}$ only. After cells enter $\mathrm{G} 2$ phase, GFP-E2F1 $1_{1-}$ 230 protein levels reaccumulate, marking the cells yellow due to the presence of mRFP1$\mathrm{CycB}_{1-266}$.

(C) Characterization of cell-cycle stage for late $3^{\text {rd }}$ instar larval hemocytes. Only 4 out of 96 cells in the field of view show 'red only' fluorescence (S phase), and 2 cells have 'green only' fluorescence ( $M$ to $\mathrm{G} 1$ phase). The majority of hemocytes have both red and green fluorescence, indicating G2 phase or early M phase. Given that a previous study shows only $0.32 \%$ of larval hemocytes stain positive with the mitotic phosH3 antibody ${ }^{112}$, we conclude that most larval hemocytes are in the G2 phase.

\section{Movie S1. Fast-tracking movie of Halo-GAF}

A fast-tracking movie of Halo-GAF labeled with 1nM JF554. Movie was acquired with $10 \mathrm{~ms}$ camera integration time for single molecule tracking after 10-30 s of initial nuclear glow.

\section{Movie S2. Slow-tracking movie of GAF - Halo}

A low-tracking movie of $\mathrm{GAF}^{\mathrm{L}}$-Halo labeled with $0.05 \mathrm{nM}$ JF552 (and $50 \mathrm{nM}$ nonfluorescent JF700 blocker). Images acquired at $500 \mathrm{~ms}$ exposure time to motion blur diffusing molecules and selectively observe chromatin-bound molecules. Movie frames are placed on a 3D-axis of time and $x$, y coordinates to display identified trajectories. Tracking parameters are adjusted to avoid identification of blurred molecules. 